\title{
SCATTERING FOR THE KLEIN-GORDON EQUATION WITH QUADRATIC AND VARIABLE COEFFICIENT CUBIC NONLINEARITIES
}

\author{
HANS LINDBLAD AND AVY SOFFER
}

\begin{abstract}
We study the 1D Klein-Gordon equation with variable coefficient cubic nonlinearity. This problem exhibits a striking resonant interaction between the spatial frequencies of the nonlinear coefficients and the temporal oscillations of the solutions. In the case where the worst of this resonant behavior is absent, we prove $L^{\infty}$ scattering as well as a certain kind of strong smoothness for the solution at time-like infinity with the help of several new normal-form transformations. Some explicit examples are also given which suggest qualitatively different behavior in the case where the strongest cubic resonances are present.
\end{abstract}

\section{INTRODUCTION}

In this paper we study the asymptotic behavior of solutions to nonlinear KleinGordon (KG) equations in one space dimension, with variable coefficients depending on the point in space. These kinds of equations show up in the stability analysis of stationary solutions to some equations of mathematical physics.

The most important one dimensional Klein-Gordon type equations are the well known $\phi^{4}$ sigma model:

$$
\partial_{t}^{2} \phi-\partial_{x}^{2} \phi-\phi+\phi^{3}=0
$$

which has the stationary 'kink' solution $\phi_{0}=\tanh (x)$, and the sine-Gordon equation:

$$
\partial_{t}^{2} \phi-\partial_{x}^{2} \phi+\sin \phi=0
$$

which has the stationary solution $\phi_{0}=\arctan \left(e^{x}\right)$. In both cases the substitution $\phi=\phi_{0}+u$ gives a nonlinear Klein-Gordon equation for $u$ with coefficients depending on the point in space.

Topological solitons, which are static solutions of nonlinear equations of mathematical physics, play an important role in many models of field theory, statistical models and nonlinear dynamics $[\mathrm{M}-\mathrm{S}]$.

Topological solitons differ from the standard soliton solutions by being topologically inequivalent to the vacuum solution or the zero/constant solution. As such, they are usually characterized by a topological "charge", a winding number associated to the solution. In one dimension such solutions are the kink solutions, characterized by having a different constant limit as $x$ approaches + or - infinity.

Received by the editors August 13, 2013 and, in revised form, April 6, 2014.

2010 Mathematics Subject Classification. Primary 35Qxx.

Key words and phrases. Nonlinear KG equation, long-range scattering, normal form analysis.

The first author was partially supported by NSF grant DMS-1237212.

The second author was partially supported by NSF grant DMS-1201394. 
In two dimensions topological solitons are vortices, for example, in three dimension monopoles and in four dimension instantons.

An important aspect of the analysis of such solutions is the dynamics of a perturbed kink. Since, by topological restriction, they cannot approach a vacuum state, they are stable. But, they can radiate some field and change their parameters, such as velocity, in the process.

Under small perturbation, one is led to consider the solution as a sum of kinks plus a remainder:

$$
\phi=K(x, \gamma(t))+u(x, t),
$$

where, to determine the equations of the parameters of the kink, $\gamma(t)$, as a function of time, one needs to impose further orthogonality conditions on $u$.

The resulting equation for $u$ is easily seen to be a nonlinear Klein-Gordon equation which also depends on $\dot{\gamma}(t)$.

The nonlinear terms, in general, will include terms which are linear, quadratic and cubic, with powers of $K$ as coefficients.

In one dimension, the quadratic and cubic terms are leading to serious long range problems. It is well known that in general, they modify the large time behavior of the solution, and it is not given by the simple free Klein-Gordon waves Str, Sh, D1. The problem also shows up in the Schrödinger equation; see e.g. $[\mathrm{H}-\mathrm{Nau}$. To understand the problem, notice that in one dimension the solution of the free KG equation decays, in the $L^{\infty}$ norm, like $t^{-1 / 2}$. Hence the quadratic and cubic terms act like potentials that decay like $t^{-1 / 2}$ and $t^{-1}$, respectively. These are not integrable at infinity, and therefore lead to modified free dynamics.

The way to deal with these terms is based on normal-form transformations, which is an effective way of using the oscillation in the solution to integrate by parts and gain extra decay.

This was first done by Shatah [Sh] for nonlinear partial differential equations. See also $\mathrm{F}$, Kat. The case of Klein-Gordon equations with quadratic and cubic terms was treated by Delort D1. A different approach was later developed by LindbladSoffer L-S1, L-S2, which gave a simplified and detailed asymptotic expansion of the solution for large times, for both the nonlinear KG and NLS, with cubic terms.

All of the above works assume constant coefficient nonlinear (and linear) terms. The work we present here is dealing with a nonconstant coefficient cubic term and a constant quadratic term. It turns out that there is a fundamental new resonance phenomena in this case between the oscillation in $x$ space of the coefficient function, denoted below by $\beta(x)$, and the nonlinearity! These resonances may have different manifestations, and they change the behavior of the solutions. The treatment for such resonances requires pushing the method of normal forms in a new direction.

In this work we then consider the large time Cauchy problem for the one dimensional Klein-Gordon equation of the following type:

$$
\begin{aligned}
\partial_{t}^{2} u-\partial_{x}^{2} u+u & =\alpha_{0} u^{2}+\left(\beta_{0}+\beta(x)\right) u^{3}, \\
u(0, x) & =u_{0}(x), \\
\partial_{t} u(0, x) & =u_{1}(x),
\end{aligned}
$$

for smooth compactly supported initial data $\left(u_{0}, \dot{u}_{0}\right)$. Here we assume that the function $\beta(x)$ is a rapidly decaying smooth function in $x$. 
Theorem 1.1. Let $u(t, x)$ be the global solution to the system (1.1) with sufficiently small compactly supported initial data $\left(u_{0}, \dot{u}_{0}\right)$ in the Sobolev norm $\left(H^{2}, H^{1}\right)$. Then for $t \geqslant 0$ the solution $u(t, x)$ obeys the following $L^{\infty}$ estimate:

$$
|u(t, x)| \lesssim \frac{C\left(u_{0}, u_{1}\right)}{(1+|\rho|)^{\frac{1}{2}}}, \quad \rho=\left|t^{2}-x^{2}\right|^{1 / 2} .
$$

We remark that even in the constant coefficient case, when $\beta(x)=\beta_{0}$ is constant, the asymptotic behavior is nontrivial. Delort proved that there is a logarithmic phase correction to the asymptotics of the free linear Klein-Gordon:

$$
v(t, x) \sim \rho^{-1 / 2} e^{i \phi_{0}(\rho, x / \rho)} a(x / \rho)+\rho^{-1 / 2} e^{-i \phi_{0}(\rho, x / \rho)} \overline{a(x / \rho)},
$$

were $a(x / \rho)=\sqrt{1+x^{2} / \rho^{2}} \widehat{u}_{+}(-x / \rho)$ is obtained from the Fourier transform of initial data $\widehat{u}_{+}(\xi)=\left(\widehat{u}_{0}(\xi)-i\left(|\xi|^{2}+1\right)^{-1 / 2} \widehat{u}_{1}(\xi)\right) / 2$ and the phase is given by

$$
\phi_{0}(\rho, x / \rho)=\rho+\left(\frac{3}{8} \beta_{0}+\frac{5}{12} \alpha_{0}^{2}\right)|a(x / \rho)|^{2} \ln \rho .
$$

Remark 1.2. It should be noted that we only get decay in powers of $\rho$, but not $t$. Interestingly, it shows that this weaker type of decay is, in fact, sufficient for us to prove global existence. On the other hand, to recover from our results the free decay estimate, $t^{-1 / 2}$ for the $L^{\infty}$ norm, more analysis is required. One has to estimate the invariant Klainerman-Sobolev norms, adapted to the one dimensional $\mathrm{KG}$ equation. In our case we can only use derivatives of order two. But we do have control of the scaling and boost to order two in $L^{2}$.

This issue and further applications of the analysis in this paper, that is, the asymptotic scattering and phase behavior, will be considered elsewhere.

To proceed, we first change coordinates to hyperbolic:

$$
x=\rho \sinh (y), \quad t=\rho \cosh (y) .
$$

Then, we extract the leading order behavior at large time $\rho$ :

$$
v=\rho^{\frac{1}{2}} u \text {. }
$$

Then the equation is

$$
\left(\partial_{\rho}^{2}-\frac{1}{\rho^{2}} \partial_{y}^{2}+1+\frac{1}{4 \rho^{2}}\right) v=\frac{\alpha_{0}}{\rho^{\frac{1}{2}}} v^{2}+\frac{\beta_{0}}{\rho} v^{3}+\frac{\beta(\rho y)}{\rho} v^{3}+\mathcal{R},
$$

with a small remainder

$$
\mathcal{R}=\mathcal{R}_{\beta} v^{3}, \quad \text { where } \quad \mathcal{R}_{\beta}(\rho, y)=\beta(\rho \sinh (y))-\beta(\rho y) .
$$

Most of the effort in our proof of the decay estimate (1.2) centers on obtaining simple pointwise in time Sobolev bounds for the function $v(\rho, y)$ in the region where $|y| \lesssim 1$. The main difficulty in closing such estimates stems from the fact that the quadratic term does not have even close to integrable decay, and also the fact that the coefficient function $\beta$ on the RHS of (1.6) is extremely rough in the $y$ variable for large values of $\rho$. Indeed, one can see that unless this second factor is constant, the cubic nonlinearity has no pointwise decay in $\rho$ once a $\partial_{y}$ derivative is applied to it, and it is hopeless to try to apply the energy estimate to expressions of this form. However, with the help of our rather involved normal-form transformations applied to the solution $v$, we will in fact be able to subtract off the leading behavior and show that one may recover the same bounds one would get by simply differentiating the equation and then applying the energy estimate. 
In the usual approach, one introduces a near identity nonlinear change of variables of the function $v$ :

$$
v=w_{0}+w_{1}
$$

and

$$
w_{1}=\frac{1}{\rho^{\frac{1}{2}}} B_{1}(v, v)+\frac{1}{\rho^{\frac{1}{2}}} B_{2}(\dot{v}, \dot{v}),
$$

where the operators are bilinear $\Psi \mathrm{DO}$ as defined on line (5.4). Here we are using the shorthand $\partial_{\rho} u=\dot{u}$, and similarly for $\dot{v}$. Here $w_{0}$ solves the free Klein-Gordon equation, i.e. (1.6) but with vanishing right hand side. Using this normal-form transformation gets rid of the quadric term in the right hand side and produces a nonlocal cubic term in its places. Apart from the nonlocal cubic term, which pretty much can be dealt with as the local cubic term $\beta_{0} \neq 0$, we have reduced to the case $\alpha_{0}=0$ in the above. Since the term with $\rho^{-2} v$ in the left of (1.6) clearly decays much more, we will for simplicity now neglect it and consider only the linear operator

$$
\square_{\mathcal{H}}+1, \quad \text { where } \quad \square_{\mathcal{H}}=\partial_{\rho}^{2}-\frac{1}{\rho^{2}} \partial_{y}^{2} .
$$

1.1. A simplified model describing the problem with variable coefficients. The case of constant coefficient cubic $\beta_{0} \neq 0$ but $\beta=0$ and $\alpha_{0}=0$ is by now much easier to deal with as in L-S2. We will roughly describe the argument there and an important modification that allows us to deal with quintic variable coefficients:

$$
\left(\partial_{\rho}^{2}-\frac{1}{\rho^{2}} \partial_{y}^{2}+1\right) v=F, \quad \text { where } \quad F=\frac{\beta_{0}}{\rho} v^{3}+\frac{\beta(\rho y)}{\rho^{3 / 2}} v^{4} .
$$

The argument below will involve bounds for the $H^{1}$ norm as well as the $L^{\infty}$ norm and will be the same norms we will bound for the general case. The argument will also show why we can't directly deal with the variable coefficient cubic.

First we have the energy estimate (obtained by multiplying (1.10) by $\dot{v}$ and integrating)

$$
\left\|\left(v, \dot{v}, \rho^{-1} \partial_{y} v\right)(\rho)\right\|_{H^{1}} \lesssim\left\|\left(v, \dot{v}, \rho^{-1} \partial_{y} v\right)(1)\right\|_{H^{1}}+\int_{1}^{\rho}\|F(s)\|_{H^{1}} d s,
$$

where $F$ is the right hand side of (1.10):

$$
\begin{aligned}
\|F(\rho)\|_{H^{1}} \lesssim \frac{1}{\rho}\|v(\rho)\|_{L^{\infty}}^{2}\|v(\rho)\|_{H^{1}} & +\frac{1}{\rho^{3 / 2}}\left\|\rho \beta^{\prime}(\rho y)\right\|_{L_{y}^{2}}\|v(\rho)\|_{L^{\infty}}^{4} \\
& \lesssim \frac{1}{\rho}\left(1+\|v(\rho)\|_{L^{\infty}}\right)^{2}\|v(\rho)\|_{L^{\infty}}^{2}\|v(\rho)\|_{H^{1}}
\end{aligned}
$$

since $\left\|\rho \beta^{\prime}(\rho y)\right\|_{L_{y}^{2}}=\rho^{1 / 2}\left\|\beta^{\prime}\right\|_{L^{2}}$. We want to prove that for sufficiently small initial data $\left\|\left(v, \dot{v}, \rho^{-1} \partial_{y} v\right)(1)\right\|_{H^{1}} \leq \epsilon$ we have a global bound

$$
\|v(\rho)\|_{L^{\infty}} \leq C \epsilon .
$$

The strategy is to assume that we have this bound and prove that it implies a better bound. It then follows that

$$
\left\|\left(v, \dot{v}, \rho^{-1} \partial_{y} v\right)(\rho)\right\|_{H^{1}} \lesssim\left\|\left(v, \dot{v}, \rho^{-1} \partial_{y} v\right)(1)\right\|_{H^{1}}+C \int_{1}^{\rho} \frac{\epsilon^{2}}{s}\left\|\left(v, \dot{v}, \rho^{-1} \partial_{y} v\right)(s)\right\|_{H^{1}} d s,
$$


which gives a bound

$$
\left\|\left(v, \dot{v}, \rho^{-1} \partial_{\rho} v\right)(\rho)\right\|_{H^{1}} \leq C \epsilon \rho^{C \epsilon} .
$$

We now also need to recover the $L^{\infty}$ bound we just used. Sobolev's lemma (1.15) gives a weaker decay estimate (1.13) but with a growing factor $\rho^{C \epsilon}$ which is not sufficient. If, however, the variable coefficient term $\beta$ is not present, one can differentiate the equation further and obtain bounds of the form (1.15) also for the $H^{k}$ norms, and hence Sobolev's lemma gives weaker decay estimates also for higher derivatives, e.g. $\left\|\partial_{y}^{2} v(\rho)\right\|_{L_{y}^{\infty}} \leq\|v(\rho)\|_{H^{3}}$. This was used in [L-S2] together with additional estimates obtained by moving the term $\rho^{-1} \partial_{y}^{2} v$ to the right hand side and integrating the left hand side of the equation as an ODE (i.e. multiplying by $\dot{v}$ first):

$$
\left(\partial_{\rho}^{2}+1\right) v-\frac{\beta_{0}}{\rho} v^{3}=\frac{1}{\rho^{2}} \partial_{y}^{2} v+\frac{\beta(\rho y)}{\rho^{3 / 2}} v^{4},
$$

which gives the estimate

$$
\|(v, \dot{v})(\rho)\|_{L^{\infty}} \lesssim\|(v, \dot{v})(1)\|_{L^{\infty}}+\int_{1}^{\rho}\|G(s)\|_{L^{\infty}} d s,
$$

where $G$ is the right hand side of (1.16):

$$
\|G(\rho)\|_{L^{\infty}} \lesssim \frac{1}{\rho^{2}}\left\|\partial_{y}^{2} v(\rho)\right\|_{L^{\infty}}+\frac{1}{\rho^{3 / 2}}\|v(\rho)\|_{L^{\infty}}^{4}
$$

is integrable if $\left\|\partial_{y}^{2} v(\rho)\right\|_{L^{\infty}} \lesssim \epsilon \rho^{a}$ for some $a<1$. This would recover (1.13). (We remark that similar ideas were also used in [L1], [L-R] for the wave equation and is related to the weak null condition.) However, because of the presence of the variable coefficient term $\beta(\rho y)$ we cannot differentiate more than once with respect to $y$, since powers of $\rho$ come out, so there is no hope of $H^{k}$ bounds for $k>1$.

The new idea here is to instead introduce a frequency projection onto low and high frequencies $I=P_{\leq \rho^{\sigma}}+P_{\geq \rho^{\sigma}}$. Let $v_{1}=P_{\leq \rho^{\sigma}} v$ and $v_{2}=P_{\geq \rho^{\sigma}} v$. Since $\left\|P_{\geq \lambda} v\right\|_{L^{\infty}}^{2} \leq\left\|P_{\geq \lambda} v\right\|_{L^{2}}\left\|P_{\geq \lambda} v\right\|_{H^{1}} \leq \lambda^{-1 / 2}\|v\|_{H^{1}}^{2}$ it follows that

$$
\left\|P_{\geq \rho^{\sigma}} v\right\|_{L^{\infty}} \lesssim \rho^{-\sigma / 2}\|v(\rho)\|_{H^{1}} \lesssim \epsilon \rho^{C \epsilon-\sigma / 2} \lesssim \epsilon .
$$

It only remains to bound the low frequencies and for this we use a version of the ODE argument above. The bound for the low frequency part then follows from projecting the equation (1.16) to low frequencies:

$$
\left(\partial_{\rho}^{2}+1\right) v_{1}-\frac{\beta_{0}}{\rho} v_{1}^{3}=\frac{1}{\rho^{2}} \partial_{y}^{2} v_{1}+\frac{\beta_{0}}{\rho}\left(v^{3}-v_{1}^{3}\right)+\frac{\beta(\rho y)}{\rho^{3 / 2}} v^{4}-P_{\geq \rho^{\sigma}} F,
$$

where $F$ is as in (1.10). By a similar argument used to prove (1.19) we have

$$
\left\|\partial_{y}^{2} v_{1}(\rho)\right\|_{L^{\infty}} \leq C \rho^{3 \sigma / 2}\|v(\rho)\|_{H^{1}} \leq C \epsilon \rho^{3 \sigma / 2+C \epsilon}
$$

and

$$
\left\|v^{3}-v_{1}^{3}\right\|_{L^{\infty}} \lesssim\left\|v_{2}\right\|_{L^{\infty}}\left(\left\|v_{1}\right\|_{L^{\infty}}+\left\|v_{2}\right\|_{L^{\infty}}\right)^{2} \lesssim \frac{1}{\rho^{\sigma / 2}}\|v\|_{H^{1}}\|v\|_{L^{\infty}}^{2}
$$

since the kernels of the projections are uniformly bounded in $L^{1}$, and

$$
\left\|P_{\geq \rho^{\sigma}} F\right\|_{L^{\infty}} \leq \rho^{-\sigma / 2}\|F\|_{H^{1}},
$$

which is bounded by (1.12). The $L^{\infty}$ norm of the right hand side (1.20) is again integrable if $C \epsilon<\sigma<2 / 3-C \epsilon$. 
1.2. Variable coefficient cubic normal-form transformations. For the variable coefficient cubic term $\beta \neq 0$, we have seen that the above argument doesn't work directly, but we need to first remove it with a new type of variable coefficient normal-form construction, given in section 7 . To simply let us just deal with the case

$$
\left(\partial_{\rho}^{2}-\frac{1}{\rho^{2}} \partial_{y}^{2}+1\right) v=\frac{\beta(\rho y)}{\rho} v^{3}
$$

but using the same kind of norms as in the previous example. First we note:

The decay estimate in the previous example works just as before: one just replaces $\beta_{0}$ with $\beta$ in the left of (1.16) and estimate it as an ODE by multiplying by $\dot{v}$ and integrating the terms in the left modulo errors that can be estimated; see section 4. Hence it is only the energy estimate that involves taking one $y$ derivative of the right hand side of (1.24) in $L^{2}$ that fails, and it is only when that derivative falls on $\beta(\rho y)$. Moreover it is only the frequencies of $v$ less than $\leq \rho$ that cause problems since $\|\beta(\rho y)\|_{H_{y}^{1}} \sim \rho^{1 / 2}$ and $\left\|P_{\geq \rho} v\right\|_{L^{\infty}} \leq C \rho^{-1 / 2}\|v\|_{H^{1}}$.

We will therefore attempt to find and subtract off a normal form $w_{2}$ such that

$$
\left(\partial_{\rho}^{2}-\frac{1}{\rho^{2}} \partial_{y}^{2}+1\right) w_{2}=\frac{\beta(\rho y)}{\rho}\left(P_{\leq \rho} v\right)^{3}+\mathcal{E}_{\text {cubic }}
$$

modulo an error

$$
\left\|\mathcal{E}_{c u b i c}\right\|_{H^{1}} \lesssim \frac{1}{\rho}\|(v, \dot{v})\|_{L^{\infty}}^{2}\|(v, \dot{v})\|_{H^{1}}
$$

that can be absorbed in the energy estimate as described in the previous section; see (1.11)-(1.15).

We will attempt a new type of normal-form transformation:

$$
w_{2}=\frac{1}{\rho} \sum_{i=0}^{3} f_{i} F_{i}\left(v_{0}, \dot{v}_{0}\right), \quad \text { where } \quad F_{i}(v, \dot{v})=v^{3-i} \dot{v}^{i}, \quad v_{0}=P_{\leq \rho} v,
$$

where we assume that we can find some functionals $f_{i}$ of $\beta$ :

$$
f_{i}=f_{i}[\beta]
$$

behaving like $\beta(\rho y)$ :

$$
\left\|\partial_{\rho}^{\ell} D_{y}^{k} f_{i}\right\|_{L_{y}^{\infty}} \leq C, \quad\left\|\partial_{\rho}^{\ell} D_{y}^{k} f_{i}\right\|_{H^{1}} \leq C \rho^{1 / 2}, \quad \text { for } \quad k, \ell \leq 2
$$

and satisfying some equations to be determined such that 1.25 hold. It will be easy to see from this and the arguments below that

$$
\left\|\left(w_{2}, \dot{w}_{2}, D_{y} w_{2}\right)\right\|_{H^{1}} \lesssim \rho^{-1 / 2}\left\|\left(v, \dot{v}, D_{y} v\right)\right\|_{L^{\infty}}^{2}\left\|\left(v, \dot{v}, D_{y} v\right)\right\|_{H^{1}} .
$$

Note that the operator

$$
D_{y}=\frac{1}{i \rho} \partial_{y}
$$

is a bounded operator on $P_{\leq \rho} v$ in $L^{2}$ and anyway it is also part of the energy estimate. It follows that as long as (1.29) holds, any term with at least one $D_{y}$ derivative falling on $F_{i}$ or $\left(v_{0}, \dot{v}_{0}\right)$ is easy to control, since if $k \geq 1$, then

$$
\left\|D_{y}^{k}\left(v_{0}, \dot{v}_{0}\right)\right\|_{H^{1}} \lesssim \rho^{-1}\|(v, \dot{v})\|_{H^{1}}, \quad\left\|D_{y}^{k}\left(v_{0}, \dot{v}_{0}\right)\right\|_{L^{\infty}} \lesssim \rho^{-1 / 2}\|(v, \dot{v})\|_{H^{1}} .
$$


Hence only $\rho$ derivatives falling on $F_{i}$ matter. Also any $\rho$ derivative falling on $\rho^{-1}$ produces a term with additional decay and hence easy to control. We therefore have

$$
\left(\square_{\mathcal{H}}+1\right) w_{2} \sim \frac{1}{\rho} \sum_{i=0}^{3}\left[\left(\square_{\mathcal{H}}+1\right) f_{i}\right] F_{i}+f_{i} \partial_{\rho}^{2} F_{i}+2 \partial_{\rho} f_{i} \partial_{\rho} F_{i},
$$

modulo terms that decay faster or are bounded in $H^{1}$ and satisfy $(1.26)$.

Using the equation

$$
\ddot{v}_{0}+v_{0}=D_{y}^{2} v_{0}+\frac{1}{\rho} P_{\leq \rho}\left(\beta v^{3}\right)+\left[\partial_{\rho}^{2}, P_{\leq \rho}\right] v \sim 0
$$

to replace $\ddot{v}_{0}$ by $-v_{0}$, we obtain polynomials of degree $3, F_{i}^{0}=F_{i}$ and

$$
F_{i}^{1}(v, \dot{v})=\left.\left(\partial_{\rho} F_{i}\right)(v, \dot{v}, \ddot{v})\right|_{\ddot{v}=-v}, \quad F_{i}^{2}(v, \dot{v})=\left.\left(\partial_{\rho}^{2} F_{i}\right)(v, \dot{v}, \ddot{v}, \dddot{v})\right|_{\ddot{v}=-v, \dddot{v}=-\dot{v}}
$$

where

$$
\begin{aligned}
& F_{i}^{1}=(3-i) F_{i+1}-i F_{i-1}, \\
& F_{i}^{2}=(3-i)(2-i) F_{i+2}+i(i-1) F_{i-2}-((3-i)(i+1)+(4-i) i) F_{i} .
\end{aligned}
$$

The errors in these approximations are

$$
\begin{aligned}
& \partial_{\rho} F_{i}-F_{i}^{1}=G_{i}^{1}\left(\ddot{v}_{0}+v_{0}\right), \\
& \partial_{\rho}^{2} F_{i}-F_{i}^{2}=G_{i}^{2}\left(\ddot{v}_{0}+v_{0}\right)+G_{i}^{3}\left(\ddot{v}_{0}+v_{0}\right)^{2}+G_{i}^{4} \partial_{\rho}\left(\ddot{v}_{0}+v_{0}\right),
\end{aligned}
$$

where $G_{i}^{k}=G_{i}^{k}\left(v_{0}, \dot{v}_{0}\right)$ are polynomials such that all terms are cubic and $\ddot{v}_{0}+v_{0}$ is given by (1.34). The first term in the right of (1.34) is easy to control using (1.32). The other two terms have more than enough additional decay since

$$
\begin{aligned}
\left\|\left[\partial_{\rho}, P_{\leq \rho}\right] u\right\|_{L^{\infty}} & \lesssim(1 / \rho)\left\|P_{\sim \rho} u\right\|_{L^{\infty}} \leq(1 / \rho) \rho^{-1 / 2}\|u\|_{H^{1}}, \\
\left\|\left[\partial_{\rho}, P_{\leq \rho}\right] u\right\|_{H^{1}} & \lesssim(1 / \rho)\|u\|_{H^{1}} .
\end{aligned}
$$

With this in mind we want to solve the system

$$
\sum_{i=0}^{3}\left[\left(\square_{\mathcal{H}}+1\right) f_{i}\right] F_{i}+f_{i} F_{i}^{2}+2 \partial_{\rho} f_{i} F_{i}^{1} \sim \beta(\rho y) F_{0}
$$

by equating the coefficients of the monomials $F_{i}$, at least up to terms decaying faster.

Our first attempt is to assume that

$$
f_{i}=f_{i}(\rho y), \quad \text { where } \quad\left|f_{i}^{(k)}(z)\right| \leq C(1+|z|)^{-k}, \quad k \leq 2,
$$

in which case (1.29) holds and in addition $\left|\partial_{\rho}^{k} f_{i}\right| \leq C \rho^{-k}$ so that we also can neglect the terms with $\rho$ derivatives falling on $f_{i}$. Then the above system simplifies to

$$
\sum_{i=0}^{3}\left[\left(f_{i}(\rho y)-f_{i}^{\prime \prime}(\rho y)\right] F_{i}+f_{i}(\rho y) F_{i}^{2}=\beta(\rho y) F_{0} .\right.
$$

This simplifies to $f_{1}=f_{3}=0$ and

$$
\begin{aligned}
& f_{0}^{\prime \prime}(z)+2 f_{0}(z)-2 f_{2}(z)=-\beta(z), \\
& f_{2}^{\prime \prime}(z)+6 f_{2}(z)-6 f_{0}(z)=0,
\end{aligned}
$$


or, with $g_{0}=3 f_{0}+f_{2}$ and $g_{2}=f_{0}-f_{2}$,

$$
g_{0}^{\prime \prime}=-3 \beta, \quad g_{2}^{\prime \prime}+8 g_{2}=-\beta .
$$

We remark that the same asymptotic system shows up in an asymptotic expansion of a solution of (1.24) when one attempts to find an approximate solution by an ansatz of the form (1.27) but with $u$ replaced by the first approximation of the asymptotic expansion for the solution of the linear homogeneous equation (1.3).

Recall that $\beta \in \mathcal{S}$, i.e. the space of rapidly decaying smooth functions, and that the Fourier transform maps $\mathcal{S}$ to itself. Taking the Fourier transform of this system we see that we obtain a system

$$
-\zeta^{2} \widehat{g}_{0}(\zeta)=-3 \widehat{\beta}(\zeta), \quad\left(-\zeta^{2}+8\right) \widehat{g}_{2}(\zeta)=-\widehat{\beta}(\zeta)
$$

This system has a solution in $\mathcal{S}$ if and only if $\widehat{\beta}(\zeta)$ has a double zero at $\zeta=0$ and simple zeros and $\zeta= \pm \sqrt{8}$, in which case the above method works.

Definition 1.3. In case $\widehat{\beta}(\zeta)$ has a double zero at $\zeta=0$ and simple zeros and $\zeta= \pm \sqrt{8}$, we define the functional $f_{i}[\beta]$ to be the fast decaying solution of the above system.

In what follows we may therefore assume that $\widehat{\beta}$ is supported in a small neighborhood of either 0 or $\sqrt{8}$ or $-\sqrt{8}$. If $\widehat{\beta}$ is supported near 0 , then the $f_{i}$ are actually growing, although the derivatives are bounded. In this case we modify the approach, taking into account that the solution decays for large frequencies. Let $f_{i}[\beta], i=0,2$, be the functionals defined by solving the system above depending on $\beta$; see Definition 1.3. Let us make the frequency decomposition

$$
\beta=\sum_{j=0}^{\infty} \beta_{j}, \quad \text { where } \quad \beta_{j}=P_{2^{-j}} \beta, \quad j \geq 0,
$$

the projection onto a dyadic region of frequencies $\sim 2^{-j}$. We now define

$$
w_{2, j}=\frac{1}{\rho} \sum_{i=0,2} f_{i}\left[\beta_{j}\right] F_{i}\left(v_{j}, \dot{v}_{j}\right), \quad \text { where } \quad v_{j}=P_{\leq \rho / 2^{j}} v, \quad \widehat{\beta}_{j}(\xi)=\chi_{1}\left(2^{j} \xi\right) \widehat{\beta}(\xi) ;
$$

here $\chi_{1}$ is supported in a neighborhood of 1 , which attempts to solve

$$
\left(\square_{\mathcal{H}}+1\right) w_{2, j} \sim \rho^{-1} \beta_{j}(\rho y) F_{0}\left(v_{j}\right)=\rho^{-1} P_{\sim \rho / 2^{j}} \beta(\rho y) F_{0}\left(P_{\leq \rho / 2^{j}} v\right) .
$$

We will sum only over values of $j$ for which $2^{j} \leq \rho^{1 / 2}$, and form

$$
w_{2}=\sum_{j=1}^{\infty} \chi_{0}\left(2^{j} / \rho^{1 / 2}\right) w_{2, j}
$$

where $\chi_{0} \in C_{0}^{\infty}$ is 1 in a neighborhood of the origin. The remainder

$$
\rho^{-1} P_{\leq \rho^{1 / 2}} \beta(\rho y) F_{0}(v)
$$

is easy to control since its easy to see that $P_{\leq \rho^{1 / 2}} \beta(\rho y)=\int e^{i y \rho \zeta} \chi\left(\zeta \rho^{1 / 2}\right) \hat{\beta}(\zeta) d \zeta$ satisfies

$$
\left|\partial_{y}^{k} P_{\leq \rho^{1 / 2}} \beta(\rho y)\right| \lesssim \rho^{(k+1) / 2-1}, \quad\left\|\partial_{y}^{k} P_{\leq \rho^{1 / 2}} \beta(\rho y)\right\|_{L_{y}^{2}} \lesssim \rho^{(k+1) / 2-3 / 2},
$$

which are bounded for $k \leq 1$.

What makes this argument work is that

$$
\left\|\beta_{j}(\rho y)\right\|_{L^{\infty}} \lesssim 1 / 2^{j}, \quad\left\|\beta_{j}(\rho y)\right\|_{\dot{H}^{n}} \lesssim\left(\rho / 2^{j}\right)^{n-1 / 2} / 2^{j}, \quad n=0,1 .
$$


Note that $\left\|v_{j}\right\|_{L^{\infty}} \lesssim\|v\|_{L^{\infty}}$, since the kernels of the projections are uniformly bounded in $L^{1}$. Since

$$
\left\|v-v_{j}\right\|_{L^{\infty}} \lesssim\left(\rho 2^{-j}\right)^{-1 / 2}\|v\|_{\dot{H}^{1}}
$$

it follows that

$$
\left\|\beta_{j}(\rho y) F_{0}\left(v_{j}\right)-\beta_{j}(\rho y) F_{0}(v)\right\|_{\dot{H}^{1}} \lesssim 2^{-j}\|v\|_{\dot{H}^{1}}\|v\|_{L^{\infty}}^{2},
$$

which can be summed over $j \geq 0$ to produce an error of the form (1.26).

For each fixed frequency the normal form is bounded and so is its error, so it remains to show that constants in the error bounds can be summed up for $2^{j} \leq \rho^{1 / 2}$ to produce an error bound of the same form. The details will be left to section 7 .

If $\widehat{\beta}$ is supported near $\pm \sqrt{8}$, then the best thing we can say is that $f_{0}(\rho y)$ and $f_{2}(\rho y)$ solving the above system are only bounded and more importantly their derivatives no longer decay. Therefore we no longer can assume that their derivatives with respect to $\rho$ are decaying when $|y|$ is bounded from below. In our situation, because $\beta$ is fast decaying, this may be overcome by multiplying by a cutoff $\chi\left(\rho^{a} y\right)$ for some $0<a<1$, where $\chi$ is 1 in a neighborhood of the origin. We will however instead take a different approach and obtain a new more general variable coefficient normal-form transformation that is a better approximation as long as $|y|$ is bounded from above. This is obtained by taking into account the $\rho$ derivatives of the system to obtain

$$
\begin{aligned}
\square_{\mathcal{H}} f_{0}-2 f_{0}-2 \partial_{\rho} f_{1}+2 f_{2} & =\beta(\rho y), \\
\square_{\mathcal{H}} f_{1}-6 f_{1}+6 \partial_{\rho} f_{0}-4 \partial_{\rho} f_{2}+6 f_{3} & =0, \\
\square_{\mathcal{H}} f_{2}-6 f_{2}+6 f_{0}+4 \partial_{\rho} f_{1}-6 \partial_{\rho} f_{3} & =0, \\
\square_{\mathcal{H}} f_{3}-2 f_{3}+2 f_{1}+2 \partial_{\rho} f_{2} & =0 .
\end{aligned}
$$

As before, starting with $g_{0}=3 f_{0}+f_{2}, g_{2}=f_{0}-f_{2}, g_{1}=f_{1}+3 f_{3}, g_{3}=f_{1}-f_{3}$, we get

$$
\begin{aligned}
\square_{\mathcal{H}} g_{0}-2 \partial_{\rho} g_{1} & =3 \beta, \\
\square_{\mathcal{H}} g_{1}+2 \partial_{\rho} g_{0} & =0, \\
\square_{\mathcal{H}} g_{2}-8 g_{2}-6 \partial_{\rho} g_{3} & =\beta, \\
\square_{\mathcal{H}} g_{3}-8 g_{3}+6 \partial_{\rho} g_{2} & =0 .
\end{aligned}
$$

Complexifying the above system we get that

$$
K_{1}=\left(g_{0}+i g_{1}\right) e^{i \rho} / 3, \quad K_{3}=\left(g_{2}+i g_{3}\right) e^{3 i \rho}
$$

give the system

$$
\begin{array}{lr}
\left(\square_{\mathcal{H}}+1\right) K_{1}=e^{i \rho} \beta(\rho y) & (0 \text { resonance equation }), \\
\left(\square_{\mathcal{H}}+1\right) K_{3}=e^{3 i \rho} \beta(\rho y) & ( \pm \sqrt{8} \text { resonance equation }) .
\end{array}
$$

We note that we only have to solve these equations asymptotically, which can be done with the stationary phase method. We hence want an asymptotic solution $K_{n}[\beta]$ that solves

$$
\left(\square_{\mathcal{H}}+1\right) K_{n}=e^{i n \rho} \beta(x)+\mathcal{E}_{K_{n}}
$$

where the error $\mathcal{E}_{n}[\beta]$ decays sufficiently fast. Here the functional $K_{n}[\beta]$ and error $\mathcal{E}_{n}[\beta]$ satisfy the same kinds of estimates as we had for the functionals $f_{i}[\beta]$ before; 
i.e. if $\beta$ is smooth and fast decaying we have

$$
\begin{aligned}
\left|\partial_{\rho}^{k} D_{y}^{l} \chi K_{i}\right| & \leqslant C_{k, l}, \quad\left|\partial_{\rho}^{k} D_{y}^{l} \chi \mathcal{E}_{K_{i}}\right| \leqslant \rho^{-1} C_{k, l}, \\
\|\left(\chi K_{i}, D_{y} \chi K_{i}, \partial_{\rho} \chi K_{i} \|_{B_{\rho}^{\infty}}\right. & \lesssim 1 .
\end{aligned}
$$

Here $\chi=\chi(y)$ is a smooth bump function in the $y$ variable.

\subsection{Proof of the main theorem in bootstrap form.}

Theorem 1.4 (Main energy bound in bootstrapping form). Suppose that the function $v$ solves the equation (1.6) with compactly supported initial data $\left(v_{0}, \dot{v}_{0}\right)$ at $\rho=1$. Then there exist universal constants $C_{0}, \epsilon_{0}>0,0<\delta<1 / 8$ such that if $\epsilon<\epsilon_{0}$ and

$$
\left\|\left(v, \dot{v}, \partial_{y} v\right)(1)\right\|_{H^{1}} \leq \epsilon,
$$

and one also assumes the time dependent bounds

$$
\left\|\left(v, \dot{v}, \rho^{-1} \partial_{y} v\right)(\rho)\right\|_{H^{1}} \leqslant 2 C_{0} \epsilon \rho^{\delta}, \quad \text { for } \quad 1 \leq \rho \leq T,
$$

then one also has the following improved time dependent bounds:

$$
\left\|\left(v, \dot{v}, \rho^{-1} \partial_{y} v\right)(\rho)\right\|_{H^{1}} \leqslant C_{0} \epsilon \rho^{\delta}, \quad \text { for } 1 \leq \rho \leq T .
$$

We now define a norm that is just slightly stronger than $L^{\infty}$ for high frequencies:

$$
\|v\|_{B_{\rho}^{\infty}}=\left\|P_{\leqslant \rho} v\right\|_{L^{\infty}}+\sum_{\lambda \geq \rho} \ln |\lambda / \rho|\left\|P_{\lambda} v\right\|_{L^{\infty}},
$$

where the sum is over dyadic $\lambda=2^{j}$. We prove in section 4 that the $L^{\infty}$ bound follows from the $H^{1}$ bound.

Proposition 1.5. Suppose that for some $0<\delta<1 / 8$,

$$
K=\sup _{1 \leq \rho \leq T} \rho^{-\delta}\left\|\left(v, \dot{v}, \rho^{-1} \partial_{y} v\right)(\rho)\right\|_{H^{1}}<\infty .
$$

Then

$$
\sup _{1 \leq \rho \leq T}\left\|\left(v, \dot{v}, \rho^{-1 / 2-\delta} \partial_{y} v\right)(\rho)\right\|_{B_{\rho}^{\infty}} \leq C K\left(1+K^{2}\right) .
$$

Theorem 1.1 follows from this.

It is clear that for times $\rho=1+O(1)$ one has the bound (1.65) from standard local existence theory. Furthermore, it is also clear that given the bound (1.65) up to time $T$, we may extend the solution to a later time $T+O(1)$ such that the weaker bound (1.64) holds. Therefore $T$ is not the maximal time for which (1.64) holds, and we can hence conclude that the estimate holds for $T=\infty$ so we have a global bound. Therefore, the remainder of the paper will be devoted to recovering the estimate (1.65) assuming that one has (1.63) as well as the estimates (1.64).

Because the $B_{\rho}^{\infty}$ follows from the $H^{1}$ it only remains to prove the $H^{1}$ estimate assuming the $B_{\rho}^{\infty}$. In proving the $H^{1}$ estimate for $v$ we will first subtract off a quadratic normal form $w_{1}$ and a cubic normal form $w_{2}$, depending on the nonlinearities, i.e. on the coefficients and on the solution $v$ and its $\rho$ derivative $\dot{v}$ :

$$
w_{1}=B((v, \dot{v}),(v, \dot{v})),
$$

where $B$ is a bilinear (nonlocal) operator given by (6.10) and

$$
w_{2}=T((w, \dot{w}),(w, \dot{w}),(w, \dot{w})), \quad \text { where } \quad w=v-w_{1}
$$


and $T$ is a trilinear (nonlocal) operator given in section 7. After subtracting these normal forms we are left with a remainder

$$
w_{0}=v-w_{1}-w_{2},
$$

satisfying

$$
\left(\square_{\mathcal{H}}+1\right) w_{0}=\mathcal{E}
$$

where the error $\mathcal{E}$ is controllable by the estimates in section 7

Proposition 1.6. For some $N$ we have

$$
\|\mathcal{E}\|_{H^{1}} \leq \frac{C}{\rho}\left(1+\|(v, \dot{v})\|_{B_{\rho}^{\infty}}\right)^{N}\|(v, \dot{v})\|_{B_{\rho}^{\infty}}^{2}\|(v, \dot{v})\|_{H^{1}},
$$

and with $D_{y}=-i \rho^{-1} \partial_{y}$,

$$
\sum_{i=1}^{2}\left\|\left(w_{i}, \dot{w}_{i}, D_{y} w_{i}\right)(\rho)\right\|_{H^{1}} \leq \frac{C}{\rho^{1 / 4}}\left(1+\|(v, \dot{v})\|_{B_{\rho}^{\infty}}\right)^{N}\|(v, \dot{v})\|_{B_{\rho}^{\infty}}\left\|\left(v, \dot{v}, D_{y} v\right)\right\|_{H^{1}} .
$$

The energy estimate (see section 2 ) for $w_{0}$ gives

(1.75) $\sup _{1 \leq \rho \leq T}\left\|\left(w_{0}, \dot{w}_{0}, D_{y} w_{0}\right)(\rho)\right\|_{H^{1}} \leq\left\|\left(w_{0}, \dot{w}_{0}, D_{y} w_{0}\right)(1)\right\|_{H^{1}}+\int_{1}^{T}\|\mathcal{E}\|_{H^{1}} d \rho$.

Now, assuming (1.64) it follows that

$$
\sup _{1 \leq \rho \leq T}\left\|\left(v, \dot{v}, D_{y} v\right)(\rho)\right\|_{B_{\rho}^{\infty}} \leq C_{1} \epsilon
$$

and

$$
\sum_{i=1}^{2}\left\|\left(w_{i}, \dot{w}_{i}, D_{y} w_{i}\right)(\rho)\right\|_{H^{1}} \leq \frac{C_{2} \epsilon}{\rho^{1 / 4}}\left\|\left(v, \dot{v}, D_{y} v\right)\right\|_{H^{1}},
$$

and therefore since $w_{0}=v-w_{1}-w_{2}$,

$$
\|\mathcal{E}\|_{H^{1}} \leq \frac{C_{3} \epsilon^{2}}{\rho}\|(v, \dot{v})\|_{H^{1}} \leq \frac{C_{4} \epsilon^{2}}{\rho}\left\|\left(w_{0}, \dot{w}_{0}\right)\right\|_{H^{1}} .
$$

Hence

$$
\begin{aligned}
\sup _{1 \leq \rho \leq T}\left\|\left(w_{0}, \dot{w}_{0}, D_{y} w_{0}\right)(\rho)\right\|_{H^{1}} \leq\left\|\left(w_{0}, \dot{w}_{0}, D_{y} w_{0}\right)(1)\right\|_{H^{1}} \\
+\int_{1}^{T} \frac{C_{4} \epsilon^{2}}{\rho}\left\|\left(w_{0}, \dot{w}_{0}, D_{y} w_{0}\right)\right\|_{H^{1}} d \rho .
\end{aligned}
$$

It finally follows from this that

$$
\sup _{1 \leq \rho \leq T}\left\|\left(w_{0}, \dot{w}_{0}, \rho^{-1} \partial_{y} w_{0}\right)(\rho)\right\|_{H^{1}} \leq \rho^{C_{4} \epsilon^{2}}\left\|\left(w_{0}, \dot{w}_{0}, \partial_{y} w_{0}\right)(1)\right\|_{H^{1}} .
$$

Since also

$$
\left\|\left(w_{0}, \dot{w}_{0}, \partial_{y} w_{0}\right)(1)\right\|_{H^{1}} \leq(1+C \epsilon)\left\|\left(v, \dot{v}, \partial_{y} v\right)(1)\right\|_{H^{1}} \leq \frac{5}{4} \epsilon,
$$

if $\epsilon$ is sufficiently small by (1.63), it follows that

$$
\left\|\left(v, \dot{v}, D_{y} v\right)(1)\right\|_{H^{1}} \leq \frac{5}{4}\left\|\left(w_{0}, \dot{w}_{0}, D_{y} w_{0}\right)(1)\right\|_{H^{1}} \leq\left(\frac{5}{4}\right)^{2} \epsilon \rho^{C_{4} \epsilon^{2}} .
$$


If $\epsilon$ is so small that $C_{4} \epsilon^{2} \leq \delta$ this proves (1.65) and concludes the proof of the theorem.

\section{Hyperbolic COORDinAtes AND EnERgy Estimates}

In this section, we set up a convenient set of coordinates for proving decay estimates of the form (1.2). Because we are assuming that the initial data (1.1b)(1.1c) for our problem is compactly supported, by time translating the Cauchy problem forward by an $O(1)$ amount and using finite speed of propagation, it suffices to do all of our analysis in the interior of the forward cone $|x| \leqslant t$. That is, without loss of generality we may assume that the initial data $(1.1 \mathrm{~b}-1.1 \mathrm{c})$ is defined at $t=1$ and its support is contained in the set $|x| \leqslant \frac{1}{2}$.

2.1. Hyperbolic coordinates. As is well known, this forward region is foliated by hyperboloids const $=\rho=\sqrt{t^{2}-|x|^{2}}$, and one may introduce the following set of hyperbolic coordinates:

$$
x=\rho \sinh (y), \quad t=\rho \cosh (y) .
$$

By the time shifting setup of the last paragraph and finite speed of propagation, we have that the support of our solution $u(t, x)$ is contained in the region $1 \leqslant \rho<$ $\infty$. Our first goal is to transfer the Cauchy problem (1.1) in this new system of coordinates.

The formulas for the coordinate derivatives of (1.5) are as follows:

$$
\partial_{y}=t \partial_{x}+x \partial_{t}, \quad \partial_{\rho}=\frac{1}{\sqrt{t^{2}-|x|^{2}}}\left(t \partial_{t}+x \partial_{x}\right) .
$$

In particular, notice that the derivative $\partial_{y}$ is nothing other than the $1 \mathrm{D}$ Lorentz boost, which commutes with the linear part of equation (1.1a). It will be estimates involving this weighted vector-field that are ultimately responsible for the bulk of our proof of the decay estimate (1.2).

In the coordinates (1.5) the Minkowski metric takes the following simple form

$$
-d t^{2}+d x^{2}=-d \rho^{2}+\rho^{2} d y^{2} .
$$

Therefore, the linear Klein-Gordon operator takes the form

$$
\partial_{t}^{2}-\partial_{x}^{2}+1=\partial_{\rho}^{2}+\frac{1}{\rho} \partial_{\rho}-\frac{1}{\rho^{2}} \partial_{y}^{2}+1 .
$$

The RHS of this last formula is further simplified via conjugation by the weight $\rho^{\frac{1}{2}}$. That is, one has the identity

$$
\rho^{\frac{1}{2}}\left[\partial_{\rho}^{2}+\frac{1}{\rho} \partial_{\rho}-\frac{1}{\rho^{2}} \partial_{y}^{2}+1\right](u)=\left[\partial_{\rho}^{2}-\frac{1}{\rho^{2}} \partial_{y}^{2}+\frac{1 / 4}{\rho^{2}}+1\right]\left(\rho^{\frac{1}{2}} u\right) .
$$

Therefore, introducing the new quantity $v=\rho^{\frac{1}{2}} u$ the equation (1.1a) becomes

$$
\left(\partial_{\rho}^{2}-\frac{1}{\rho^{2}} \partial_{y}^{2}+1+\frac{1 / 4}{\rho^{2}}\right) v=\frac{\alpha_{0}}{\rho^{\frac{1}{2}}} v^{2}+\frac{\beta(\rho \sinh (y))}{\rho} v^{3}+\frac{\beta_{0}}{\rho} v^{3} .
$$

It will be convenient for us to further simplify the expression for the nonlinear potential $\beta$. Using the fact that this is a smooth function, we may write

$$
\beta(\rho \sinh (y))-\beta(\rho y)=\mathcal{R}_{\beta}(\rho, y) .
$$


Therefore, we may write the equation for $v$ in the form

$$
\left(\partial_{\rho}^{2}-\frac{1}{\rho^{2}} \partial_{y}^{2}+\left(1+\frac{1}{4 \rho^{2}}\right)\right) v=\frac{\alpha_{0}}{\rho^{\frac{1}{2}}} v^{2}+\frac{\beta(\rho y)}{\rho} v^{3}+\frac{\beta_{0}}{\rho} v^{3}+\frac{1}{\rho} \mathcal{R}_{\beta}(\rho, y) v^{3} .
$$

Remark 2.1. In the sequel, the reader is encouraged to envision the original equation (1.1a) as transforming under the change of coordinates (1.5) into equation (2.5) without the remainder term $\rho^{-1} \mathcal{R}_{\beta}(\rho, y) v^{3}$. Estimates involving this remainder will always have a lot of room in the norms we are using. In fact, by the lemma below it decays two powers $\rho^{-2}$ faster than $\rho^{-1} \beta(\rho y) v^{3}$. Similarly the term $v /\left(4 \rho^{2}\right)$ will decay two powers $\rho^{-2}$ faster than the term $v$. The reader should therefore keep in mind the simplified equation

$$
\left(\partial_{\rho}^{2}-\frac{1}{\rho^{2}} \partial_{y}^{2}+1\right) v=\frac{\alpha_{0}}{\rho^{\frac{1}{2}}} v^{2}+\frac{\beta(\rho y)}{\rho} v^{3}+\frac{\beta_{0}}{\rho} v^{3},
$$

and at some places we will just prove the estimates for this simplified equation since it is easy to estimate the additional terms.

Lemma 2.2. For $0 \leq m \leq 1$,

$$
\left|\partial_{y}^{k} \partial_{\rho}^{m}(\beta(\rho \sinh (y))-\beta(\rho y))\right| \leq \frac{C_{k} \rho^{k}}{\rho^{2+m}} \sum_{\ell \leq k+1+m} \sup _{|z| \geq \rho|y|}\left|\beta^{(\ell)}(z)\right|(1+|z|)^{3+m}
$$

Proof. First note that the estimates for $m=1$ follow from the estimate for $m=0$ applied to $z \beta^{\prime}(z)$ so we may assume that $m=0$. If $|y| \geq 1$, then each of the terms separately is bounded by the right hand side (in fact with 2 replaced by any integer). For the second term this just follows from that $\rho|y| \geq \rho$ then. For the first term one may also have to use that when $|y| \geq 1, e^{|y|} / 4 \leq|\sinh (y)| \leq|\cosh (y)| \leq e^{|y|}$.

For $0 \leq y \leq 1$ we write $\sinh (y)=y+y^{3} b(y)$ for some smooth function $b(y)$. With $x=\rho y$ and $z=x+\rho^{-2} x^{3} b(x / \rho) t$ we have

$\beta(\rho \sinh (y))-\beta(\rho y)=\int_{\rho y}^{\rho \sinh (y)} \beta^{\prime}(z) d z=\rho^{-2} x^{3} b(x / \rho) \int_{0}^{1} \beta^{\prime}\left(x+\rho^{-2} x^{3} b(x / \rho) t\right) d t$,

which is bounded by $C \rho^{-2} x^{3} \sup _{z>x}\left|\beta^{\prime}(z)\right| \leq C \rho^{-2} \sup _{z>x}\left|\beta^{\prime}(z)\right| z^{3}$. This proves the lemma for $k=0$. The lemma for $k \geq 1$ follows since

$$
\left|\partial_{x}^{k}\left(\rho^{-2} x^{3} b(x / \rho)\right)\right| \leq C_{k}, \quad k \geq 1, \quad|x| \leq \rho .
$$

2.2. Energy estimates. Our next order of business is to record how bounds in terms of the energy norm may be recovered through Duhamel's principle for the linear operator on the LHS of equation (2.5).

Lemma 2.3 (Duhamel estimate). Suppose that $w$ solves the equation inside the time slab $[1, T] \times \mathbb{R}$ :

$$
\left(\partial_{\rho}^{2}-\frac{1}{\rho^{2}} \partial_{y}^{2}+\left(1+\frac{1}{4 \rho^{2}}\right)\right) w=F
$$


Then for $m+n \leq 1$ and $k \geq 0$ one has the bounds

$$
\begin{aligned}
\sup _{1 \leqslant \rho \leqslant T} & \left\|\left(\rho^{-1} \partial_{y}\right)^{m} \partial_{\rho}^{n}\left(w, \dot{w}, \rho^{-1} \partial_{\rho} w\right)(\rho)\right\|_{H^{k}} \\
& \lesssim\left\|\left(\rho^{-1} \partial_{y}\right)^{m} \partial_{\rho}^{n}\left(w, \dot{w}, \rho^{-1} \partial_{y} w\right)(1)\right\|_{H^{k}}+\int_{1}^{T}\left\|\left(\rho^{-1} \partial_{y}\right)^{m} \partial_{\rho}^{n} F(\rho)\right\|_{H^{k}} d \rho .
\end{aligned}
$$

Proof. The energy estimate (2.11) is essentially standard. Multiplying the equation (2.10) by $\partial_{\rho} w$ and integrating by parts on all possible time slabs inside $\mathbb{R} \times[1, S]$ and using Young's inequality on the product $\|F(\rho)\|_{L^{2}} \cdot\left\|\partial_{\rho} w\right\|_{L^{2}}$ to absorb the second factor to the LHS we have

$$
\begin{aligned}
\sup _{1 \leqslant \rho \leqslant S}\left\|\left(w, \dot{w}, \rho^{-1} \partial_{y} w\right)(\rho)\right\|_{L^{2}}^{2}+\int_{1}^{S}\left\|\rho^{-1} \partial_{y} w(\rho)\right\|_{L^{2}}^{2} \frac{d \rho}{\rho} & \\
& \lesssim\left\|\left(w, \dot{w}, \partial_{y} w\right)(1)\right\|_{L^{2}}^{2}+\left(\int_{1}^{S}\|F(\rho)\|_{L^{2}} d \rho\right)^{2} .
\end{aligned}
$$

We will not use space-time integrals like the one on the LHS in this work, so we discard it in estimate (2.11). The $\partial_{y} v$ differentiated terms in the estimate (2.11) are likewise bounded by integrating the $\partial_{y}$ derivative of equation (2.10) multiplied by the quantity $\partial_{\rho} \partial_{y} w$. To get an estimate for additional derivatives we first apply $\rho^{-1} \partial_{y}$ to the equation and then multiply with $\partial_{\rho}\left(\rho^{-1} \partial_{y} w\right)$ to get an additional commutator term in the left hand side:

$$
\rho^{-2} \partial_{\rho} \partial_{y} w \partial_{\rho}\left(\rho^{-1} \partial_{y} w\right)=\rho^{-1}\left(\partial_{\rho}\left(\rho^{-1} \partial_{y} w\right)\right)^{2}-\rho^{-5 / 2} \partial_{y} w \rho^{-1 / 2} \partial_{\rho}\left(\rho^{-1} \partial_{y} w\right),
$$

where the first term gives a positive spacetime contribution to the energy and the second can be controlled by the first and the space time part and the lower parts of the energy using Cauchy-Schwarz. Similarly applying $\partial_{\rho}$ to the equation and multiplying with $\partial_{\rho}^{2} w$ give an additional commutator term in the left hand side:

$$
2 \rho^{-3} \partial_{y}^{2} w \partial_{\rho}^{2} w=2 \rho^{-5} \partial_{y}^{2} w \partial_{y}^{2} w-2 \rho^{-3} \partial_{y}^{2} w w,
$$

where as before the first term gives a positive space time contribution to the energy and the last term after integrating parts gives a positive contribution as well.

Finally, we wish to formalize the relationship between the Duhamel estimate (2.11) and the energy norm we are working with. In our work, we will need to let this norm grow at a slow rate due to long range corrections. The space which keeps track of this is the following.

Definition 2.4. We define the source term space $\mathcal{S}_{\delta}[1, T]$ of index $\delta$ up to time $T$ to be completion of test functions under the norm:

$$
\begin{gathered}
\|F\|_{\mathcal{S}_{\delta}[1, T]}=\sup _{1 \leqslant \rho \leqslant T} \rho^{1-\delta}\|F(\rho)\|_{H^{1}}, \\
\left\|\rho^{\delta} v\right\|_{L^{\infty}\left([1, T] ; H^{1}\right)}=\sup _{1 \leqslant \rho \leqslant T} \rho^{\delta}\|v(\rho)\|_{H^{1}} .
\end{gathered}
$$


Based on this definition and the estimate (2.11), the following is immediate:

Lemma 2.5 (Duhamel estimates from source term spaces). Let $w$ solve the equation (2.10). Then one has the following uniform bounds for any time slab $[1, T]$ :

$$
\sup _{1 \leqslant \rho \leqslant T} \rho^{-\delta}\left\|\left(w, \dot{w}, \rho^{-1} \partial_{y} w\right)(\rho)\right\|_{H^{1}} \lesssim \delta^{-1}\|F\|_{\mathcal{S}_{\delta}[1, T]} .
$$

We remark that there is a lowest order energy estimate also for the nonlinear equation which shows that the $L^{2}$ norm is bounded. However, this is no longer the case for the differentiated equation, and higher energies will grow. The nonlinear terms will be too large to treat as a right hand side in the energy estimate, but we will first remove them by normal-form transformations and after that apply the energy estimate to the resulting equation.

We also have some nonlinear energy estimates:

Lemma 2.6 (Nonlinear energy estimates). Suppose that $v$ solves the equation inside the time slab $[1, T] \times \mathbb{R}$ :

$$
\left(\partial_{\rho}^{2}-\frac{1}{\rho^{2}} \partial_{y}^{2}+\left(1+\frac{1}{4 \rho^{2}}\right)\right) v+\frac{\alpha_{0}}{\rho^{1 / 2}} v^{2}+\frac{\beta(\rho y)}{\rho} v^{3}=0
$$

Suppose also that for $1 \leq \rho \leq T$,

$$
\|(v, \dot{v})(\rho, \cdot)) \|_{L^{\infty}} \leq K \leq\left(1+16\left(\left|\alpha_{0}\right|+\sup _{z}\left[|\beta(z)|+|z|\left|\beta^{\prime}(z)\right|\right]^{1 / 2}\right)\right)^{-1} .
$$

Then for $1 \leq \rho \leq T$ we have

$$
\sup _{1 \leqslant \rho \leqslant T}\left\|\left(v, \dot{v}, \rho^{-1} \partial_{\rho} v\right)(\rho)\right\|_{L^{2}} \lesssim\left\|\left(v, \dot{v}, \rho^{-1} \partial_{y} v\right)(1)\right\|_{L^{2}} .
$$

Proof. Let

$$
E_{0}(\rho)=\int \frac{1}{2} v_{\rho}^{2}+\frac{1}{2 \rho^{2}} v_{y}^{2}+\frac{1}{2}\left(1+\frac{1}{4 \rho^{2}}\right) v^{2}+\frac{\alpha_{0}}{3 \rho^{1 / 2}} v^{3}+\frac{\beta}{4 \rho} v^{4} d y
$$

We note that by assumption the last two terms are bounded by the third so $E_{0}$ is equivalent to the norm $\left\|\left(v, \dot{v}, \rho^{-1} \partial_{\rho} v\right)(\rho)\right\|_{L^{2}}$, and in particular positive. After integrating one term by parts in $y$ and using the equation we get

$$
E_{0}^{\prime}(\rho)=\int-\frac{1}{\rho^{3}}\left(v_{y}^{2}+v^{2} / 4\right)-\frac{\alpha_{0}}{6 \rho^{3 / 2}} v^{3}-\frac{\beta_{1}}{4 \rho^{2}} v^{4} d y,
$$

where

$$
\beta_{1}(z)=\beta(z)-z \beta^{\prime}(z)
$$

Hence

$$
E_{0}^{\prime}(\rho) \leq\left(\frac{K}{6 \rho^{3 / 2}}+\frac{K^{2}}{\rho^{2}}\right) E_{0}(\rho)
$$

from which the bound $E_{0}(\rho) \lesssim E_{0}(1)$ follows. 


\section{LitTlewood-PAley theory, FunCtion spaces}

We begin this section with some standard notation.

3.1. Littlewood-Paley setup. Our next task is to set up the standard LittlewoodPaley theory in the $y$ coordinate. First, we introduce the spatial Fourier transform:

$$
\widehat{v}(\xi)=\int_{\mathbb{R}} e^{-i \xi y} v(y) d y
$$

In this normalization, the Plancherel theorem reads $\|v\|_{L^{2}(d y)}=(2 \pi)^{\frac{1}{2}}\|\widehat{v}\|_{L^{2}(d \xi)}$. We let $p_{\lambda}$ be a dyadically indexed partition of unity in the frequency variable such that $p_{1} \equiv 1$ in a neighborhood of $\xi=0$, and such that for $\lambda \geq 1$,

$$
p_{\lambda}(\xi)=p_{0}\left(\lambda^{-1} \xi\right),
$$

for some basic annular cutoff $p_{0}$. We will not fix once and for all this collection of cutoffs $p_{\lambda}$, but rather we shall let them vary from line to line in the sequel. This will relieve us from having to consecutively label sequence cutoffs whose supports may increase in the various proofs which follow. As usual, we denote by $P_{\lambda}$ the corresponding convolution operator in the physical variable $y$.

It will often be convenient for us to combine these dyadic cutoffs in various ways. We use a standard notation for this:

$$
P_{\lesssim \lambda}=\sum_{\sigma: \sigma \lesssim \lambda} P_{\sigma}, \quad P_{\gtrsim \lambda}=\sum_{\sigma: \lambda \lesssim \sigma} P_{\sigma},
$$

and so on depending on the context. In such expressions as the above, it is always assumed that we are summing over consecutive dyadic indices. We always assume that we are summing over dyadic values larger than one, unless otherwise specified. The reader should keep in mind that the problem at hand is not scale invariant.

Notice that all of the operators, $P_{\lambda}, P_{\lesssim \lambda}$, and $P_{\gtrsim \lambda}$ are given by $L^{1}$ convolution kernels with uniform bounds. Therefore their action is uniformly bounded on all $L^{p}$ spaces including $L^{1}$ and $L^{\infty}$.

Associated with cutoffs of the form $P_{\lambda}$, one has Bernstein's inequality:

$$
\left\|P_{\lambda} v\right\|_{L^{\infty}} \lesssim \lambda^{\frac{1}{2}}\left\|P_{\lambda} v\right\|_{L^{2}}
$$

This may be rewritten as the local Sobolev type bound:

$$
\left\|P_{\lambda} v\right\|_{L^{\infty}} \lesssim \lambda^{-\frac{1}{2}}\left\|P_{\lambda} \partial_{y} v\right\|_{L^{2}}
$$

These two estimates will be used many times in the sequel.

3.2. Function spaces. In this paper, most of our work will be to bound a weighted energy norm of the solution $v$. One may introduce the usual $H^{1}$ inhomogeneous Sobolev space of functions in the $y$ variable:

$$
\|v\|_{H^{1}}=\|v\|_{L^{2}}+\left\|\partial_{y} v\right\|_{L^{2}} \approx\left(\sum_{\lambda} \lambda^{2}\left\|P_{\lambda} v\right\|_{L^{2}}^{2}\right)^{\frac{1}{2}} \approx\left(\sum_{\lambda}\left\|P_{\lambda} v\right\|_{H^{1}}^{2}\right)^{\frac{1}{2}}
$$


It will also be necessary for us to have a version of $L^{\infty}$ that is strong enough to obey estimates for a certain brand of singular integral operators that are common in our work. This norm must also be weak enough that it will allow us to recapture it on certain frequency ranges using nondyadic decompositions. Therefore, we construct the following time dependent hybrid $L^{\infty}$ space:

$$
\|v\|_{B_{\rho}^{\infty}}=\left\|P_{\leqslant \rho} v\right\|_{L^{\infty}}+\sum_{\lambda \geq \rho} \ln |\lambda / \rho|\left\|P_{\lambda} v\right\|_{L^{\infty}} .
$$

Note that by Bernstein's inequality and Cauchy-Schwarz, $\|v\|_{B_{\infty}^{\infty}} \lesssim\|v\|_{H^{1}}$.

In the sequel, we shall also use the notation $\left\|\left(v, \dot{v}, \rho^{-1} \partial_{y} v\right)\right\|_{H^{1}}$, etc., to denote that norm applied to the triple $\left(v, \partial_{\rho} v, \rho^{-1} \partial_{y} v\right)$ as a direct sum.

We now record a few simple estimates involving the relationship between these $L^{\infty}$ type spaces and the energy type norms defined above, as well as an algebra estimate:

Lemma 3.1 (Time dependent $L^{\infty}$ type bounds). For test functions $v$ one has the following uniform bounds (e.g. uniform in $0 \leqslant \sigma$ ):

$$
\begin{aligned}
\|v\|_{L^{\infty}} & \lesssim\|v\|_{H^{1}}^{1 / 2}\|v\|_{L^{2}}^{1 / 2} \\
\left\|P_{\geq \rho^{\sigma}} v\right\|_{L^{\infty}} & \lesssim \rho^{-\sigma / 2}\|v\|_{H^{1}}, \\
\left\|P_{\supset^{\sigma}} \partial_{y}^{k} v\right\|_{L^{\infty}} & \lesssim \rho^{(k-1 / 2) \sigma}\|v\|_{H^{1}}, \quad k \geq 1 \\
\left\|P_{\geq \rho^{\sigma}} v\right\|_{B_{\rho}^{\infty}} & \lesssim \rho^{-\sigma / 2}\|v\|_{H^{1}} \\
\left\|\rho^{-1} \partial_{y} P_{\leqslant \lambda} v\right\|_{L^{\infty}} & \lesssim \frac{\lambda^{\frac{1}{2}}}{\rho}\|v\|_{H^{1}} .
\end{aligned}
$$

Moreover,

$$
\begin{aligned}
\left\|P_{\sim \lambda} u\right\|_{L^{\infty}} & \lesssim\|u\|_{L^{\infty}}, \\
\left\|P_{\leq \lambda} u\right\|_{L^{\infty}} & \lesssim\|u\|_{L^{\infty}}, \\
\left\|\partial_{y} P_{\leq \lambda} u\right\|_{L^{\infty}} & \lesssim \lambda\|u\|_{L^{\infty}}, \\
\|u \cdot v\|_{B_{\rho}^{\infty}} & \lesssim\|u\|_{B_{\rho}^{\infty}} \cdot\|v\|_{B_{\rho}^{\infty}}, \\
\left\|\left[\partial_{\rho}, P_{\leq c \rho}\right] u\right\|_{L^{\infty}} & \lesssim \frac{1}{c \rho}\left\|P_{\sim_{c \rho}} u\right\|_{L^{\infty}}, \\
\left\|\left[\partial_{\rho}, P_{\leq c \rho}\right] u\right\|_{H^{1}} & \lesssim \frac{1}{c \rho}\|u\|_{H^{1}} .
\end{aligned}
$$

Remark 3.2. The first bound above shows that for the most part the norm $H^{1}$ with slow growth will give $L^{\infty}$ type control of the solution $v$. However, for very low frequencies in the range $|\xi| \lesssim \rho^{2 \delta}$, we will need to use an additional argument to gain $L^{\infty}$ bounds. This will not be based on energy type spaces (see section 4 below), but will follow from a direct manipulation of the equation (2.5) restricted to low frequencies. 
Proof of (3.9)-(3.14). The proof of estimates of this type is standard and essentially boils down to quoting the estimates (3.2)-(3.3). We begin with (3.9). Summing over (3.3) we have

$$
\begin{aligned}
& \left\|P_{\geq \rho^{\sigma}} v\right\|_{B_{\rho}^{\infty}} \lesssim \sum_{\rho^{\sigma} \lesssim \lambda \lesssim \rho}\left\|P_{\lambda} v\right\|_{L^{\infty}}+\sum_{\rho \lesssim \lambda} \ln |\lambda / \rho|\left\|P_{\lambda} v\right\|_{L^{\infty}} \\
& \lesssim \sum_{\rho^{\sigma} \lesssim \lambda \lesssim \rho} \lambda^{-\frac{1}{2}} \lambda\left\|\widetilde{P}_{\lambda} v\right\|_{L^{2}}+\sum_{\rho \lesssim \lambda} \ln |\lambda / \rho| \lambda^{-\frac{1}{2}} \lambda\left\|\widetilde{P}_{\lambda} v\right\|_{L^{2}} \\
& \lesssim\left[\left(\sum_{\rho^{\sigma} \lesssim \lambda \lesssim \rho} \lambda^{-1}\right)^{\frac{1}{2}}+\left(\sum_{\rho \lesssim \lambda}(\ln |\lambda / \rho|)^{2} \lambda^{-1}\right)^{\frac{1}{2}}\right] \cdot\left(\sum_{\rho^{\sigma} \lesssim \lambda \lesssim \rho} \lambda^{2}\left\|\widetilde{P}_{\lambda} v\right\|_{L^{2}}^{2}\right)^{\frac{1}{2}} \\
& \lesssim\left(\rho^{-\sigma / 2}+\rho^{-1 / 2}\right)\|v\|_{H^{1}} .
\end{aligned}
$$

The proof of (3.10) follows from similar dyadic summing arguments. We leave the details to the reader.

The proof of (3.11) and (3.12) follows from the fact that the convolution kernels of $P_{\sim \rho}$ and $P_{\leqslant \rho}$ are uniformly $L^{1}$, and (3.15) follows from the same argument. Finally, let us prove the algebra estimate (3.14). This follows from a standard "trichotomy". The first step is to decompose the frequencies of the product dyadically. This only needs to be done for frequency blocks bigger than size $\rho$. That is, we first make the rough decomposition:

$$
u \cdot v=P_{\leqslant \rho}(u \cdot v)+P_{\geqslant \rho}(u \cdot v) .
$$

For the first term in this last expression, we have

$$
\left\|P_{\leqslant \rho}(u \cdot v)\right\|_{L^{\infty}} \lesssim\|u\|_{L^{\infty}} \cdot\|v\|_{L^{\infty}} .
$$

For the RHS of this last line, we easily have a bound in terms of $\|u\|_{B_{\rho}^{\infty}} \cdot\|v\|_{B_{\rho}^{\infty}}$ by expanding into frequencies and using the triangle inequality.

It remains to show (3.14) for the second term on the RHS of (3.18). This will be done by further decomposing this product into all frequencies $\rho \leqslant \lambda$ :

$$
\begin{gathered}
P_{\geqslant \rho}(u \cdot v)=\sum_{\rho \leqslant \lambda} P_{\lambda}\left[P_{\lambda} u \cdot P_{\ll \lambda} v\right]+\sum_{\rho \leqslant \lambda} P_{\lambda}\left[P_{\ll \lambda} u \cdot P_{\lambda} v\right] \\
\quad+\sum_{\rho \leqslant \lambda} P_{\lambda} \sum_{\mu_{1} \sim \mu_{2} \gtrsim \lambda} P_{\mu_{1}} u \cdot P_{\mu_{2}} v \\
=B_{1}+B_{2}+B_{3} .
\end{gathered}
$$

The proof of (3.14) for the terms $B_{1}$ and $B_{2}$ is essentially symmetric (one only needs to keep the weight function with the first factor). We focus on bounding $B_{1}$, and leave the details of the other to the reader. The multipliers $P_{\lambda}$ and $P_{\ll \lambda}$ are bounded on $L^{\infty}$. Therefore we have

$$
\begin{aligned}
\left\|B_{1}\right\|_{B_{\rho}^{\infty}} & \lesssim \sum_{\rho \leqslant \lambda} \ln \left(\frac{\lambda}{\rho}\right)\left\|P_{\lambda} u \cdot P_{\ll \lambda} v\right\|_{L^{\infty}} \\
& \leqslant \sup _{\lambda}\left\|P_{\ll \lambda} v\right\|_{L^{\infty}} \cdot \sum_{\rho \leqslant \lambda} \ln \left(\frac{\lambda}{\rho}\right)\left\|P_{\lambda} u\right\|_{L^{\infty}} \\
& \lesssim\|u\|_{B_{\rho}^{\infty}} \cdot\|v\|_{B_{\rho}^{\infty}} .
\end{aligned}
$$


Finally, we need to prove the bound (3.14) for the term $B_{3}$. By again using the boundedness of various Fourier cutoffs on $L^{\infty}$ we have

$$
\begin{aligned}
\left\|B_{3}\right\|_{B_{\rho}^{\infty}} & \lesssim \sum_{\lambda \geqslant \rho} \ln \left(\frac{\lambda}{\rho}\right) \sum_{\mu_{1} \sim \mu_{2} \gtrsim \lambda}\left\|P_{\mu_{1}} u \cdot P_{\mu_{2}} v\right\|_{L^{\infty}} \\
& \lesssim \sum_{\mu_{1} \sim \mu_{2} \gtrsim \rho} \sum_{\rho \leqslant \lambda \leqslant \mu_{1}} \ln \left(\frac{\lambda}{\rho}\right)\left\|P_{\mu_{1}} u \cdot P_{\mu_{2}} v\right\|_{L^{\infty}} \\
& \lesssim \sum_{\mu_{1} \sim \mu_{2} \gtrsim \rho} \ln \left(\frac{\mu_{1}}{\rho}\right) \ln \left(\frac{\mu_{2}}{\rho}\right)\left\|P_{\mu_{1}} u \cdot P_{\mu_{2}} v\right\|_{L^{\infty}} \\
& \lesssim \sup _{\mu_{1}}\left\|P_{\mu_{1}} v\right\|_{L^{\infty}} \ln \left(\frac{\mu_{1}}{\rho}\right) \cdot \sum_{\mu_{2} \gtrsim \rho} \ln \left(\frac{\mu_{2}}{\rho}\right)\left\|P_{\mu_{2}} u\right\|_{L^{\infty}} \\
& \lesssim\|u\|_{B_{\rho}^{\infty}} \cdot\|v\|_{B_{\rho}^{\infty}} .
\end{aligned}
$$

This completes the proof of (3.14).

\section{Decay estimates}

4.1. Decay estimates. In addition to energy estimate, as usual we also need decay estimates. The decay estimates for high frequencies can be obtained from the energy bounds of higher derivatives, essentially using (3.7). The decay estimates for low frequencies can be obtained directly from integrating the equation, provided the decay of the larger frequencies are established. In this section we will show that

Theorem 4.1. Suppose that for some $0<\delta<1 / 8$,

$$
K=\sup _{1 \leq \rho \leq T} \rho^{-\delta}\left\|\left(v, \dot{v}, \rho^{-1} \partial_{y} v\right)(\rho)\right\|_{H^{1}}<\infty .
$$

Then

$$
\sup _{1 \leq \rho \leq T}\left\|\left(v, \dot{v}, \rho^{-1 / 2-\delta} \partial_{y} v\right)(\rho)\right\|_{B_{\rho}^{\infty}} \lesssim K\left(1+K^{2}\right) .
$$

Proof. The estimate for $(v, \dot{v})$ follows from Lemma 4.3 below. The estimate for $\rho^{-1} \partial_{y} v$ follows from that of $\left\|\partial_{y} v\right\|_{L^{\infty}} \lesssim\left\|\partial_{y} v\right\|_{L^{2}}^{1 / 2}\left\|\partial_{y} v\right\|_{H^{1}}^{1 / 2} \lesssim K \rho^{\delta+1 / 2}$.

Lemma 4.2 (Low frequency decay estimate). Suppose that $w$ solves the equation inside the time slab $[1, T] \times \mathbb{R}$ :

$$
\partial_{\rho}^{2} w-\frac{1}{\rho^{2}} \partial_{y}^{2} w+\left(1+\frac{1}{4 \rho^{2}}\right) w+\frac{\alpha_{0}}{\rho^{1 / 2}} w^{2}-\frac{\beta_{0}}{\rho} w^{3}+\frac{\beta_{1}}{\rho} w \dot{w}^{2}=F .
$$

Suppose also that $P_{\lambda} w=0$, for $\lambda \geq \rho^{\sigma}$ and with $3 \sigma / 2+\delta<1,1 / 2>\delta>0, \sigma>0$,

$$
K=\sup _{1 \leq \rho \leq T} \rho^{-\delta}\|(w, \dot{w})(\rho)\|_{H^{1}}<\infty
$$

Then

$$
\sup _{1 \leqslant \rho \leqslant T}\|(w, \dot{w})(\rho)\|_{L^{\infty}} \lesssim K\left(1+K^{2 \delta /(1-2 \delta)}\right)+\int_{1}^{T}\|F(\rho)\|_{L^{\infty}} d \rho
$$


Proof. To begin with we note that for $1 \leq \rho \leq T$,

$$
\|(w, \dot{w})(\rho)\|_{L^{\infty}} \lesssim K \rho^{\delta}, \quad\left\|\partial_{y}^{2} w(\rho)\right\|_{L^{\infty}} \lesssim K \rho^{\delta+3 \sigma / 2} .
$$

The first inequality follows from (4.4) and the Sobolev estimate; the second also uses the assumed high frequency cutoff on $w$ :

$$
\left\|\partial_{y}^{2} w\right\|_{L^{\infty}} \lesssim\left\|\partial_{y}^{2} w\right\|_{L^{2}}^{1 / 2}\left\|\partial_{y}^{2} w\right\|_{H^{1}}^{1 / 2} \lesssim \rho^{3 \sigma / 2}\|w\|_{H^{1}}^{1 / 2}
$$

We have

$$
\partial_{\rho}\left(G(w, \rho) \frac{\dot{w}^{2}}{2}+H(w, \rho)\right)=G \dot{w}\left(\ddot{w}+\frac{G_{w}}{2 G} \dot{w}^{2}+\frac{H_{w}}{G}\right)+G_{\rho} \frac{\dot{w}^{2}}{2}+H_{\rho},
$$

where we now take

$$
G=e^{\beta_{1} w^{2} / 2 \rho}, \quad H_{w}=G\left(w+\frac{\alpha_{0}}{\rho^{1 / 2}} w^{2}-\frac{\beta_{0}}{\rho} w^{3}\right), \quad H(0, \rho)=0 .
$$

Then it follows that (using also equations (4.3), (4.7), (4.8))

$$
\partial_{\rho}\left(G(w, \rho) \frac{\dot{w}^{2}}{2}+H(w, \rho)\right)=G_{\rho} \frac{\dot{w}^{2}}{2}+H_{\rho}+G \dot{w}\left(F+\frac{1}{\rho^{2}} \partial_{y}^{2} w-\frac{1}{4 \rho^{2}} w\right) .
$$

Writing $H_{w}=f\left(w / \rho^{1 / 2}\right) w$ for some smooth function $f$ it follows that

$$
H=f\left(w / \rho^{1 / 2}\right) \frac{w^{2}}{2}-\rho \int_{0}^{w / \rho^{1 / 2}} f^{\prime}(s) \frac{s^{2}}{2} d s
$$

from which it is easy to see that

$$
w^{2} / 4 \leq H \leq w^{2}, \quad 1 / 4 \leq G \leq 4, \quad \text { if } \quad w^{2} \leq \mu \rho
$$

for some constant $\mu>0$ depending on $\alpha_{0}, \beta_{0}, \beta_{1}$, and

$$
H_{\rho} \lesssim \frac{1}{\rho^{3 / 2}} w^{2}|w|, \quad G_{\rho} \lesssim \frac{1}{\rho^{3 / 2}}|w|, \quad \text { if } \quad w^{2} \leq \mu \rho .
$$

Hence if we also use (4.6), then

$\partial_{\rho}\left(G(w, \rho) \frac{\dot{w}^{2}}{2}+H(w, \rho)\right) \lesssim \frac{1}{\rho^{3 / 2}}\left(\dot{w}^{2}+w^{2}\right)|w|+\left(|F|+K \rho^{\delta+3 \sigma / 2-2}+K \rho^{\delta-2}\right)|\dot{w}|$

if $w^{2} \leq \mu \rho$. This is satisfied if $C K^{2} \rho^{2 \delta} \leq \mu \rho$, i.e. if $\rho \geq \rho_{0}=\max \left\{\left(C K^{2} / \mu\right)^{1 /(1-2 \delta)}, 1\right\}$. If $\rho \leq \rho_{0}$ we use the bound $K \rho_{0}^{\delta}$, (4.4), and for $\rho \geq \rho_{0}$ we integrate the above equation to get the bound. In fact, if $E=\sqrt{G \dot{w}^{2} / 2+H}$ and we use (4.6) we get

$$
\partial_{\rho} E \leq C \frac{K \rho^{\delta}}{\rho^{3 / 2}} E+C|F|+C K \rho^{\delta+3 \sigma / 2-2} .
$$

Multiplying by the integrating factor, we get

$$
\partial_{\rho}\left(E e^{-g}\right) \leq C\left(|F|+K \rho^{\delta+3 \sigma / 2-2}\right) e^{-g},
$$

where

$$
g(\rho)=C \int_{\rho_{0}}^{\rho} K s^{\delta} s^{-3 / 2} d s \lesssim K \rho_{0}^{\delta-1 / 2} \lesssim \mu^{1 / 2}, \quad \text { if } \rho \geq \rho_{0}
$$

Hence

$$
E(\rho) \lesssim E\left(\rho_{0}\right)+\int_{\rho_{0}}^{\rho}|F(s)| d s+K,
$$


where

$$
E\left(\rho_{0}\right) \lesssim K \rho_{0}^{\delta} \lesssim K\left(1+K^{2 \delta /(1-2 \delta)}\right)
$$

Lemma 4.3 (Low frequency cubic decay estimate). Suppose that $v$ solves the equation inside the time slab $[1, T] \times \mathbb{R}$ :

$$
\partial_{\rho}^{2} v-\frac{1}{\rho^{2}} \partial_{y}^{2} v+\left(1+\frac{1}{4 \rho^{2}}\right) v-\frac{\beta_{0}}{\rho} v^{3}+\frac{\beta_{1}}{\rho} v \dot{v}^{2}=\frac{\beta(\rho y)}{\rho} v^{3}+F .
$$

Suppose that with $0<\delta<1 / 8$,

$$
K=\sup _{1 \leq \rho \leq T} \rho^{-\delta}\|(v, \dot{v})(\rho)\|_{H^{1}}<\infty .
$$

Then for $6 \delta<\sigma<1-\delta, \sigma \leq 2 / 3$,

$$
\sup _{1 \leqslant \rho \leqslant T}\|(v, \dot{v})(\rho)\|_{L^{\infty}} \lesssim K\left(1+K^{2 \delta /(1-2 \delta)}+K^{2}\right)+\int_{1}^{T}\left\|P_{\leq \rho^{\sigma}} F(\rho)\right\|_{L^{\infty}} d \rho .
$$

Proof. First, we control the high frequency part:

$$
\left\|P_{\geq \rho^{\sigma}}(v, \dot{v})\right\|_{L^{\infty}} \lesssim \rho^{-\sigma / 2}\|(v, \dot{v})\|_{H^{1}} \lesssim K,
$$

since, by our conditions on $\rho, \sigma, \sigma / 2 \geq \delta$. Applying the projection on low frequency to the equation gives with $v_{1}=P_{\leq \rho^{\sigma}} v$ :

$$
\partial_{\rho}^{2} v_{1}-\frac{1}{\rho^{2}} \partial_{y}^{2} v_{1}+\left(1+\frac{1}{4 \rho^{2}}\right) v_{1}-\frac{\beta_{0}}{\rho} v_{1}^{3}+\frac{\beta_{1}}{\rho} v_{1} \dot{v}_{1}^{2}=P_{\leq \rho^{\sigma}} F+R,
$$

where we must estimate

$$
\begin{aligned}
R=\left[\partial_{\rho}^{2}, P_{\leq \rho^{\sigma}}\right] v+\frac{1}{\rho} P_{\leq \rho^{\sigma}}\left[\beta v^{3}\right] & \\
& +\frac{\beta_{0}}{\rho}\left(P_{\leq \rho^{\sigma}} v^{3}-v_{1}^{3}\right)+\frac{\beta_{1}}{\rho}\left(P_{\leq \rho^{\sigma}} v \dot{v}^{2}-v_{1} \dot{v}_{1}^{2}\right) .
\end{aligned}
$$

Since

$$
P_{\leq \rho^{\sigma}} v(y)=\int e^{i y \xi} \chi\left(\xi / \rho^{\sigma}\right) \hat{v}(\xi) d x
$$

where $\chi \in C_{0}^{\infty}$ is 1 on $[-1,1]$ and 0 outside $[-2,2]$, we have

$$
\left[\partial_{\rho}, P_{\leq \rho^{\sigma}}\right] v(y, \rho)=-\frac{\sigma}{\rho} \int e^{i y \xi} \chi^{\prime}\left(\frac{\xi}{\rho^{\sigma}}\right) \frac{\xi}{\rho^{\sigma}} \hat{v}(\xi, \rho) d x=-\frac{\sigma}{\rho} P_{\sim \rho^{\sigma}}^{\prime} v(y, \rho),
$$

where now $\chi^{\prime}$ vanishes inside $[-1,1]$ and outside $[-2,2]$. It follows that

$$
\left\|\left[\partial_{\rho}^{2}, P_{\leq \rho^{\sigma}}\right] v\right\|_{L^{\infty}} \lesssim \frac{1}{\rho}\left(\left\|P_{\sim \rho^{\sigma}}^{\prime} \dot{v}\right\|_{L^{\infty}}+\left\|P_{\sim \rho^{\sigma}}^{\prime} v\right\|_{L^{\infty}}\right) \leq \frac{1}{\rho^{1+\sigma}}\|(v, \dot{v})(\rho)\|_{H^{1}} .
$$

Next

$$
\left\|\left(1-P_{\leq \rho^{\sigma}}\right) v^{3}\right\|_{L^{\infty}} \lesssim \frac{1}{\rho^{\sigma / 2}}\left\|v^{3}\right\|_{H^{1}} \lesssim \frac{1}{\rho^{\sigma / 2}}\|v\|_{L^{\infty}}^{2}\|v\|_{H^{1}} \lesssim \frac{1}{\rho^{\sigma / 2}}\|v\|_{H^{1}}^{3} .
$$

Similarly

$$
\left\|v^{3}-v_{1}^{3}\right\|_{L^{\infty}} \lesssim\left\|v-v_{1}\right\|_{L^{\infty}}\left(\|v\|_{L^{\infty}}+\left\|v_{1}\right\|_{L^{\infty}}\right)^{2} \lesssim \frac{1}{\rho^{\sigma / 2}}\left\|v-v_{1}\right\|_{H^{1}}\left(\|v\|_{H^{1}}+\left\|v_{1}\right\|_{H^{1}}\right)^{2} .
$$


The terms with derivatives $\dot{v}$ are estimated in a similar fashion. Note that

$$
\begin{aligned}
& \text { (4.29) }\left\|P_{\leq \rho^{\sigma}}[f g]\right\|_{L^{\infty}} \lesssim \iint_{|\xi| \leq \rho^{\sigma}}|\hat{f}(\xi-\eta)||\hat{g}(\eta)| d \eta d \xi \\
& \leq \iint_{|\xi-\eta| \leq 2 \rho^{\sigma}}|\hat{f}(\xi-\eta)||\hat{g}(\eta)| d \eta d \xi+\iint_{|\eta| \geq \rho^{\sigma}}|\hat{f}(\xi-\eta)||\hat{g}(\eta)| d \eta d \xi \\
& \quad \leq \int_{|\xi| \leq 2 \rho^{\sigma}}|\hat{f}(\xi)| d \xi \int|\hat{g}(\eta)| d \eta+\int|\hat{f}(\xi)| d \xi \int_{|\eta| \geq \rho^{\sigma}}|\hat{g}(\eta)| d \eta \\
& \leq\left[\int_{|\xi| \leq 2 \rho^{\sigma}}|\hat{f}(\xi)| d \xi\left(\int \frac{d \eta}{\langle\eta\rangle^{2}}\right)^{1 / 2}+\int|\hat{f}(\xi)| d \xi\left(\int_{|\eta| \geq \rho^{\sigma}} \frac{d \eta}{\langle\eta\rangle^{2}}\right)^{1 / 2}\right]\left(\int\langle\eta\rangle^{2}|\hat{g}(\eta)|^{2} d \eta\right)^{1 / 2}
\end{aligned}
$$

where $\langle\eta\rangle=\left(1+\eta^{2}\right)^{1 / 2}$. Since the Fourier transform of $\beta(\rho y)$ is $\widehat{\beta}(\xi / \rho) / \rho$ it follows that

$$
\int_{|\xi| \leq 2 \rho^{\sigma}}|\widehat{\beta}(\xi / \rho) / \rho| d \xi=\int_{|\xi| \leq 2 \rho^{\sigma-1}}|\widehat{\beta}(\xi)| d \xi \leq C_{\beta} \rho^{\sigma-1}
$$

Hence

$$
\left\|P_{\leq \rho^{\sigma}}\left[\left(\beta-\beta_{0}\right) v^{3}\right]\right\|_{L^{\infty}} \lesssim C\left(\rho^{\sigma-1}+\rho^{-\sigma / 2}\right)\left\|v^{3}\right\|_{H^{1}} \lesssim C \rho^{-\sigma / 2}\|v\|_{H^{1}}^{3}
$$

since $\sigma \leq 2 / 3$. It follows that

$$
\int_{1}^{T}\|R(\rho)\|_{L^{\infty}} d \rho \lesssim K^{3}
$$

Lemma 4.4 (Low frequency quadric normal-form decay estimate). Suppose that $v$ solves the equation inside the time slab $[1, T] \times \mathbb{R}$ :

$$
\partial_{\rho}^{2} v-\frac{1}{\rho^{2}} \partial_{y}^{2} v+\left(1+\frac{1}{4 \rho^{2}}\right) v-\frac{\alpha_{0}}{\rho^{1 / 2}} v^{2}-\frac{\beta_{0}}{\rho} v^{3}+\frac{\beta_{1}}{\rho} v \dot{v}^{2}=\frac{\beta(\rho y)}{\rho} v^{3} .
$$

Suppose that with $0<\delta<1 / 8$,

$$
K=\sup _{1 \leq \rho \leq T} \rho^{-\delta}\|(v, \dot{v})(\rho)\|_{H^{1}}<\infty .
$$

Then

$$
\sup _{1 \leqslant \rho \leqslant T}\|(v, \dot{v})(\rho)\|_{L^{\infty}} \lesssim K\left(1+K^{2}\right) .
$$

Proof. What is different from the previous lemma is that we now also have the term with $\alpha_{0}$ which we deal with, with a normal-form transformation. Let

$$
w=\frac{\alpha_{0}}{3 \rho^{1 / 2}}\left(v_{4}^{2}+2 \dot{v}_{4}^{2}\right), \quad v_{4}=P_{\leq 4 \lambda} v, \quad \lambda=\rho^{\sigma} .
$$

Then

$$
\begin{aligned}
& \left(\partial_{\rho}^{2}+1\right) w=\frac{\alpha_{0}}{3 \rho^{1 / 2}}\left(2 v_{4} \ddot{v}_{4}+2 \dot{v}_{4}^{2}+4 \dot{v}_{4} \dddot{v}_{4}+4 \ddot{v}_{4}^{2}+v_{4}^{2}+2 \dot{v}_{4}^{2}\right)+O\left(\frac{1}{\rho^{3 / 2}}\right) \\
& =\frac{\alpha_{0}}{3 \rho^{1 / 2}}\left(4 \dot{v}_{4}\left(\dddot{v}_{4}+\dot{v}_{4}\right)+4\left(\ddot{v}_{4}+v_{4}\right)^{2}+3 v_{4}^{2}-6\left(\ddot{v}_{4}+v_{4}\right) v_{4}\right)+O\left(\frac{1}{\rho^{3 / 2}}\right),
\end{aligned}
$$


so with $\square_{1}=\partial_{\rho}^{2}-\rho^{-2} \partial_{y}^{2}+1$,

$$
\begin{aligned}
& \text { 4.38) } \quad \square_{1} w-\frac{\alpha_{0}}{\rho^{1 / 2}} v_{4}^{2}-\frac{\alpha_{0}^{2}}{\rho}\left(\frac{8}{3} v_{4} \dot{v}_{4}^{2}-2 v_{4}^{3}\right) \\
& =F_{1}=\frac{\alpha_{0}}{3 \rho^{1 / 2}}\left(4 \dot{v}_{4}\left(\square_{1} \dot{v}_{4}-2 \frac{\alpha_{0}}{\rho^{1 / 2}} v_{4} \dot{v}_{4}\right)-6 v_{4}\left(\square_{1} v_{4}-\frac{\alpha_{0}}{\rho^{1 / 2}} v_{4}^{2}\right)+4\left(\square_{1} v_{4}\right)^{2}\right. \\
& \left.+\left(-8 \square_{1} v_{4}+4\left(v_{4}-\frac{1}{\rho^{2}} \partial_{y}^{2} v_{4}\right)\right) \frac{1}{\rho^{2}} \partial_{y}^{2} v_{4}+2\left(\frac{1}{\rho} \partial_{y} v_{4}\right)^{2}+4\left(\frac{1}{\rho} \partial_{y} \dot{v}_{4}\right)^{2}\right)+O\left(\frac{1}{\rho^{3 / 2}}\right) .
\end{aligned}
$$

Here

$$
\square_{1} v_{4}=\ddot{v}_{4}+v_{4}-\frac{1}{\rho^{2}} \partial_{y}^{2} v_{4}=\left[\partial_{\rho}^{2}, P_{\leq 4 \rho^{\sigma}}\right] v+\frac{\alpha_{0}}{\rho^{1 / 2}} P_{\leq 4 \lambda} v^{2}+O\left(\frac{1}{\rho}\right)
$$

and

$$
P_{\leq 4 \lambda}(u v)-u_{4} v_{4}=\left(\left(u-u_{4}\right) v+u_{4}\left(v-v_{4}\right)\right)+P_{\geq 4 \lambda}(u v),
$$

SO

$$
\left|P_{\leq 4 \lambda}(u v)-u_{4} v_{4}\right| \lesssim \frac{1}{\lambda^{1 / 2}}\|u\|_{H^{1}}\|v\|_{H^{1}}
$$

It follows that

$$
\left|F_{1}\right| \lesssim \frac{K^{2}}{\rho^{1+1 / 4}}
$$

We have

$$
\begin{aligned}
\left(\partial_{\rho}^{2}-\frac{1}{\rho^{2}} \partial_{y}^{2} v+1+\frac{1}{4 \rho^{2}}\right)(v-w) & +\frac{\alpha_{0}^{2}}{\rho}\left(\frac{8}{3} v_{4} \dot{v}_{4}^{2}-2 v_{4}^{3}\right)-\frac{\beta_{0}}{\rho} v^{3}+\frac{\beta_{1}}{\rho} v \dot{v}^{2} \\
= & \frac{\alpha_{0}}{\rho^{1 / 2}}\left(v^{2}-v_{4}^{2}\right)+\frac{\beta(\rho y)}{\rho} v^{3}-\frac{1}{4 \rho^{2}} w-F_{1} .
\end{aligned}
$$

In the terms in the top row we can first replace $v_{4}$ by $v$ with an error $\rho^{-1-\sigma / 2+2 \delta}$ and then replace $v$ by $v-w$ with an error $\rho^{-1-1 / 2+2 \delta}$, so with $\left|F_{2}\right| \lesssim \rho^{-1-1 / 4}$ we have

$$
\begin{aligned}
&\left(\partial_{\rho}^{2}-\frac{1}{\rho^{2}} \partial_{y}^{2} v+1+\frac{1}{4 \rho^{2}}\right)(v-w)+\frac{8 \alpha_{0}^{2} / 3-\beta_{0}}{\rho}(v-w)^{3}+\frac{\beta_{1}+2 \alpha_{0}^{2}}{\rho}(v-w)(\dot{v}-\dot{w})^{2} \\
&=\frac{\alpha_{0}}{\rho^{1 / 2}}\left(v^{2}-v_{4}^{2}\right)+\frac{\beta(\rho y)}{\rho}(v-w)^{3}-\frac{1}{4 \rho^{2}} w-F_{1}+F_{2} .
\end{aligned}
$$

We are now in position to apply the previous lemma. The only term that remains to be controlled is

$$
\left|P_{\leq \lambda}\left(v^{2}-v_{4}^{2}\right)\right| \lesssim\left|P_{\leq \lambda}\left(v-v_{4}\right)^{2}\right|+\left|P_{\leq \lambda}\left(\left(v-v_{4}\right) P_{\geq 3 \lambda} v_{4}\right)\right| .
$$

In either case we have a product of factors $u w$ each of which has frequencies $\geq 2 \lambda$, say, so

$$
\begin{aligned}
&\left|P_{\leq \lambda}(u w)\right| \leq\|u w\|_{L^{2}}^{1 / 2}\|u w\|_{H^{1}}^{1 / 2} \\
& \lesssim\left(\|u\|_{L^{\infty}}+\|w\|_{L^{\infty}}\right)\left(\|u\|_{L^{2}}+\|w\|_{L^{2}}\right)^{1 / 2}\left(\|u\|_{H^{1}}+\|w\|_{H^{1}}\right)^{1 / 2} \\
& \quad \lesssim\left(\|u\|_{L^{2}}+\|w\|_{L^{2}}\right)\left(\|u\|_{H^{1}}+\|w\|_{H^{1}}\right) \lesssim \frac{1}{\lambda}\left(\|u\|_{H^{1}}+\|w\|_{H^{1}}\right)^{2} .
\end{aligned}
$$


The proof follows from this. Note that the critical thing here was that for each of the factors $\left(v-v_{4}\right) v_{4}$ only frequencies higher than $3 \lambda$ entered because of the projection $P_{\leq \lambda}$.

Remark 4.5. Note that the region with frequencies of $\beta(\rho y)$ less than $\lambda \leq \rho^{1 / 2}$ is easy to deal with. In fact, with $\chi(s)$ a cutoff function supported when $|s| \leq 2$ we have

$$
P_{\leq \rho^{\sigma}} \beta(\rho y)=\int e^{i y \rho \zeta} \chi\left(\zeta \rho^{1-\sigma}\right) \hat{\beta}(\zeta) d \zeta
$$

It follows that

$$
\left|\partial_{y}^{k} P_{\leq \rho^{\sigma}} \beta(\rho y)\right| \lesssim \rho^{(k+1) \sigma-1}
$$

and

$$
\left\|\partial_{y}^{k} P_{\leq \rho^{\sigma}} \beta(\rho y)\right\|_{L_{y}^{2}} \lesssim \rho^{(k+1) \sigma-3 / 2} .
$$

It follows that these are bounded for $k \leq 1$ as long as $\sigma \leq 1 / 2$ or $\sigma \leq 3 / 4$, respectively. See also the proof of Lemmas 4.3 and 4.4 .

\section{Semi-Classical operators}

All of the multilinear operators we consider in this paper are defined for fixed time (but depend on time) and in general are nonlocal in the spatial variable. Because the derivative $\rho^{-1} \partial_{y}$ has a semi-classical nature as $\rho^{-1} \rightarrow 0$, we will define all of our operators semi-classically with respect to the derivative:

$$
D_{y}=\frac{1}{i \rho} \partial_{y} \text {. }
$$

This will turn out to be very convenient in calculations, but one needs to keep in mind that there is a nontrivial commutator with $\partial_{\rho}$ :

$$
\left[\partial_{\rho}, D_{y}\right]=-\frac{1}{\rho} D_{y} .
$$

This will lead to certain error terms in the sequel, but they are asymptotically mild given that they contain an extra power of decay in $\rho$. In terms of this notation, the equation (2.5) becomes

$$
\left(\partial_{\rho}^{2}+D_{y}^{2}+\left(1+\frac{1}{4 \rho^{2}}\right)\right) v=\frac{\alpha_{0}}{\rho^{\frac{1}{2}}} v^{2}+\frac{\beta(\rho y)}{\rho} v^{3}+\frac{\beta_{0}}{\rho} v^{3}+\frac{1}{\rho} \mathcal{R}_{\beta}(\rho, y) v^{3} .
$$

Also associated with this notation, one has the semi-classical version of the Fourier transform (3.1):

$$
\mathcal{F}_{\rho}(v)(\xi)=\widetilde{v}(\xi)=\rho \int_{\mathbb{R}} e^{-i \rho \xi y} v(y) d y=\rho \hat{v}(\rho \xi), \quad \hat{v}(\xi)=\int e^{i x \xi} v(x) d x .
$$

This definition gives $\widetilde{D_{y} v}=\xi \widetilde{v}$. The inverse of the transformation is of course

$$
v(y)=\mathcal{F}_{\rho}^{-1}(\widetilde{v})(y)=\frac{1}{2 \pi} \int_{\mathbb{R}} e^{i \rho y \xi} \widetilde{v}(\xi) d \xi .
$$

It is important to keep in mind, although we suppress it with the $\widetilde{v}$ notation, that our semi-classical Fourier transform is time dependent, and one has the commutator relation

$$
\left[\partial_{\rho}, \mathcal{F}_{\rho}\right]=-i \mathcal{F}_{\rho} y D_{y} .
$$

In the present work, it will turn out that expressions of this form will not play a role because all of our operators will be translation invariant with respect to $y$. 
We now define the type of bilinear $\Psi D O$ we are working with here. Given a "symbol" $\widehat{b}(\xi, \eta)$, we define its associated operator:

$$
\begin{aligned}
B\left({ }^{1} D_{y},{ }^{2} D_{y}\right)(u, v) & =\frac{1}{4 \pi^{2}} \iint b(\xi, \eta) e^{i \rho y(\xi+\eta)} \widetilde{u}(\xi) \cdot \widetilde{v}(\eta) d \xi d \eta \\
& =\frac{1}{4 \pi^{2}} \iint b(\xi / \rho, \eta / \rho) e^{i y(\xi+\eta)} \widehat{u}(\xi) \cdot \widehat{v}(\eta) d \xi d \eta \\
& \equiv B(u, v) .
\end{aligned}
$$

We say that $B$ is a bilinear $\Psi D O$ if its symbol obeys the uniform bounds:

$$
\sum_{k+\ell \leq N} \sup _{\xi, \eta}(1+|\xi|)^{k}(1+|\eta|)^{\ell}\left|\partial_{\xi}^{k} \partial_{\eta}^{\ell} b(\xi, \eta)\right| \leq C_{N} .
$$

A typical such expression would be something like $b(\xi, \eta)=\frac{q(\xi, \eta)}{p(\xi, \eta)}$ for polynomials $p$ and $q$, with $p$ nonvanishing and of degree greater that or equal to the degree of $q$. To further our computations, we record here the effect of differentiating the expressions $K(u, v)$ :

Lemma 5.1 (Bilinear $\Psi$ DO calculus). Let $K$ be an operator as defined on (5.4). Then one has the following identities:

$$
D_{y} B\left({ }^{1} D_{y},{ }^{2} D_{y}\right)(u, v)=B\left({ }^{1} D_{y},{ }^{2} D_{y}\right)\left(D_{y} u, v\right)+B\left({ }^{1} D_{y},{ }^{2} D_{y}\right)\left(u, D_{y} v\right)
$$

and

$$
\begin{array}{r}
\partial_{\rho} B\left({ }^{1} D_{y},{ }^{2} D_{y}\right)(u, v)=B\left({ }^{1} D_{y},{ }^{2} D_{y}\right)\left(\partial_{\rho} u, v\right)+B\left({ }^{1} D_{y},{ }^{2} D_{y}\right)\left(u, \partial_{\rho} v\right) \\
-\rho^{-1} \partial_{1} B\left({ }^{1} D_{y},{ }^{2} D_{y}\right)\left(D_{y} u, v\right)-\rho^{-1} \partial_{2} B\left({ }^{1} D_{y},{ }^{2} D_{y}\right)\left(u, D_{y} v\right) .
\end{array}
$$

Here $\partial_{1} B$ and $\partial_{2} B$ have symbols $\partial_{\xi} b$ and $\partial_{\eta} b$ respectively.

Proof of the identities (5.6) -(5.7). We only prove the second identity (5.7), as the first is immediate. By rescaling into the time independent Fourier variables given by (3.1), (5.4) may be rewritten as

$$
B\left({ }^{1} D_{y},{ }^{2} D_{y}\right)(u, v)=\frac{1}{4 \pi^{2}} \iint b\left(\rho^{-1} \xi, \rho^{-1} \Theta\right) e^{i y(\xi+\Theta)} \widehat{u}(\xi) \cdot \widehat{v}(\Theta) d \xi d \Theta .
$$

Taking a $\partial_{\rho}$ derivative of this last line and then rescaling back to the semi-classical variables $\xi, \eta$, we see that

$$
\begin{aligned}
& \partial_{\rho} B\left({ }^{1} D_{y},{ }^{2} D_{y}\right)(u, v)=-\frac{1}{4 \pi^{2}} \iint \frac{\xi}{\rho} \partial_{\xi} b(\xi, \eta) e^{i \rho y(\xi+\eta)} \widetilde{u}(\xi) \cdot \widetilde{v}(\eta) d \xi d \eta \\
&-\frac{1}{4 \pi^{2}} \iint \frac{\eta}{\rho} \partial_{\eta} b(\xi, \eta) e^{i \rho y(\xi+\eta)} \widetilde{u}(\xi) \cdot \widetilde{v}(\eta) d \xi d \eta .
\end{aligned}
$$

The proof is finished with an application of the identity $\widetilde{D_{y} v}=\xi \widetilde{v}$.

Finally, we end this section by listing and proving a set of estimates that will allow us to control expressions involving operators of the form $K(u, v)$. These are:

Proposition 5.2 (Estimates for $\Psi D O)$. Let $b(\xi, \eta)$ be a symbol which obeys the uniform bounds (5.5), for $N \geq 2$. Then if $B(u, v)$ is the operator defined on (5.4), 
one has the uniform bounds:

$$
\begin{aligned}
\|B(u, v)\|_{L^{p}} & \lesssim\|B\|\|u\|_{L^{p}} \cdot\|v\|_{B_{\rho}^{\infty}}, \quad 1 \leq p \leq \infty \\
\|B(u, v)\|_{H^{1}} & \lesssim\|B\|\left(\|u\|_{H^{1}} \cdot\|v\|_{B_{\rho}^{\infty}}+\|u\|_{B_{\rho}^{\infty}} \cdot\|v\|_{H^{1}}\right) \\
\|B(u, v)\|_{B_{\rho}^{\infty}} & \lesssim\|B\|\|u\|_{B_{\rho}^{\infty}} \cdot\|v\|_{B_{\rho}^{\infty}} .
\end{aligned}
$$

Here

$$
\|B\| \lesssim\|B\|_{1,1}\left(1+\ln \left(\|B\|_{2,2} /\|B\|_{1,1}\right)\right)^{2}
$$

where

$$
\|B\|_{M, N}=\max _{k, \ell \geq 0} \sum_{m \leq M, n \leq N} \iint_{D_{k, \ell}}(1+|\xi|)^{m-1}(1+|\eta|)^{n-1}\left|\partial_{\xi}^{m} \partial_{\eta}^{n} b(\xi, \eta)\right| d \xi d \eta
$$

and $D_{0,0}=\{|\xi|,|\eta| \leq 4\}$ and $D_{k, \ell}=\left\{2^{-1} \leq|\xi| / 2^{k},|\eta| / 2^{\ell} \leq 2\right\}$.

The proof of the proposition will follow from dyadic decomposition and integration by parts on the Fourier transform side; it uses the following well known estimate (proven by duality):

Lemma 5.3. For $1 \leq p \leq \infty$ we have

$$
\left\|\iint K\left(y ;, x_{1}, x_{2}\right) u\left(x_{1}\right) v\left(x_{2}\right) d x_{1} d x_{2}\right\|_{L^{p}(y)} \leq C(K)\|u\|_{L^{p}} \cdot\|v\|_{L^{\infty}}
$$

$$
C(K)=\sup _{y}\left\|K\left(y ; x_{1}, x_{2}\right)\right\|_{L_{x_{1}, x_{2}}^{1}}+\sup _{x_{1}}\left\|K\left(y ; x_{1}, x_{2}\right)\right\|_{L_{y, x_{2}}^{1}}<\infty .
$$

Proof of the estimate (5.8). We begin with the proof of (5.8). An important property of the translation independent bilinear operators $B(u, v)$ we are working with is that they obey the same frequency combination rules as ordinary products. Thus, one may always decompose them via "trichotomy". This follows at once from taking the semi-classical Fourier transform of the RHS of (5.4), which gives (note that the usual FT (3.1) is just a rescaling of this)

$$
\rho \int_{\mathbb{R}} e^{-i \rho \zeta y} B(u, v)(y) d y=\frac{1}{2 \pi} \iint_{\zeta=\xi+\eta} b(\xi, \eta) \widetilde{u}(\xi) \cdot \widetilde{v}(\eta) d \xi d \eta .
$$

Because the operator $B(u, v)$ is semi-classical, it also has a preferred scale. Therefore, we first make a bulk decomposition:

$$
B(u, v)=P_{\lesssim \rho} B(u, v)+P_{\gtrsim \rho} B(u, v) .
$$

We will prove (5.8) for each of these terms separately, beginning with the first. This term may again be decomposed as follows:

$$
P_{\lesssim \rho} B(u, v)=P_{\lesssim \rho} B\left(P_{\lesssim \rho} u, P_{\lesssim \rho} v\right)+\sum_{\mu_{1} \sim \mu_{2} \gtrsim \rho} P_{\lesssim \rho} B\left(P_{\mu_{1}} u, P_{\mu_{2}} v\right) .
$$

Note that here the last sum is over all $\left|\mu_{1}+\mu_{2}\right| \leq \rho$, but since also $\left|\mu_{1}\right|>\rho$ and $\left|\mu_{2}\right|>\rho$ and the sum is over dyadic $2^{k}$, there is only finitely many $\mu_{1}$ satisfying this for each $\mu_{2}$. Our aim is now to prove the two frequency localized estimates:

$$
\begin{aligned}
\left\|B\left(P_{\lesssim \rho} u, P_{\lesssim \rho} v\right)\right\|_{L^{p}} & \lesssim\|B\|\|u\|_{L^{p}} \cdot\|v\|_{L^{\infty}}, \\
\left\|B\left(P_{\mu_{1}} u, P_{\mu_{2}} v\right)\right\|_{L^{p}} & \lesssim\|B\|\left\|P_{\mu_{1}} u\right\|_{L^{p}} \cdot\left\|P_{\mu_{2}} v\right\|_{L^{\infty}} .
\end{aligned}
$$


By summing over all $\mu_{1} \sim \mu_{2} \gtrsim \rho$ in the second estimate we see that (5.17) implies

$$
\sum_{\mu_{1} \sim \mu_{2} \gtrsim \rho}\left\|B\left(P_{\mu_{1}} u, P_{\mu_{2}} v\right)\right\|_{L^{p}} \lesssim \sup _{\mu \geq \rho}\left\|P_{\mu} u\right\|_{L^{p}} \cdot \sum_{\mu \geq \rho}\left\|P_{\mu} v\right\|_{L^{\infty}} \lesssim\|u\|_{L^{p}} \cdot\|v\|_{B_{\rho}^{\infty}} .
$$

This, together with (5.16), will establish (5.8) for the first term on the RHS of (5.15).

We now try to prove (5.16) (5.17). Both of these will result from a similar argument. The goal is to show that in each case the associated integral kernel $K\left(y ; x_{1}, x_{2}\right)$ enjoys a sort of uniform bilinear $L^{1}$ property.

Therefore, we only need to recover the conditions (5.14) for the kernels of the bilinear operators $B\left(P_{\lesssim \rho} u, P_{\lesssim \rho} v\right)$ and $B\left(P_{\mu_{1}} u, P_{\mu_{2}} v\right)$. The symbols are respectively given by

$$
b_{\lesssim \rho, \lesssim \rho}(\xi, \eta)=b(\xi, \eta) \chi(\xi) \chi(\eta), \quad b_{\mu_{1}, \mu_{2}}(\xi, \eta)=b(\xi, \eta) p_{1}\left(\rho \mu_{1}^{-1} \xi\right) p_{1}\left(\rho \mu_{2}^{-1} \eta\right) .
$$

Here $\chi \equiv 1$ in a neighborhood of 0 , and $p_{1}$ is an annular cutoff of the form used to define $P_{\lambda}$ for $1<\lambda$. To obtain the desired bounds, in both cases we compute the physical space kernel from the symbol

$$
K\left(y ; x_{1}, x_{2}\right)=\frac{1}{4 \pi} \rho^{2} \iint b(\xi, \eta) e^{i \rho \xi\left(y-x_{1}\right)} e^{i \rho \eta\left(y-x_{2}\right)} d \xi d \eta .
$$

Multiple integration by parts then shows that for any $M, N \geq 0$,

$$
\left|K\left(y ; x_{1}, x_{2}\right)\right| \lesssim \frac{\rho^{2}}{\left(\rho\left|y-x_{1}\right|\right)^{M}\left(\rho\left|y-x_{2}\right|\right)^{N}} \iint\left|\partial_{\xi}^{M} \partial_{\eta}^{M} b(\xi, \eta)\right| d \xi d \eta .
$$

Here

$$
\iint\left|\partial_{\xi}^{M} \partial_{\eta}^{M} b_{\lesssim \rho, \lesssim \rho}(\xi, \eta)\right| d \xi d \eta \lesssim\|B\|_{M, N}
$$

and

$$
\iint\left|\partial_{\xi}^{M} \partial_{\eta}^{M} b_{\mu_{1}, \mu_{2}}(\xi, \eta)\right| d \xi d \eta \lesssim\|B\|_{M, N} \frac{\rho^{M+N}}{\mu_{1}^{M} \mu_{2}^{N}}
$$

Hence one has the bounds

$$
\begin{aligned}
\left|K_{\lesssim \rho, \lesssim \rho}\left(y ; x_{1}, x_{2}\right)\right| & \lesssim \frac{\|B\|_{M, N} \rho^{2}}{\left(1+\rho\left|y-x_{1}\right|\right)^{M}\left(1+\rho\left|y-x_{2}\right|\right)^{N}}, \\
\left|K_{\mu_{1}, \mu_{2}}\left(y ; x_{1}, x_{2}\right)\right| & \lesssim \frac{\|B\|_{M, N} \mu_{1} \mu_{2}}{\left(1+\mu_{1}\left|y-x_{1}\right|\right)^{M}\left(1+\mu_{2}\left|y-x_{2}\right|\right)^{N}} .
\end{aligned}
$$

These easily imply (5.14) with a constant as in (5.11) in both cases. In fact, for the first one when $\rho\left|y-x_{1}\right| \leq\|B\|_{2,2} /\|B\|_{1,1}$ and $\rho\left|y-x_{2}\right| \leq\|B\|_{2,2} /\|B\|_{1,1}$ we use the inequality for $M=N=1$, and in the complement we use the inequality for $M=N=2$.

To finish, we need to establish (5.8) for the second term on the RHS of (5.15). This will result from decompositions and estimates that are almost identical to what we have just done. We begin by decomposing:

$$
\begin{aligned}
P_{\gtrsim \rho} B(u, v)=\sum_{\lambda \gtrsim \rho} P_{\lambda} B\left(P_{\lambda} u, P_{\ll \lambda} v\right)+ & \sum_{\lambda \gtrsim \rho} P_{\lambda} B\left(P_{\ll \lambda} u, P_{\lambda} v\right) \\
& +\sum_{\substack{\lambda \gtrsim \rho \\
\mu_{1} \sim \mu_{2} \gtrsim \lambda}} P_{\lambda} B\left(P_{\mu_{1}} u, P_{\mu_{2}} v\right) .
\end{aligned}
$$


For the last term we have

$$
\begin{aligned}
& \sum_{\mu_{1} \sim \mu_{2} \gtrsim \lambda \gtrsim \rho}\left\|P_{\lambda} B\left(P_{\mu_{1}} u, P_{\mu_{2}} v\right)\right\|_{L^{p}} \lesssim \sum_{\mu_{1} \sim \mu_{2} \gtrsim \rho} \sum_{\mu_{2} \gtrsim \lambda \gtrsim \rho}\left\|B\left(P_{\mu_{1}} u, P_{\mu_{2}} v\right)\right\|_{L^{p}} \\
& \lesssim\|B\| \sum_{\mu_{1} \sim \mu_{2} \geq \rho} \ln \left|\mu_{2} / \rho\right|\left\|P_{\mu_{1}} u\right\|_{L^{p}}\left\|P_{\mu_{2}} v\right\|_{L^{\infty}} \lesssim\|B\|\|u\|_{L^{p}} \cdot\|v\|_{B_{\rho}^{\infty}} .
\end{aligned}
$$

By using the Plancherel theorem and a bit of dyadic summation (e.g. the dyadic convolution version of Young's inequality), it suffices to show the two estimates:

$$
\begin{aligned}
\left\|B\left(P_{\lambda} u, P_{\ll \lambda} v\right)\right\|_{L^{p}} & \lesssim\|B\|\left\|P_{\lambda} u\right\|_{L^{p}} \cdot\|v\|_{B_{\rho}^{\infty}}, \\
\left\|B\left(P_{\ll \lambda} u, P_{\lambda} v\right)\right\|_{L^{p}} & \lesssim\|B\|\|u\|_{L^{p}} \cdot \ln |\lambda / \rho|\left\|P_{\lambda} v\right\|_{L^{\infty}} .
\end{aligned}
$$

The proof of the first estimate (5.23) is also very similar to work already done. Using the $\ell^{1}$ Besov structure in the definition of the space $B_{\rho}^{\infty}$ for frequencies $\rho \leqslant \mu_{2}$, we may reduce the proof of this estimate to showing the two bounds:

$$
\begin{aligned}
\left\|B\left(P_{\lambda} u, P_{\lesssim_{\rho}} v\right)\right\|_{L^{p}} & \lesssim\|B\|\left\|P_{\lambda} u\right\|_{L^{p}} \cdot\|v\|_{L^{\infty}}, \\
\left\|B\left(P_{\lambda} u, P_{\mu_{2}} v\right)\right\|_{L^{p}} & \lesssim\|B\|\left\|P_{\lambda} u\right\|_{L^{p}} \cdot\left\|P_{\mu_{2}} v\right\|_{L^{\infty}}, \quad \rho \leqslant \mu_{2} .
\end{aligned}
$$

The proof of both of these estimates boils down to showing that the kernels of these two bilinear operators obey the bounds (5.20)-(5.21). This in turn comes from integrating by parts in the formula (5.18) to produce estimates of the form (5.20) -(5.21). We leave the details to the reader.

Finally, we need to establish (5.24). This bound may in turn be derived from the two estimates:

$$
\begin{aligned}
\left\|B\left(P_{\ll \rho} u, P_{\lambda} v\right)\right\|_{L^{p}} & \lesssim\|B\|\|u\|_{L^{p}} \cdot\left\|P_{\lambda} v\right\|_{L^{\infty}}, \\
\left\|B\left(P_{\mu_{1}} u, P_{\lambda} v\right)\right\|_{L^{p}} & \lesssim\|B\|\left\|P_{\mu_{1}} u\right\|_{L^{p}} \cdot\left\|P_{\lambda} v\right\|_{L^{\infty}}, \quad
\end{aligned}
$$

Both of these bounds result from kernel estimates similar to (5.20)-(5.21) and are left to the reader. Notice that the second estimate may be summed over all $\rho \leqslant \mu_{1} \leqslant \lambda$ at a cost of $\ln |\lambda / \rho| \sup _{\rho \lesssim \mu_{1} \lesssim \lambda}\left\|P_{\mu_{1}} u\right\|_{L^{2}}$. This gives (5.24). The details are again left to the reader. This completes our proof of the estimate (5.8). Next, note that (5.9) follows from (5.8) and (5.7). Finally we need to prove (5.10): The proof is similar to the proof of (5.8); we need to control an extra log factor $\ln \left|\mu_{2} / \rho\right|$ on the right hand side of (5.22) and a similar $\ln |\lambda / \rho|$ factor on (5.25). The result now follows from the definition of the $B_{\rho}^{\infty}$ norm.

Lemma 5.4. Let $b(\xi, \eta)$ be a symbol which obeys the uniform bounds (5.5), for $N \geq 2$, and suppose that $b(\xi, \eta)$ vanishes to second order at the origin $(0,0)$. Then if $B(u, v)$ is the operator defined on (5.4), one has the uniform bounds

$$
\left\|P_{\leq \rho^{\sigma}} B(u, v)\right\|_{B_{\rho}^{\infty}} \lesssim \rho^{-\sigma}\left(\|u\|_{B_{\rho}^{\infty}}+\|u\|_{H^{1}}\right) \cdot\left(\|v\|_{B_{\rho}^{\infty}}+\|v\|_{H^{1}}\right)
$$

for $\sigma<2 / 3$.

Proof. With the projection we now have the kernel

$$
\hat{R}(\xi, \eta)=\chi\left((\xi+\eta) \rho^{1-\sigma}\right) b(\xi, \eta) .
$$

Since $|\xi+\eta|<2 \rho^{\sigma-1}$ in the support it follows that either both $|\xi| \leq 4 \rho^{\sigma-1}$ and $|\eta| \leq 4 \rho^{\sigma-1}$ or both $|\xi| \geq 2 \rho^{\sigma-1}$ and $|\eta| \geq 2 \rho^{\sigma-1}$. Hence we have to consider two operators with kernels:

$$
\hat{R}_{1}(\xi, \eta)=\chi\left((\xi+\eta) \rho^{1-\sigma}\right) b(\xi, \eta) \chi\left(\xi \rho^{1-\sigma} / 8\right) \chi\left(\eta \rho^{1-\sigma} / 8\right)
$$


and

$$
\hat{R}_{2}(\xi, \eta)=\chi\left((\xi+\eta) \rho^{1-\sigma}\right) b(\xi, \eta)\left(1-\chi\left(\xi \rho^{1-\sigma}\right)\right)\left(1-\chi\left(\eta \rho^{1-\sigma}\right)\right) .
$$

Then its easy to see that

$$
\left\|R_{1}\right\|_{2,2} \lesssim 1, \quad\left\|R_{1}\right\|_{1,1} \lesssim \rho^{-2(1-\sigma)},
$$

and therefore $\left\|R_{1}\right\| \lesssim \rho^{-\sigma}$, if $\sigma<2 / 3$. For the last operator we simply have

$$
\left\|P_{\leq \rho^{\sigma}} B\left(P_{\geq \rho^{\sigma}} u, P_{\geq \rho^{\sigma}} v\right)\right\|_{B_{\rho}^{\infty}} \lesssim \rho^{-\sigma}\|u\|_{H^{1}}\|v\|_{H^{1}},
$$

by the previous proposition and (3.9).

\section{QUADRATIC NORMAL FORMS}

In this section, we will construct bilinear operators which can be used to control the quadratic term on the RHS of (5.1). This construction follows the classical normal-forms calculation in Shatah's seminal paper [Sh, albeit adapted to the semi-classical setup of the previous section.

Suppose that $u$ and $v$ solve the following Klein-Gordon equations:

$$
\begin{aligned}
& \left(\partial_{\rho}^{2}+D_{y}^{2}+1\right) u=F, \\
& \left(\partial_{\rho}^{2}+D_{y}^{2}+1\right) v=G .
\end{aligned}
$$

We wish to understand the nature of the solution $w$ to the equation

$$
\left(\partial_{\rho}^{2}+D_{y}^{2}+1\right) w=\frac{\alpha_{0}}{\rho^{\frac{1}{2}}} u \cdot v
$$

In particular, we are trying to obtain estimates on the LHS of the source term bound (2.14) without having to directly put the RHS of (6.1) into the space $\mathcal{S}_{\delta}[1, T]$ with uniform bounds (assuming of course that $F$ and $G$ obey reasonable estimates). To do this, we attempt to construct the solution as a power series $w=w_{0}+w_{1}+\ldots$ where the first two terms are defined by

$$
\left(\partial_{\rho}^{2}+D_{y}^{2}+1\right) w_{0}=0, \quad w_{1}=Q\left(u, v ; \partial_{\rho} u, \partial_{\rho} v\right),
$$

where $Q$ is some appropriately chosen bilinear expression. We will take these to be of the form

$$
w_{1}=\frac{1}{\rho^{\frac{1}{2}}} B_{1}(u, v)+\frac{1}{\rho^{\frac{1}{2}}} B_{2}(\dot{u}, \dot{v}),
$$

where the operators are bilinear $\Psi \mathrm{DO}$ as defined on (5.4). Here we are using the shorthand $\partial_{\rho} u=\dot{u}$, and similarly for $\dot{v}$. What we are expecting to see is the following cancellation:

$$
\left(\partial_{\rho}^{2}+D_{y}^{2}+1\right) w_{1}-\frac{\alpha_{0}}{\rho^{\frac{1}{2}}} u \cdot v=\{\text { terms which decay better }\},
$$

where the RHS is an expression which can be put into the source term space $\mathcal{S}_{\delta}[1, T]$ (assuming a reasonable form for $F$ and $G$ ). To uncover all of this, we need to do some calculations. 


\subsection{Kernel calculations for the general case.}

Lemma 6.1. Suppose that $L u=F$ and $L v=G$, where $L=\partial_{\rho}^{2}+D_{y}^{2}+1$ and set

$$
w_{1}=\frac{1}{\rho^{\frac{1}{2}}} B_{1}(u, v)+\frac{1}{\rho^{\frac{1}{2}}} B_{2}(\dot{u}, \dot{v}) .
$$

Then

$$
\begin{aligned}
& L w_{1}=\frac{1}{\rho^{\frac{1}{2}}}\left[-B_{1}(u, v)+2 B_{1}(\dot{u}, \dot{v})-B_{2}(\dot{u}, \dot{v})\right. \\
& \left.+2 B_{2}\left(\left(D_{y}^{2}+1\right) u,\left(D_{y}^{2}+1\right) v\right)+2 B_{1}\left(D_{y} u, D_{y} v\right)+2 B_{2}\left(D_{y} \dot{u}, D_{y} \dot{v}\right)\right] \\
& +\frac{1}{\rho^{\frac{1}{2}}}\left[B_{1}(F, v)+B_{1}(u, G)+2 B_{2}(F, G)+B_{2}(\dot{F}, \dot{v})+B_{2}(\dot{u}, \dot{G})\right. \\
& \left.\quad-2 B_{2}\left(\left(D_{y}^{2}+1\right) u, G\right)-2 B_{2}\left(F,\left(D_{y}^{2}+1\right) v\right)\right]+\mathcal{C}_{1}+\mathcal{C}_{2}+\mathcal{C}_{3},
\end{aligned}
$$

where the commutator terms are

$$
\begin{gathered}
\mathcal{C}_{1}=\partial_{\rho}^{2}\left(\frac{1}{\rho^{1 / 2}} B_{1}(u, v)\right)-\frac{1}{\rho^{1 / 2}} \sum_{\ell=0}^{2}\left(\begin{array}{l}
2 \\
\ell
\end{array}\right) B_{1}\left(\partial_{\rho}^{\ell} u, \partial_{\rho}^{2-\ell} v\right), \\
\mathcal{C}_{2}=\partial_{\rho}^{2}\left(\frac{1}{\rho^{1 / 2}} B_{2}(\dot{u}, \dot{v})\right)-\frac{1}{\rho^{1 / 2}} \sum_{\ell=0}^{2}\left(\begin{array}{l}
2 \\
\ell
\end{array}\right) B_{2}\left(\partial_{\rho}^{\ell} \dot{u}, \partial_{\rho}^{2-\ell} \dot{v}\right),
\end{gathered}
$$

and

$$
\mathcal{C}_{3}=D_{y}^{2}\left(\frac{1}{\rho^{1 / 2}} B_{2}(\dot{u}, \dot{v})\right)-\frac{1}{\rho^{1 / 2}}\left(2 B_{2}\left(D_{y} \dot{u}, D_{y} \dot{v}\right)+B_{2}\left(\partial_{\rho} D_{y}^{2} u, \dot{v}\right)+B_{2}\left(\dot{u}, \partial_{\rho} D_{y}^{2} v\right)\right) .
$$

The proof of the lemma follows from using the commutator formulas and then replacing $\ddot{u}=F-\left(D_{y}^{2}+1\right) u$ and $\ddot{v}=G-\left(D_{y}^{2}+1\right) v$.

The primary term which is given explicitly on the RHS of (6.4) must be set to cancel with the quadratic interaction on the RHS of (6.1). We write this as a diagonal $2 \times 2$ system in the pair of variables $(u, v)$ and $(\dot{u}, \dot{v})$ :

$$
\begin{aligned}
-B_{1}(u, v)+2 B_{1}\left(D_{y} u, D_{y} v\right)+2 B_{2}\left(\left(D_{y}^{2}+1\right) u,\left(D_{y}^{2}+1\right) v\right) & =\alpha_{0} u \cdot v, \\
-B_{2}(\dot{u}, \dot{v})+2 B_{2}\left(D_{y} \dot{u}, D_{y} \dot{v}\right)+2 B_{1}(\dot{u}, \dot{v}) & =0 .
\end{aligned}
$$

We can use these last two identities regardless of the form of $(u, v)$ and $(\dot{u}, \dot{v})$. Therefore, we formulate them as a system for the symbols of the kernels $B_{1}$ and $B_{2}$ :

$$
\begin{aligned}
(-1+2 \xi \eta) b_{1}+2\left(\xi^{2}+1\right)\left(\eta^{2}+1\right) b_{2} & =-\alpha_{0} \\
(-1+2 \xi \eta) b_{2}+2 b_{1} & =0 .
\end{aligned}
$$

The solution to this system is

$$
b_{1}(\xi, \eta)=\frac{\alpha_{0}(1-2 \xi \eta)}{p(\xi, \eta)}, \quad b_{2}(\xi, \eta)=\frac{2 \alpha_{0}}{p(\xi, \eta)} .
$$

Here the determinantal polynomial $p(\xi, \eta)$ is given by the expression

$$
p(\xi, \eta)=-\left(4 \xi^{2}+4 \eta^{2}-4 \xi \eta+3\right),
$$


which is "elliptic". It is therefore not difficult to see that with this definition, the operators defined on (6.8) obey the bounds (5.5). Moreover, any operators that come up as commutators will satisfy the same estimates.

Lemma 6.2. Let $B_{1}$ and $B_{2}$ be the operators with symbols (6.8) and set

$$
w_{1}=\frac{1}{\rho^{\frac{1}{2}}} B_{1}(u, v)+\frac{1}{\rho^{\frac{1}{2}}} B_{2}(\dot{u}, \dot{v}),
$$

where $\left(\partial_{\rho}^{2}+D_{y}^{2}+1\right) u=F$ and $\left(\partial_{\rho}^{2}+D_{y}^{2}+1\right) v=G$. Then

$$
\mathcal{E}_{\text {quad }}=\left(\partial_{\rho}^{2}+D_{y}^{2}+1\right) w_{1}-\frac{\alpha_{0} u v}{\rho^{\frac{1}{2}}}=\frac{1}{\rho^{1 / 2}} \mathcal{Q}+\mathcal{C}_{1}+\mathcal{C}_{2}+\mathcal{C}_{3},
$$

where

$$
\begin{array}{r}
\mathcal{Q}=B_{1}(F, v)+B_{1}(u, G)-2 B_{2}\left(\left(D_{y}^{2}+1\right) u, G\right)-2 B_{2}\left(F,\left(D_{y}^{2}+1\right) v\right) \\
+B_{2}(\dot{F}, \dot{v})+B_{2}(\dot{u}, \dot{G})+2 B_{2}(F, G) .
\end{array}
$$

To analyze and estimate the commutators we have:

Lemma 6.3. Let $p$ be a symmetric elliptic polynomial of degree two:

$$
p(\xi, \eta)=c_{1}\left(\xi^{2}+\eta^{2}\right)+c_{2} \xi \eta+c_{3}, \quad|p(\xi, \eta)| \geq c(1+|\xi|+|\eta|)^{2}>0 .
$$

We say that $B_{i j}$ is an operator of type $(i, j), 0 \leq i+j \leq 2$, if the kernels satisfy

$$
b_{i j}(\xi, \eta)=\frac{c \xi^{i} \eta^{j}}{p(\xi, \eta)^{k}}
$$

We have

$$
\begin{aligned}
\partial_{\rho}^{k}\left(\frac{1}{\rho^{1 / 2}} B_{i j}(u, v)\right)-\frac{1}{\rho^{1 / 2}} \sum_{\ell=0}^{k}\left(\begin{array}{c}
k \\
\ell
\end{array}\right) B_{i j}\left(\partial_{\rho}^{\ell} u, \partial_{\rho}^{k-\ell} v\right) \\
=\sum_{m=1, \ldots, k} \frac{1}{\rho^{1 / 2+m}} \sum_{\ell=0}^{k-m} B_{i j, m \ell k}\left(\partial_{\rho}^{\ell} u, \partial_{\rho}^{k-m-\ell} v\right)
\end{aligned}
$$

where $B_{i j, m \ell k}$ are operators of the same type as $B_{i j}$.

We say that $B_{1}$ is an operator of type 1 if it is a sum of operators of type $(0,0)$ and $(1,1)$. We say that $B_{2}$ is an operator of type 2 if it is an operator of type $(0,0)$. We say that $B_{4}$, respectively $B_{5}$, is an operator of type 4 if it is an operator of type $(2,0)$, respectively $(0,2)$. We say that $B_{3}$ is an operator of type 3 if it is a sum of operators of type $0,2,4,5$.

If $B_{2}$ is an operator of type 2 , then

$$
B_{2}\left(D_{y}^{2} u, v\right)=B_{4}(u, v), \quad B_{2}\left(u, D_{y}^{2} v\right)=B_{5}(u, v)
$$

where $B_{4}, B_{5}$ are operators of type 4,5 , respectively, and

$$
B_{4}\left(u, D_{y}^{2} v\right)=B_{1}\left(D_{y} u, D_{y} v\right), \quad B_{5}\left(D_{y}^{2} u, v\right)=B_{1}\left(D_{y} u, D_{y} v\right),
$$

where $B_{1}$ is an operator of type 0 . If $B_{1}$ is an operator of type 1 , then

$$
B_{1}\left(D_{y}^{2} u, v\right)=B_{31}\left(D_{y} u, D_{y} v\right)+B_{32}(u, v),
$$

where $B_{3 i}$ are operators of type 3 . Moreover if $B_{2}$ is an operator of type 2 , then

$$
B_{2}\left(D_{y}^{2} u, D_{y}^{2} v\right)=B_{1}\left(D_{y} u, D_{y} v\right)
$$

where $B_{1}$ is an operator of type 1 . 
Proof. According to (5.7) the commutator with $\partial_{\rho}$ is $-\left(\xi \partial_{\xi}+\eta \partial_{\eta}\right) b(\xi, \eta)$. For any homogeneous polynomial of degree two we have $\left(\xi \partial_{\xi}+\eta \partial_{\eta}\right) q(\xi, \eta)=2 q(\xi, \eta)$. The commutator formula follows from this.

Lemma 6.4. With notation as in the previous lemmas we have

$$
\mathcal{C}_{1}=\frac{1}{\rho^{3 / 2}}\left(B_{1,1}(u, \dot{v})+B_{1,1}(\dot{u}, v)\right)+\frac{1}{\rho^{5 / 2}} B_{1,2}(u, v),
$$

$\mathcal{C}_{2}=\frac{1}{\rho^{3 / 2}}\left(B_{4,1}(u, \dot{v})+B_{5,1}(\dot{u}, v)+B_{2,1}(\dot{u}, G-v)+B_{2,1}(F-u, \dot{v})\right)+\frac{1}{\rho^{5 / 2}} B_{2,2}(\dot{u}, \dot{v})$, where we replaced $\ddot{u}=-D_{y}^{2} u-u+F$ and $\ddot{v}=-D_{y}^{2} v-v+G$, and

$$
\mathcal{C}_{3}=\frac{1}{\rho^{3 / 2}}\left(B_{4,2}(u, \dot{v})+B_{5,2}(\dot{u}, v)\right),
$$

where $B_{i, j}$ are operators of type $i$. Moreover

$$
\begin{aligned}
& \partial_{\rho} \mathcal{C}_{1}=\frac{1}{\rho^{3 / 2}}\left(B_{1,2}(\dot{u}, \dot{v})\right.\left.+B_{1,3}(u, v)+B_{3,1}\left(D_{y} u, D_{y} v\right)\right) \\
&+\frac{1}{\rho^{5 / 2}}\left(B_{1,3}(u, \dot{v})+B_{1,3}(\dot{v}, u)\right)+\frac{1}{\rho^{7 / 2}} B_{1,4}(u, v), \\
& \partial_{\rho} \mathcal{C}_{2}=\frac{1}{\rho^{3 / 2}}\left(B_{3,2}(\dot{u}, \dot{v})+B_{3,3}\left(D_{y} u, D_{y} v\right)+B_{4,3}(u, G)+B_{5,3}(F, v)\right) \\
&+\frac{1}{\rho^{3 / 2}}\left(B_{2,3}(F, G)+B_{2,4}(\dot{u}, \dot{G})+B_{2,5}(\dot{F}, \dot{v})\right) \\
&+\frac{1}{\rho^{5 / 2}}\left(B_{3,4}(\dot{u}, v)+B_{3,5}(u, \dot{v})+B_{2,6}(\dot{u}, G)+B_{2,7}(F, \dot{v})\right) \\
&+\frac{1}{\rho^{7 / 2}} B_{2,8}(\dot{u}, \dot{v}),
\end{aligned}
$$

and

$$
\partial_{\rho} \mathcal{C}_{3}=\frac{1}{\rho^{3 / 2}}\left(B_{0,1}\left(D_{y} u, D_{y} v\right)+B_{3,6}(\dot{u}, \dot{v})\right)+\frac{1}{\rho^{5 / 2}}\left(B_{3,7}(u, \dot{v})+B_{3,8}(\dot{u}, v)\right) .
$$

The following two lemmas follow from Proposition 5.2 and Lemma 5.4

Lemma 6.5. For $i=1,2,3$,

(6.26) $\left\|\mathcal{C}_{i}\right\|_{H^{1}} \lesssim \rho^{-3 / 2}\left(\|(u, \dot{u}, F)\|_{H^{1}}\|(v, \dot{v}, G)\|_{B_{\rho}^{\infty}}+\|(u, \dot{u}, F)\|_{B_{\rho}^{\infty}}\|(v, \dot{v}, G)\|_{H^{1}}\right)$,

(6.27) $\left\|\mathcal{C}_{i}\right\|_{B_{\rho}^{\infty}} \lesssim \rho^{-3 / 2}\|(u, \dot{u}, F)\|_{B_{\rho}^{\infty}}\|(v, \dot{v}, G)\|_{B_{\rho}^{\infty}}$,

(6.28) $\left\|\partial_{\rho} \mathcal{C}_{i}\right\|_{H^{1}} \lesssim \rho^{-3 / 2}\left\|\left(u, \dot{u}, D_{y} u, F, \dot{F}\right)\right\|_{H^{1}}\left\|\left(v, \dot{v}, D_{y} v, G, \dot{G}\right)\right\|_{B_{\rho}^{\infty}}$

$$
+\rho^{-3 / 2}\left\|\left(u, \dot{u}, D_{y} u, F, \dot{F}\right)\right\|_{B_{\rho}^{\infty}}\left\|\left(v, \dot{v}, D_{y} v, G, \dot{G}\right)\right\|_{H^{1}},
$$

(6.30) $\left\|\partial_{\rho} \mathcal{C}_{i}\right\|_{B_{\rho}^{\infty}} \lesssim \rho^{-3 / 2}\left\|\left(u, \dot{u}, D_{y} u, F, \dot{F}\right)\right\|_{B_{\rho}^{\infty}}\left\|\left(v, \dot{v}, D_{y} v, G, \dot{G}\right)\right\|_{B_{\rho}^{\infty}}$.

Lemma 6.6. With notation as in the previous lemma we have

$$
\begin{aligned}
\|\mathcal{Q}\|_{B_{\rho}^{\infty}} & \lesssim\|(u, \dot{u}, v, \dot{v}, F, G)\|_{B_{\rho}^{\infty}}\|(F, \dot{F}, G, \dot{G})\|_{B_{\rho}^{\infty}} \\
\|\mathcal{Q}\|_{H^{1}} \lesssim & \|(u, \dot{u}, v, \dot{v}, F, G)\|_{B_{\rho}^{\infty}}\|(F, \dot{F}, G, \dot{G})\|_{H^{1}} \\
& +\|(u, \dot{u}, v, \dot{v}, F, G)\|_{H^{1}}\|(F, \dot{F}, G, \dot{G})\|_{B_{\rho}^{\infty}} .
\end{aligned}
$$


Moreover

$$
\mathcal{Q}=-\frac{\alpha_{0}}{3}(-F v-u G+2 \dot{F} \dot{v}+2 \dot{u} \dot{G}+4 F G)+\mathcal{R},
$$

where for $\sigma<2 / 3$

$$
\left\|P_{\leq \rho^{\sigma}} \mathcal{R}\right\|_{B_{\rho}^{\infty}} \lesssim \rho^{-\sigma}\|(u, \dot{u}, v, \dot{v}, F, G)\|_{B_{\rho}^{\infty} \cap H^{1}}\|(F, \dot{F}, G, \dot{G})\|_{B_{\rho}^{\infty} \cap H^{1}} .
$$

Here $\|u\|_{B_{\rho}^{\infty} \cap H^{1}}=\|u\|_{B_{\rho}^{\infty}}+\|u\|_{H^{1}}$. Furthermore

$$
\begin{aligned}
\left\|\partial_{\rho} \mathcal{Q}\right\|_{B_{\rho}^{\infty}} & \lesssim\|(u, \dot{u}, v, \dot{v}, F, G)\|_{B_{\rho}^{\infty}}\|(F, \dot{F}, L F, G, \dot{G}, L G)\|_{B_{\rho}^{\infty}}, \\
\left\|\partial_{\rho} \mathcal{Q}\right\|_{H^{1}} & \lesssim\|(u, \dot{u}, v, \dot{v}, F, G)\|_{B_{\rho}^{\infty}}\|(F, \dot{F}, L F, G, \dot{G}, L G)\|_{H^{1}} \\
& +\|(u, \dot{u}, v, \dot{v}, F, G)\|_{H^{1}}\|(F, \dot{F}, L F, G, \dot{G}, L G)\|_{B_{\rho}^{\infty}},
\end{aligned}
$$

where $L=\partial_{\rho}^{2}+D_{y}^{2}+1$.

6.2. Estimates for the quadratic terms. We now apply the machinery of the last few sections to the task of estimating the quadratic terms on the RHS of

$$
\left(\partial_{\rho}^{2}-\frac{1}{\rho^{2}} \partial_{y}^{2}+1\right) v=F=\frac{\alpha_{0}}{\rho^{\frac{1}{2}}} v^{2}+\frac{\beta(\rho y)}{\rho} v^{3}+\frac{\beta_{0}}{\rho} v^{3}+\frac{1}{\rho} \mathcal{R}_{\beta}(\rho, y) v^{3} .
$$

To do this, we define the correction:

$$
w_{1}=\frac{1}{\rho^{\frac{1}{2}}} B_{1}(v, v)+\frac{1}{\rho^{\frac{1}{2}}} B_{2}(\dot{v}, \dot{v}),
$$

where $v$ is our solution to $(6.39)$ and $B_{1}$ and $B_{2}$ are the operators with symbols (6.8). We define the associated error as

$$
\mathcal{E}_{\text {quad }}=\left(\partial_{\rho}^{2}+D_{y}^{2}+1\right) w_{1}-\frac{\alpha_{0}}{\rho^{\frac{1}{2}}} v^{2} .
$$

Let us first give a weak decay estimate for $F$ and $L F$.

\section{Lemma 6.7.}

$$
\begin{aligned}
&\|(F, \dot{F})\|_{H^{1}} \lesssim \frac{1}{\rho^{1 / 2}}\left(1+\|(v, \dot{v})\|_{L^{\infty}}\right)\|(v, \dot{v})\|_{L^{\infty}}\|(v, \dot{v})\|_{H^{1}}, \\
&\|(F, \dot{F})\|_{B_{\rho}^{\infty}} \lesssim \frac{1}{\rho^{1 / 2}}\left(1+\|(v, \dot{v})\|_{B_{\rho}^{\infty}}\right)\|(v, \dot{v})\|_{B_{\rho}^{\infty}}^{2},
\end{aligned}
$$

and with $L=\partial_{\rho}^{2}+D_{y}^{2}+1$,

$$
\begin{aligned}
\|L F\|_{H^{1}} & \lesssim \frac{1}{\rho^{1 / 2}}\left(1+\|(v, \dot{v})\|_{L^{\infty}}\right)\|(v, \dot{v})\|_{L^{\infty}}\left(\|(v, \dot{v})\|_{H^{1}}+\|F\|_{H^{1}}\right), \\
\|L F\|_{B_{\rho}^{\infty}} & \lesssim \frac{1}{\rho^{1 / 2}}\left(1+\|(v, \dot{v})\|_{B_{\rho}^{\infty}}\right)\|(v, \dot{v})\|_{B_{\rho}^{\infty}}\left(\|(v, \dot{v})\|_{B_{\rho}^{\infty}}+\|F\|_{B_{\rho}^{\infty}}\right) .
\end{aligned}
$$

Proof. Let us first prove (6.42). First $\rho$ derivatives falling on the coefficients will only improve the decay (for the $\mathcal{R}_{\beta}$ and $\beta$ see Lemma 2.2 and its proof), and $\rho$ derivatives falling on $v$ can be estimated as the terms without the $\rho$ derivative. It therefore only remains to consider the estimate for $F$ itself. If the $y$ derivatives fall on $v$ this is obvious. It therefore only remains to consider the case when the $y$ derivative falls on $\beta$ (or $\mathcal{R}_{\beta}$, which is better behaved by Lemma 2.2). It therefore only remains to estimate

$$
\left\|\beta^{\prime}(\rho y) v^{3}\right\|_{L^{2}} \leq\left\|\beta^{\prime}(\rho y)\right\|_{L^{2}}\left\|v^{3}\right\|_{L^{\infty}} \lesssim \frac{1}{\rho^{1 / 2}}\|v\|_{L^{\infty}}^{3},
$$


where we estimate one factor $\|(v, \dot{v})\|_{L^{\infty}} \leq\|(v, \dot{v})\|_{H^{1}}$. The only terms that could possibly be problematic in proving the estimate (6.44) are when $\partial_{\rho}$ and $D_{y}$ fall on $\beta(\rho y)$. However that just produces terms

$$
\rho^{-1-\ell}(\rho y)^{\ell} \beta^{(k)}(\rho y) v^{3}, \quad \ell \geq 0 .
$$

Since $\left\|(\rho y)^{\ell} \beta^{(k)}(\rho y)\right\|_{H^{1}} \leq C \rho^{1 / 2}$ the estimate follows also for this term.

We now have the following:

\section{Proposition 6.8.}

$$
\left\|\left(w_{1}, \dot{w}_{1}\right)\right\|_{H^{1}} \lesssim \frac{1}{\rho^{1 / 2}}\left(1+\|(v, \dot{v})\|_{L^{\infty}}^{2}\right)\|(v, \dot{v})\|_{B_{\rho}^{\infty}}\|(v, \dot{v})\|_{H^{1}}
$$

$$
\left\|\left(w_{1}, \dot{w}_{1}, D_{y} w_{1}\right)\right\|_{H^{1}} \lesssim \frac{1}{\rho^{1 / 2}}\left(1+\|(v, \dot{v})\|_{L^{\infty}}^{2}\right)\left\|\left(v, \dot{v}, D_{y} v\right)\right\|_{B_{\rho}^{\infty}}\left\|\left(v, \dot{v}, D_{y} v\right)\right\|_{H^{1}},
$$

and

$$
\left\|\left(w_{1}, \dot{w}_{1}, D_{y} w_{1}\right)\right\|_{B_{\rho}^{\infty}} \lesssim \frac{1}{\rho^{1 / 2}}\left(1+\|(v, \dot{v})\|_{B_{\rho}^{\infty}}^{2}\right)\left\|\left(v, \dot{v}, D_{y} v\right)\right\|_{B_{\rho}^{\infty}}^{2}
$$

Proof. The error $\mathcal{E}_{\text {quad }}$ is of the form in Lemma 6.2, and the estimate follows from the estimates for the commutator errors in Lemma 6.5 and the nonlinear errors in Lemma 6.6. Here we estimate one factor $\|(v, \dot{v})\|_{B_{\rho}^{\infty}} \leq C\|(v, \dot{v})\|_{H^{1}}$.

The bilinear estimate in Proposition 5.2 directly gives the estimate for $\left\|w_{1}\right\|_{H^{1}}$. To get the estimate for $\left\|D_{y} w_{1}\right\|_{H^{1}}$ one first applies the commutator in Lemma [5.1. and the term so obtained

$$
\frac{1}{\rho^{1 / 2}} B_{2}\left(\dot{v}, D_{y} \dot{v}\right)
$$

can be estimated as before since the operator $B(u, v)=B_{2}\left(v, D_{y} u\right)$ also satisfies the estimate in Proposition 5.2 To estimate $\left\|\dot{w}_{1}\right\|_{H^{1}}$ we again use the commutator in Lemma 5.1 and obtain the term

$$
\frac{1}{\rho^{1 / 2}} B_{2}(\dot{v}, \ddot{v})
$$

To estimate this term we use the equation $\ddot{v}=D_{y}^{2} v-v+F$. We see that we hence have to control

$$
\frac{1}{\rho^{1 / 2}} B_{2}\left(\dot{v}, D_{y}^{2} v\right)
$$

However $B(u, v)=B_{2}\left(u, D_{y}^{2} v\right)$ is also an operator of the form we have estimates for in Proposition 5.2 
We remark that the above estimates are sufficient for the bootstrap to prove the main theorem in the end of the introduction.

\section{VARIABLE COEFFICIENT CUBIC NORMAL FORMS}

According to the last section we may form the quantity $w=v-w_{1}$, where $w_{1}$ is defined in (6.40), and the equation for $w$ is of the form

$$
\left(\partial_{\rho}^{2}+D_{y}^{2}+1\right) w=\frac{1}{\rho} \beta(\rho y) v^{3}+\frac{\beta_{0}}{\rho} v^{3}-\mathcal{E}_{q u a d}+\mathcal{R},
$$

where $\mathcal{R}$ is given by (1.7) and $\mathcal{E}_{\text {quad }}$ is given by (6.41). To further clean up this expression, we make the following two definitions:

$$
\mathcal{E}_{\text {grand }}=\frac{\beta_{0}}{\rho} v^{3}+\frac{1}{\rho} \beta(\rho y)\left(v^{3}-\left(v-w_{1}\right)^{3}\right)-\mathcal{E}_{\text {quad }}+\mathcal{R} .
$$

With these definitions, we may rewrite (7.1) as

$$
\left(\partial_{\rho}^{2}+D_{y}^{2}+1\right) w=\frac{1}{\rho} \beta(\rho y) w^{3}+\mathcal{E}_{\text {grand }} .
$$

Before continuing on with the analysis of the cubic term on the RHS of this last line, we pause to review the error estimates which we have shown thus far.

\section{Lemma 7.1.}

$$
\begin{aligned}
&\left\|\mathcal{E}_{\text {grand }}\right\|_{H^{1}} \lesssim\left(1+\|(v, \dot{v})\|_{B_{\rho}^{\infty}}^{4}\right) \frac{1}{\rho}\|(v, \dot{v})\|_{B_{\rho}^{\infty}}^{2}\|(v, \dot{v})\|_{H^{1}}, \\
&\left\|\dot{\mathcal{E}}_{\text {grand }}\right\|_{H^{1}} \lesssim\left(1+\|(v, \dot{v})\|_{B_{\rho}^{\infty}}^{6}\right) \frac{1}{\rho}\|(v, \dot{v})\|_{B_{\rho}^{\infty}}^{2}\|(v, \dot{v})\|_{H^{1}}, \\
&\left\|\mathcal{E}_{\text {grand }}\right\|_{B_{\rho}^{\infty}} \lesssim\left(1+\|(v, \dot{v})\|_{B_{\rho}^{\infty}}^{4}\right) \frac{1}{\rho}\|(v, \dot{v})\|_{B_{\rho}^{\infty}}^{3}, \\
&\left\|\dot{\mathcal{E}}_{\text {grand }}\right\|_{B_{\rho}^{\infty}} \lesssim\left(1+\|(v, \dot{v})\|_{B_{\rho}^{\infty}}^{6}\right) \frac{1}{\rho}\|(v, \dot{v})\|_{B_{\rho}^{\infty}}^{3} .
\end{aligned}
$$

Proof. These estimates for $\mathcal{E}_{\text {quad }}$ where previously proven. The only new possibly problematic term is

$$
\frac{1}{\rho} \beta(\rho y)\left(v^{3}-\left(v-w_{1}\right)^{3}\right)=\frac{1}{\rho} \beta(\rho y)\left(3 v^{2}+w_{1}^{2}+3 w_{1} v\right) w_{1},
$$

but the extra $\rho^{-1 / 2}$ decay in $w_{1}$ in the previous section compensates for the loss of $\rho$ when taking the $H^{1}$ norm of $\beta(\rho y)$.

Remark 7.2. We remark that for the constant coefficient cubic $\left(\partial_{\rho}^{2}+D_{y}^{2}+1\right) v=$ $\beta_{0} v^{3} / \rho$ we do not need normal forms to prove global existence; see L-S2. The reason it is needed here is to get an estimate for the $H^{1}$ norm to remove the problematic term that otherwise would be there, $\left(\partial_{y} \beta(\rho y)\right) \rho^{-1} v^{3}=\beta^{\prime}(\rho y) v^{3}$, which does not decay enough in $L^{2}$. These estimates are sufficient for the bootstrap to prove the main theorem in the end of the introduction.

We furthermore note that it is only low frequencies that can cause a problem. 
Lemma 7.3.

$$
\mathcal{E}_{\text {high }}=\frac{1}{\rho} \beta(\rho y)\left(w^{3}-\left(P_{\leq \rho} w\right)^{3}\right)
$$

satisfies

$$
\left\|\mathcal{E}_{h i g h}\right\|_{H^{1}} \lesssim \frac{1}{\rho}\left(1+\|(v, \dot{v})\|_{B_{\rho}^{\infty}}^{4}\right)\|(v, \dot{v})\|_{B_{\rho}^{\infty}}^{2}\|(v, \dot{v})\|_{H^{1}}
$$

Proof. The extra decay comes from the fact that $\left\|P_{\geq \rho} w\right\|_{L^{\infty}} \leq \rho^{-1 / 2}\|w\|_{H^{1}}$, and this decay exactly compensates for the loss of $\rho^{1 / 2}$ when taking the $H^{1}$ norm of $\beta(\rho y)$.

We will therefore attempt to find and subtract off a normal form $w$ such that

$$
\left(\partial_{\rho}^{2}-\frac{1}{\rho^{2}} \partial_{y}^{2}+1\right) w_{2}=\frac{\beta(\rho y)}{\rho}\left(P_{\leq \rho} w\right)^{3}+\mathcal{E}_{c u b i c}
$$

modulo an error $\mathcal{E}_{\text {cubic }}$ that satisfies the estimate in the previous lemma.

In general we decompose $\beta$ into a sum of three functions such that the Fourier transform of the first one vanishes in a neighborhood of the origin and in neighborhoods of $\pm \sqrt{8}$, the support of the Fourier transform of the second one is contained in a small neighborhood of the origin, and the support of the transform of the third one is contained in small neighborhoods of $\pm \sqrt{8}$. The normal form $w_{2}$ above is then obtained by adding up the normal forms obtained for each of the three functions in the decomposition. The case when $\widehat{\beta}(\xi)$ is vanishing in neighborhood of 0 and in neighborhoods of $\pm \sqrt{8}$ was dealt with in the introduction, and the argument in that case is a special case of the argument below, so we will deal with the remaining two cases.

7.1. The case when $\widehat{\beta}(\xi)$ is supported in a neighborhood of 0 . If $\widehat{\beta}$ is supported near 0 , then the $f_{i}$ are actually growing, although the derivatives are bounded. In this case we have to modify the approach, taking into account that the solution decays for large frequencies. Let $f_{i}[\beta], i=0,2$, be the functionals defined by solving the system in the introduction depending on $\beta$; see Definition 1.3. Let us make the frequency decomposition

$$
\beta=\sum_{j=0}^{\infty} \beta_{j}, \quad \text { where } \beta_{j}=P_{2^{-j}} \beta, \quad j \geq 0,
$$

the projection onto a dyadic region of frequencies $\sim 2^{-j}$. We now define

$$
w_{2, j}=\frac{1}{\rho} \sum_{i=0,2} f_{i}\left[\beta_{j}\right] F_{i}\left(w_{j}, \dot{w}_{j}\right), \quad \text { where } \quad w_{j}=P_{\leq \rho / 2^{j}} w, \quad \widehat{\beta}_{j}(\xi)=\chi_{1}\left(2^{j} \xi\right) \widehat{\beta}(\xi) ;
$$

here $\chi_{1}$ is supported in a neighborhood of 1 , and $F_{i}(v, \dot{v})=v^{3-i} \dot{v}^{i}$. Then $w_{2, j}$ solves

$$
\left(\square_{\mathcal{H}}+1\right) w_{2, j}=\rho^{-1} \beta_{j}(\rho y) F_{0}\left(w_{j}\right)+\mathcal{E}_{c u b i c, j} .
$$

We know from the argument in the introduction that for each $j$ the error $\mathcal{E}_{c u b i c, j}$ satisfies the estimate (1.26), but we need to be able to sum up the errors. We will 
sum only over values of $j$ for which $2^{j} \leq \rho^{1 / 2}$ and form

$$
w_{2}=\sum_{j=1}^{\infty} \chi_{0}\left(2^{j} / \rho^{1 / 2}\right) w_{2, j},
$$

where $\chi_{0} \in C_{0}^{\infty}$ is 1 in a neighborhood of the origin. The remainder satisfies our bound (1.26) since

\section{Lemma 7.4.}

$$
\left\|\rho^{-1} P_{\leq \rho^{\sigma}} \beta(\rho y) F_{0}(w)\right\|_{H^{1}} \lesssim \frac{1}{\rho}\|w\|_{L^{\infty}}^{2}\|w\|_{H^{1}}, \quad \sigma \leq 3 / 4 .
$$

Proof. It is easy to see that $P_{\leq \rho^{\sigma}} \beta(\rho y)=\int e^{i y \rho \zeta} \chi\left(\zeta \rho^{1-\sigma}\right) \hat{\beta}(\zeta) d \zeta$ satisfies

$$
\left|\partial_{y}^{k} P_{\leq \rho^{\sigma}} \beta(\rho y)\right| \lesssim \rho^{(k+1) \sigma-1}, \quad\left\|\partial_{y}^{k} P_{\leq \rho^{\sigma}} \beta(\rho y)\right\|_{L_{y}^{2}} \lesssim \rho^{(k+1) \sigma-3 / 2} .
$$

Hence the quantity in the lemma can be bounded by

$\rho^{-1}\left\|P_{\leq \rho^{\sigma}} \beta(\rho y)\right\|_{H^{1}}\left\|F_{0}(w)\right\|_{L^{\infty}}+\rho^{-1}\left\|P_{\leq \rho^{\sigma}} \beta(\rho y)\right\|_{L^{\infty}}\left\|F_{0}(w)\right\|_{H^{1}} \lesssim \rho^{-1}\left\|F_{0}(w)\right\|_{H^{1}}$.

We will show:

Proposition 7.5. Suppose $\widehat{\beta}$ is supported in a neighborhood of the origin. Then with $w_{2}$ as above we have

$$
\left(\square_{\mathcal{H}}+1\right) w_{2}=\rho^{-1} \beta(\rho y) F_{0}(w)+\mathcal{E}_{\text {cubic }},
$$

where for some large $N$

$$
\left\|\mathcal{E}_{\text {cubic }}\right\|_{H^{1}} \lesssim \frac{1}{\rho}\left(1+\|(v, \dot{v})\|_{B_{\rho}^{\infty}}\right)^{N}\|(v, \dot{v})\|_{B_{\rho}^{\infty}}^{2}\|(v, \dot{v})\|_{H^{1}} .
$$

Moreover

$$
\left\|\left(w_{2}, \dot{w}_{2}, D_{y} w_{2}\right)\right\|_{H^{1}} \lesssim \frac{1}{\rho^{1 / 4}}\|(w, \dot{w})\|_{L^{\infty}}^{2}\|(w, \dot{w})\|_{H^{1}} .
$$

What makes this argument work is that $\beta_{j}$ will become small:

\section{Lemma 7.6.}

$$
\left\|\beta_{j}(\rho y)\right\|_{L^{\infty}} \lesssim 1 / 2^{j}, \quad\left\|\beta_{j}(\rho y)\right\|_{\dot{H}^{n}} \lesssim\left(\rho / 2^{j}\right)^{n-1 / 2} / 2^{j}, \quad n=0,1 .
$$

Proof. First by scaling we may assume that $\rho=1$. Since $\widehat{\beta}_{j}(\xi)=\chi_{1}\left(2^{j} \xi\right) \widehat{\beta}(\xi)$ it follows that the $L^{\infty}$ bound follows from

$$
\left\|\beta_{j}\right\|_{L^{\infty}} \lesssim\left\|\widehat{\beta}_{j}\right\|_{L^{1}} \lesssim \int\left|\chi_{1}\left(2^{j} \xi\right)\right| d \xi \lesssim 2^{-j}
$$

and the $L^{2}$ bound from

$$
\left\|\partial_{y}^{n} \beta_{j}\right\|_{L^{2}}^{2} \lesssim\left\|\xi^{n} \widehat{\beta}_{j}\right\|_{L^{2}}^{2} \lesssim \int\left|\xi^{n} \chi_{1}\left(2^{j} \xi\right)\right|^{2} d \xi \lesssim 2^{-j} 2^{-n 2 j}
$$

Therefore the remainder is summable:

\section{Lemma 7.7.}

$$
\left\|\beta_{j}(\rho y) F_{0}\left(w_{j}\right)-\beta_{j}(\rho y) F_{0}(w)\right\|_{\dot{H}^{1}} \lesssim 2^{-j}\|w\|_{\dot{H}^{1}}\|w\|_{L^{\infty}}^{2} .
$$


Proof. This is bounded by

$$
\left\|\beta_{j}(\rho y)\right\|_{\dot{H}^{1}}\left\|F_{0}\left(w_{j}\right)-F_{0}(w)\right\|_{L^{\infty}}+\left\|\beta_{j}(\rho y)\right\|_{L^{\infty}}\left\|F_{0}\left(w_{j}\right)-F_{0}(w)\right\|_{\dot{H}^{1}}
$$

which can be bounded by the previous lemma and

$$
\left\|w-w_{j}\right\|_{L^{\infty}} \lesssim\left(\rho 2^{-j}\right)^{-1 / 2}\|w\|_{\dot{H}^{1}} .
$$

Therefore it only remains to look at the error terms in the approximation (7.14). We first note that it is easy to see that

Lemma 7.8. The support of $\widehat{f_{i}[\beta]}$ is contained in the support of $\widehat{\beta}_{j}$. Moreover, we have

$$
\left\|(\rho y)^{\ell} f_{i}\left[\beta_{j}\right]^{(k)}(\rho y)\right\|_{\dot{H}^{n}} \lesssim\left(\rho 2^{-j}\right)^{n-1 / 2} 2^{-j(k-\ell-1)}
$$

and

$$
\left\|(\rho y)^{\ell} f_{i}\left[\beta_{j}\right]^{(k)}(\rho y)\right\|_{L^{\infty}} \lesssim 2^{-j(k-\ell-1)} .
$$

Proof. First, we note that the $\rho$ factor can be removed by a scaling, so we may assume that $\rho=1$. The functionals $f_{i}\left[\beta_{j}\right]$ are linear combinations of $g_{0}$ and $g_{2}$ satisfying

$$
-\xi^{2} \widehat{g}_{0}(\xi)=-3 \widehat{\beta}_{j}(\xi), \quad\left(-\xi^{2}+8\right) \widehat{g}_{2}(\xi)=-\widehat{\beta}_{j}(\xi),
$$

where $\widehat{\beta}_{j}(\xi)=\chi_{1}\left(2^{j} \xi\right) \widehat{\beta}(\xi)$, and where the support of $\widehat{\beta}$ is bounded away from $\pm \sqrt{8}$. We have

$$
\left\|z^{\ell} g_{0}^{(k)}(z)\right\|_{L^{\infty}} \lesssim\left\|\partial_{\xi}^{\ell} \xi^{k} \widehat{g}_{0}(\xi)\right\|_{L_{\xi}^{1}} \lesssim 2^{j \ell} 2^{-j(k-2)} 2^{-j}
$$

and

$$
\left\|z^{\ell} g_{0}^{(k)}(z)\right\|_{L^{2}} \lesssim\left\|\partial_{\xi}^{\ell} \xi^{k} \widehat{g}_{0}(\xi)\right\|_{L_{\xi}^{2}} \lesssim 2^{j \ell} 2^{-j(k-2)} 2^{-j / 2}
$$

and the estimates for $g_{2}$ are better.

Lemma 7.9. We have

$$
\begin{aligned}
& \text { (7.33) } \frac{1}{\rho}\left(\square_{\mathcal{H}}+1\right) \sum_{i=0,2} f_{i}\left[\beta_{j}\right] F_{i}\left(w_{j}, \dot{w}_{j}\right) \\
& =\frac{1}{\rho} \sum_{i=0,2}\left(\square_{\mathcal{H}}+1\right) f_{i}\left[\beta_{j}\right] F_{i}+2 \partial_{\rho} f_{i}\left[\beta_{j}\right] F_{i}^{1}\left(w_{j}, \dot{w}_{j}\right)+f_{i}\left[\beta_{j}\right] F_{i}^{2}\left(w_{j}, \dot{w}_{j}\right)+\mathcal{E}_{1, j}+\mathcal{E}_{2, j} .
\end{aligned}
$$

Here

$$
\mathcal{E}_{1, j}=\frac{1}{\rho}\left(2 \partial_{\rho} f_{i}\left[\beta_{j}\right]\left(\partial_{\rho} F_{i}-F_{i}^{1}\right)+f_{i}\left[\beta_{j}\right]\left(\partial_{\rho}^{2} F_{i}-F_{i}^{2}\right)\right)
$$

where

$$
\begin{aligned}
& \partial_{\rho} F_{i}-F_{i}^{1}=G_{i}^{1}\left(\ddot{w}_{j}+w_{j}\right), \\
& \partial_{\rho}^{2} F_{i}-F_{i}^{2}=G_{i}^{2}\left(\ddot{w}_{j}+w_{j}\right)+G_{i}^{3}\left(\ddot{w}_{j}+w_{j}\right)^{2}+G_{i}^{4} \partial_{\rho}\left(\ddot{w}_{j}+w_{j}\right),
\end{aligned}
$$

where $G_{i}^{k}=G_{i}^{k}\left(w_{j}, \dot{w}_{j}\right)$ are polynomials such that all terms are cubic, and

$$
\mathcal{E}_{2, j}=\frac{1}{\rho} \sum_{i=0,2} 2 D_{y} f_{i}\left[\beta_{j}\right] D_{y} F_{i}\left(w_{j}, \dot{w}_{j}\right)+f_{i}\left[\beta_{j}\right] D_{y}^{2} F_{i}\left(w_{j}, \dot{w}_{j}\right)
$$


Here we have the following estimates.

Lemma 7.10. We assume as before that $\rho \leq 2^{j}$ :

$$
\left\|\mathcal{E}_{2, j}\right\|_{H^{1}} \lesssim \frac{2^{-j}}{\rho}\left(1+\frac{2^{2 j}}{\rho}+\frac{2^{4 j}}{\rho^{2}}\right)\left\|w_{j}\right\|_{L^{\infty}}^{2}\left\|w_{j}\right\|_{H^{1}} .
$$

Proof. Due to Lemma 7.8, the main term under the sum is the second one. Since $D_{y}=\rho^{-1} \partial_{y}$ is bounded by $2^{j} / \rho$ (in $L^{2}$ ) acting on $w_{j}$, the $H^{1}$ norm of this is bounded by

$$
\sum_{n=0}^{2}\left\|D_{y}^{n} f_{i}\left[\beta_{j}\right]\right\|_{L^{\infty}}\left\|D_{y}^{2-n} F_{i}\right\|_{H^{1}} \lesssim \sum_{n=0}^{2} 2^{-j(n-1)} \frac{2^{j(2-n)}}{\rho^{2-n}}\left\|F_{i}\right\|_{H^{1}} .
$$

Lemma 7.11. If $2^{j} \leq \rho^{1 / 2}$, then for some $N$ :

$$
\left\|\mathcal{E}_{1, j}\right\|_{H^{1}} \lesssim \frac{2^{j / 2}}{\rho^{1 / 2}}\left(1+\|(v, \dot{v})\|_{B_{\rho}^{\infty}}\right)^{N}\|(v, \dot{v})\|_{B_{\rho}^{\infty}}^{2} \frac{1}{\rho}\|(v, \dot{v})\|_{H^{1}} .
$$

Proof. We have

$$
\ddot{w}_{j}+w_{j}=D_{y}^{2} w_{j}+P_{\leq \rho / 2^{j}}\left(\frac{1}{\rho} \beta w^{3}+\mathcal{E}_{\text {grand }}\right)+\left[\partial_{\rho}^{2}, P_{\leq \rho / 2^{j}}\right] w .
$$

Here

$$
\left\|\left[\partial_{\rho}^{2}, P_{\leq \rho / 2^{j}}\right] w\right\|_{H^{1}} \lesssim \frac{1}{\rho}\left\|P_{\sim \rho / 2^{j}} \dot{w}\right\|_{H^{1}}+\frac{1}{\rho^{2}}\left\|P_{\sim \rho / 2^{j}} w\right\|_{H^{1}}
$$

Hence

(7.43) $\left\|\ddot{w}_{j}+w_{j}\right\|_{H^{1}} \lesssim \frac{2^{2 j}}{\rho^{2}}\|w\|_{H^{1}}+\frac{1}{\rho}\|w\|_{L^{\infty}}^{2}\|w\|_{H^{1}}+\frac{1}{\rho}\|(w, \dot{w})\|_{H^{1}}+\left\|\mathcal{E}_{\text {grand }}\right\|_{H^{1}}$.

To estimate the $\rho$ derivative we must use this estimate again since

$$
\left\|\partial_{\rho}\left[\partial_{\rho}^{2}, P_{\leq \rho / 2^{j}}\right] w\right\|_{H^{1}} \lesssim \frac{1}{\rho}\left\|P_{\sim \rho / 2^{j}} \ddot{w}\right\|_{H^{1}}+\frac{1}{\rho^{2}}\left\|P_{\sim \rho / 2^{j}} \dot{w}\right\|_{H^{1}}+\frac{1}{\rho^{3}}\left\|P_{\sim \rho / 2^{j}} w\right\|_{H^{1}} .
$$

Hence if $2^{j} \leq \rho^{1 / 2}$ and $k=0,1$, we get for some $N$ :

$$
\left\|\partial_{\rho}^{k}\left(\ddot{w}_{j}+w_{j}\right)\right\|_{H^{1}} \leq\left(1+\|(v, \dot{v})\|_{B_{\rho}^{\infty}}\right)^{N} \frac{1}{\rho}\|(v, \dot{v})\|_{H^{1}}
$$

Similarly

$$
\left\|\partial_{\rho}^{k}\left(\ddot{w}_{j}+w_{j}\right)\right\|_{B_{\rho}^{\infty}} \leq\left(1+\|(v, \dot{v})\|_{B_{\rho}^{\infty}}\right)^{N} \frac{1}{\rho}\|(v, \dot{v})\|_{B_{\rho}^{\infty}} .
$$

The error terms involving $\rho$ derivatives that we have to bound are the $H^{1}$ norm of

$$
\mathcal{E}_{3, j}=\frac{1}{\rho}\left(\partial_{\rho}^{2} f_{i} F_{i}^{0}+2 \partial_{\rho} f_{i} F_{i}^{1}\right)
$$

plus terms that decay faster in $\rho$.

Lemma 7.12. We have

$$
\left\|\mathcal{E}_{3, j}\right\|_{H^{1}} \lesssim \frac{1}{\rho}\left(2^{j} / \rho\right)^{1 / 2}\left\|\left(w_{j}, \dot{w}_{j}\right)\right\|_{L^{\infty}}^{3}+\left(\left(2^{j} / \rho\right)+2^{-j}\right)\left\|\left(w_{j}, \dot{w}_{j}\right)\right\|_{L^{\infty}}^{2}\|(w, \dot{w})\|_{H^{1}} .
$$


Proof. Taking the $y$ derivative of this we see that we must bound the $L^{2}$ norm of

$$
\rho^{-k}\left(k(\rho y)^{k-1} f_{i}\left[\beta_{j}\right]^{(k)}(\rho y)+(\rho y)^{k} f_{i}\left[\beta_{j}\right]^{(k+1)}(\rho y)\right) F_{i}^{1-k}\left(w_{j}, \dot{w}_{j}\right), \quad k=1,2,
$$

and

$$
\rho^{-1-k}(\rho y)^{k} f_{i}\left[\beta_{j}\right]^{(k)}(\rho y) \partial_{y} F_{i}^{1-k}\left(w_{j}, \dot{w}_{j}\right), \quad k=1,2 .
$$

The error terms involving the $y$ derivatives are

$$
f_{i}\left[\beta_{j}\right]^{(2-k)}(\rho y) \frac{1}{\rho^{1+k}} \partial_{y}^{k} F_{i}\left(w_{j}, \dot{w}_{j}\right), \quad k=1,2,
$$

and if we take the $H^{1}$ norm we see that we must estimate the following terms in $L^{2}$ :

$$
f_{i}\left[\beta_{j}\right]^{(2-k)}(\rho y) \frac{1}{\rho^{1+k}} \partial_{y}^{k+1} F_{i}\left(w_{j}, \dot{w}_{j}\right), \quad k=0,1,2 .
$$

Taking the first factor of (17.49) in $L^{2}$ we get the bound

$$
\rho^{-1}\left(2^{j} / \rho\right)^{1 / 2}\left\|\left(w_{j}, \dot{w}_{j}\right)\right\|_{L^{\infty}}^{3} .
$$

Taking the second factor of (7.50) in $L^{2}$ we get the bound

$$
\rho^{-1}\left(2^{j} / \rho\right)\left\|\left(w_{j}, \dot{w}_{j}\right)\right\|_{L^{\infty}}^{2}\|(w, \dot{w})\|_{H^{1}} .
$$

Taking the second factor of (7.52) in $L^{2}$ we get the bound

$$
\rho^{-1} 2^{-j}\left\|F_{i}\right\|_{\dot{H}^{1}}
$$

since the operator $\partial_{y} / \rho$ is bounded by $2^{-j}$ acting on functions whose frequencies are bounded by $C \rho 2^{-j}$.

The final type of error is when derivatives fall on the factor $\rho^{-1}$ :

$$
\mathcal{E}_{4, j}=\frac{2}{\rho^{2}} \sum_{i=0}^{3}\left(\partial_{\rho} f_{i} F_{i}+f_{i} \partial_{r} F_{i}\right)+\frac{2}{\rho^{3}} \sum_{i=0}^{3} f_{i} F_{i},
$$

which clearly has been controlled by previous arguments. Summing up, we have proven:

Proposition 7.13. We have

$$
\begin{aligned}
\left(\square_{\mathcal{H}}+1\right) & \frac{1}{\rho} \sum_{i=0,2} f_{i}\left[\beta_{j}\right] F_{i}\left(w_{j}, \dot{w}_{j}\right) \\
& =\frac{1}{\rho} \sum_{i=0,2}\left(f_{i}\left[\beta_{j}\right]-f_{i}\left[\beta_{j}\right]^{(2)}\right) F_{i}\left(w_{j}, \dot{w}_{j}\right)+f_{i}\left[\beta_{j}\right] F_{i}^{2}\left(w_{j}, \dot{w}_{j}\right)+\mathcal{E}_{j},
\end{aligned}
$$

where for some $N$,

$$
\sum_{2^{j} \leq \rho^{1 / 2}}\left\|\mathcal{E}_{j}\right\|_{H^{1}} \lesssim \frac{1}{\rho}\left(1+\|(v, \dot{v})\|_{B_{\rho}^{\infty}}\right)^{N}\|(v, \dot{v})\|_{B_{\rho}^{\infty}}^{2}\|(v, \dot{v})\|_{H^{1}}
$$

Since the functionals $f_{i}\left[\beta_{j}\right]$ where chosen so that

$$
\begin{gathered}
\sum_{i=0,2}\left(f_{i}\left[\beta_{j}\right](\rho y)-f_{i}\left[\beta_{j}\right]^{(2)}(\rho y)\right) F_{i}\left(w_{j}, \dot{w}_{j}\right)+f_{i}\left[\beta_{j}\right](\rho y) F_{i}^{2}\left(w_{j}, \dot{w}_{j}\right) \\
=\beta_{j}(\rho y) F_{0}\left(w_{j}, \dot{w}_{j}\right)
\end{gathered}
$$


Proposition 7.5 follows from Proposition 7.13 apart from the estimates for $w_{2}$ itself. For this note that $w_{2}$ has frequencies at most $4 \rho$ so $D_{y}$ is a bounded operator on $w_{2}$.

7.2. The case when $\widehat{\beta}(\xi)$ is supported in a neighborhood of $\pm \sqrt{8}$. If $\widehat{\beta}$ is supported near $\pm \sqrt{8}$, then the best thing we can say is that $f_{0}(\rho y)$ and $f_{2}(\rho y)$ solving the above system are only bounded and more importantly their derivatives no longer decay. Therefore we no longer can assume that their derivatives with respect to $\rho$ are decaying when $|y|$ is bounded from below. In our situation, because $\beta$ is fast decaying, this may be overcome by multiplying by a cutoff $\chi\left(\rho^{a} y\right)$ for some $0<a<1$, where $\chi$ is 1 in a neighborhood of the origin. We will however instead take a different approach and obtain a new more general variable coefficient normalform transformation that is a better approximation as long as $|y|$ is bounded from above. This is obtained by taking into account the $\rho$ derivatives of the system to obtain

$$
\begin{aligned}
\square_{\mathcal{H}} f_{0}-2 f_{0}-2 \partial_{\rho} f_{1}+2 f_{2} & =\beta(\rho y), \\
\square_{\mathcal{H}} f_{1}-6 f_{1}+6 \partial_{\rho} f_{0}-4 \partial_{\rho} f_{2}+6 f_{3} & =0, \\
\square_{\mathcal{H}} f_{2}-6 f_{2}+6 f_{0}+4 \partial_{\rho} f_{1}-6 \partial_{\rho} f_{3} & =0, \\
\square_{\mathcal{H}} f_{3}-2 f_{3}+2 f_{1}+2 \partial_{\rho} f_{2} & =0 .
\end{aligned}
$$

As before, starting with $g_{0}=3 f_{0}+f_{2}, g_{2}=f_{0}-f_{2}, g_{1}=f_{1}+3 f_{3}, g_{3}=f_{1}-f_{3}$, we get

$$
\begin{aligned}
\square_{\mathcal{H}} g_{0}-2 \partial_{\rho} g_{1} & =3 \beta, \\
\square_{\mathcal{H}} g_{1}+2 \partial_{\rho} g_{0} & =0, \\
\square_{\mathcal{H}} g_{2}-8 g_{2}-6 \partial_{\rho} g_{3} & =\beta, \\
\square_{\mathcal{H}} g_{3}-8 g_{3}+6 \partial_{\rho} g_{2} & =0 .
\end{aligned}
$$

Complexifying the above system we get that

$$
K_{1}=\left(g_{0}+i g_{1}\right) e^{i \rho} / 3 \quad \text { and } \quad K_{3}=\left(g_{2}+i g_{3}\right) e^{3 i \rho}
$$

give the system

$$
\begin{array}{lrl}
\left(\square_{\mathcal{H}}+1\right) K_{1}=e^{i \rho} \beta(\rho y) & (0 \text { resonance equation }), \\
\left(\square_{\mathcal{H}}+1\right) K_{3}=e^{3 i \rho} \beta(\rho y) & ( \pm \sqrt{8} \text { resonance equation }) .
\end{array}
$$

We note that we only have to solve these equations asymptotically, which can be done with the stationary phase method. We hence want an asymptotic solution $K_{n}[\beta]$ that solves

$$
\left(\square_{\mathcal{H}}+1\right) K_{n}=e^{i n \rho} \beta(x)+\mathcal{E}_{K_{n}}
$$

where the error $\mathcal{E}_{n}[\beta]$ decays sufficiently fast. Here the functional $K_{n}[\beta]$ and error $\mathcal{E}_{n}[\beta]$ satisfy the same kinds of estimates we had for the functionals $f_{i}[\beta]$ before; i.e. if $\beta$ is smooth and fast decaying we have

$$
\begin{aligned}
\left|\partial_{\rho}^{k} D_{y}^{l} \chi K_{i}\right| & \leqslant C_{k, l}, \quad\left|\partial_{\rho}^{k} D_{y}^{l} \chi \mathcal{E}_{K_{i}}\right| \leqslant \rho^{-1} C_{k, l}, \\
\|\left(\chi K_{i}, D_{y} \chi K_{i}, \partial_{\rho} \chi K_{i} \|_{B_{\rho}^{\infty}}\right. & \lesssim 1 .
\end{aligned}
$$

Here $\chi=\chi(y)$ is a smooth bump function in the $y$ variable. This will be proven in the next section. Assuming that this is true we now define $g_{i}[\beta]$ by (7.58) and $f_{i}[\beta]$ 
from $g_{i}[\beta]$. In that way we get approximate solutions

$$
\begin{aligned}
\square_{\mathcal{H}} g_{0}-2 \partial_{\rho} g_{1} & =3 \beta+\mathcal{E}_{g, 0}, \\
\square_{\mathcal{H}} g_{1}+2 \partial_{\rho} g_{0} & =\mathcal{E}_{g, 1}, \\
\square_{\mathcal{H}} g_{2}-8 g_{2}-6 \partial_{\rho} g_{3} & =\beta+\mathcal{E}_{g, 2}, \\
\square_{\mathcal{H}} g_{3}-8 g_{3}+6 \partial_{\rho} g_{2} & =\mathcal{E}_{g, 3},
\end{aligned}
$$

and

$$
\begin{aligned}
\square_{\mathcal{H}} f_{0}-2 f_{0}-2 \partial_{\rho} f_{1}+2 f_{2} & =\beta(\rho y)+\mathcal{E}_{f, 0}, \\
\square_{\mathcal{H}} f_{1}-6 f_{1}+6 \partial_{\rho} f_{0}-4 \partial_{\rho} f_{2}+6 f_{3} & =\mathcal{E}_{f, 1}, \\
\square_{\mathcal{H}} f_{2}-6 f_{2}+6 f_{0}+4 \partial_{\rho} f_{1}-6 \partial_{\rho} f_{3} & =\mathcal{E}_{f, 2}, \\
\square_{\mathcal{H}} f_{3}-2 f_{3}+2 f_{1}+2 \partial_{\rho} f_{2} & =\mathcal{E}_{f, 3},
\end{aligned}
$$

where $\mathcal{E}_{g, i}$ and $\mathcal{E}_{f, i}$ satisfy the same kinds of estimates as $\mathcal{E}_{K_{i}}$. However since we assume that $\widehat{\beta}$ vanishes in a neighborhood of the origin, $g_{0}[\beta]$ and $g_{1}[\beta]$ can be defined as before by $\widehat{g}_{0}(\xi)=\widehat{\beta}(\xi) / \xi^{2}$ and $\widehat{g}_{1}=0$.

With $f_{i}$ defined as above we see that the only additional error we have to bound in $H^{1}$ is

$$
\frac{1}{\rho}\left\|\sum_{i=0}^{3} \mathcal{E}_{f, i} F_{i}\right\|_{H^{1}} \lesssim \frac{1}{\rho} \sum_{i=0}^{3}\left\|\mathcal{E}_{f, i}\right\|_{C^{1}}\left\|F_{i}\right\|_{H^{1}} \lesssim \frac{1}{\rho} \sum_{i=0}^{3}\left\|F_{i}\right\|_{H^{1}} \lesssim\|(w, \dot{w})\|_{L^{\infty}}^{2}\|(w, \dot{w})\|_{H^{1}} .
$$

\section{A RESONANT PARAMETRICS CONSTRUCtion}

We need to asymptotically solve the complex equations

$$
\begin{array}{lrl}
\left(\square_{\mathcal{H}}+1\right) K_{1} \sim e^{i \rho} \beta(\rho y) & (0 \text { resonance equation }), \\
\left(\square_{\mathcal{H}}+1\right) K_{3} \sim e^{3 i \rho} \beta(\rho y) & ( \pm \sqrt{8} \text { resonance equation }) .
\end{array}
$$

The reader should keep in mind that this system is a more detailed replacement for the system above, when the derivative with respect to $\rho$ was removed. It will allow us to precisely track the dispersive behavior of the $\pm \sqrt{8}$ resonances.

We will only need to solve the system (8.1) - (8.2) asymptotically. To do this, we now compute an approximate fundamental solution to the equation $\square_{\mathcal{H}}+1=$ $\partial_{\rho}^{2}-\rho^{-2} \partial_{y}^{2}+1$ which becomes more and more accurate as $\rho \rightarrow \infty$. First, we define the approximate phase:

$$
\psi(\rho, s ; \xi)=\int_{s}^{\rho}\left(\frac{1}{\zeta^{2}} \xi^{2}+1\right)^{\frac{1}{2}} d \zeta,
$$

and then use this to define the integral kernel:

$$
U(\rho, s ; \xi)=\frac{\sin (\psi(\rho, s ; \xi))}{\left(\frac{1}{s^{2}} \xi^{2}+1\right)^{\frac{1}{2}}} .
$$

Finally, for a source term $H(\rho, y)$ we form the approximate Duhamel integral:

$$
\begin{aligned}
u(\rho, y) & =\frac{1}{2 \pi} \int_{\rho_{0}}^{\rho} U(\rho, s ; \xi) e^{i y \xi} \widehat{H}(s, \xi) d \xi d s \\
& =\int_{\rho_{0}}^{\rho} U\left(\rho, s ; \partial_{y}\right) H(s, y) d s,
\end{aligned}
$$


where $\widehat{H}$ is the Fourier transform (3.1) of $H$ with respect to $y$. This kernel attempts to construct a solution to $\left(\square_{\mathcal{H}}+1\right) u=H$ with vanishing Cauchy data when $\rho=\rho_{0}$. However, this solution is not exact as can easily be seen. We will estimate the error terms shortly.

We now define the normal-form coefficients $K_{i}$ using the formula (8.4):

$$
\begin{aligned}
& K_{1}(\rho, y)=\int_{1}^{\rho} e^{i s} U\left(\rho, s ; \partial_{y}\right) \beta \chi_{1}\left(\frac{s}{\rho}\right) d s, \\
& K_{3}(\rho, y)=\int_{1}^{\rho} e^{3 i s} U\left(\rho, s ; \partial_{y}\right) \beta \chi_{1}\left(\frac{s}{\rho}\right) d s .
\end{aligned}
$$

Here $\chi_{1}$ is a smooth bump function with $\chi_{1}(\zeta) \equiv 1$ when $\frac{1}{2}<|\zeta|<2$, and vanishing close to $\zeta=0$. With these definitions, we form the error terms:

$$
\left(\square_{\mathcal{H}}+1\right) K_{1}-e^{i \rho} \beta(\rho y)=\mathcal{E}_{K_{1}}, \quad\left(\square_{\mathcal{H}}+1\right) K_{3}-e^{3 i \rho} \beta(\rho y)=\mathcal{E}_{K_{3}} .
$$

The estimates we will need are the following.

Proposition 8.1 (Estimates for medium frequency cubic NF coefficients). Consider the functions $K_{1}, K_{3}$, and $\mathcal{E}_{K_{1}}, \mathcal{E}_{K_{3}}$ defined above, where $\beta$ is a Schwartz class function with $\widetilde{\beta}(\xi) \equiv 0$ for $|\xi| \ll 1$ and $1 \ll|\xi|$. Then the following pointwise estimates hold:

$$
\begin{aligned}
\left|\partial_{\rho}^{k} D_{y}^{l} \chi K_{i}\right| & \leqslant C_{k, l}, \quad\left|\partial_{\rho}^{k} D_{y}^{l} \chi \mathcal{E}_{K_{i}}\right| \leqslant \rho^{-1} C_{k, l}, \\
\|\left(\chi K_{i}, D_{y} \chi K_{i}, \partial_{\rho} \chi K_{i} \|_{B_{\rho}^{\infty}}\right. & \lesssim 1 .
\end{aligned}
$$

Here $\chi=\chi(y)$ is a smooth bump function in the $y$ variable.

These estimates will in turn easily follow from:

Lemma 8.2 (Stationary phase estimates). Consider the oscillatory integrals

$$
I_{k}^{ \pm}(\rho, y)=\rho^{-1} \int_{0}^{\rho} \int_{\mathbb{R}} e^{ \pm i \psi(\rho, s ; \xi)} e^{i y \xi} e^{i k s} \mathfrak{m}(\rho, s ; \xi) \chi_{1}\left(\frac{s}{\rho}\right) d \xi d s
$$

where $\mathfrak{m}(\rho, s ; \xi)$ is a symbol such that $\mathfrak{m}(\rho, s ; \xi) \equiv 0$ for $s^{-1}|\xi| \ll 1$ and $1 \ll s^{-1}|\xi|$, and such that

$$
\left|\partial_{s}^{i} \partial_{\rho}^{j} \partial_{\xi}^{l} \mathfrak{m}\right| \leqslant \rho^{-i-j-l} C_{i, j, l},
$$

when $s \sim \rho$. The phase $\psi$ is from (8.3), and the cutoff $\chi_{1}$ is defined as in the previous paragraph. Then for $k=1,3$ the following uniform estimate holds:

$$
\left|\chi(y) I_{k}^{ \pm}(\rho, y)\right| \lesssim 1
$$

Proof of estimate (8.12). We will treat the two cases $k=1$ and $k=3$ separately. In the case where $k=1$, the total phase is nonstationary on the $\xi$ support of $\mathfrak{m}(\rho, s ; \xi)$. We have that

$$
\partial_{s}[ \pm \psi(\rho, s ; \xi)+s]=\mp\left(\frac{1}{s^{2}} \xi^{2}+1\right)^{\frac{1}{2}}+1 \sim 1,
$$

as long as $\frac{1}{s^{2}} \xi^{2} \sim 1$, which holds owing to the support properties of $\mathfrak{m}(\rho, s ; \xi)$ we are assuming. Therefore, integrating by parts one time with respect to $s$ and then using the symbol bounds (8.11) and the $\xi$ support properties of $\mathfrak{m}(\rho, s ; \xi)$, we easily have (8.12) in the case $k=1$.

We now turn to the case $k=3$, which is the main work in establishing estimate (8.12). Here the total phase always has a stationary point, so we need to use 
stationary phase techniques to evaluate the integral. For the most part this turns out to be standard, although a bit of care is needed to deal with the temporal boundary $s=\rho$. We remark here that it is likely the entire estimate can be done in a very general context by considering Gaussian integrals on half spaces, but we will avoid generalities of this form and simply work directly with the specific form of our phase when the stationary point is sufficiently close to the temporal boundary. As a preliminary reduction, we only consider the case of the integral $I^{+}$restricted to the branch of $\mathfrak{m}(\rho, s ; \xi)$ where $\xi>0$. Other combinations are similar and left to the reader.

Under the restriction just imposed, we have the total phase:

$$
\Phi(\rho, y ; s, \xi)=\psi(\rho, s ; \xi)+3 s+y \xi .
$$

First note that on the range where $s \sim \xi \sim \rho$, and $|y| \lesssim 1$, this phase obeys uniform derivative bounds of the form

$$
\left|\partial_{s}^{i} \partial_{\xi}^{j} \Phi\right| \lesssim(s+|\xi|)^{1-i-j} .
$$

The gradient of this phase where $\xi>0$ has components:

$$
\partial_{s} \Phi=-\left(\frac{1}{s^{2}} \xi^{2}+1\right)^{\frac{1}{2}}+3, \quad \partial_{\xi} \Phi=\int_{s / \xi}^{\rho / \xi} \frac{1}{\sqrt{1+\zeta^{2}}} \frac{d \zeta}{\zeta}+y .
$$

Clearly, for fixed values of $\rho$ and $y$ with $|y| \leqslant 1$, there is a unique stationary point $\left(s_{0}, \xi_{0}\right)$ in the range of our integrations. At this (or any) point, the phase Hessian has components:

$$
\begin{aligned}
\partial_{s}^{2} \Phi & =\frac{\xi^{2}}{s^{2}} \frac{1}{\sqrt{\xi^{2}+s^{2}}}, \\
\partial_{s} \partial_{\xi} \Phi & =-\frac{\xi}{s} \frac{1}{\sqrt{\xi^{2}+s^{2}}}, \\
\partial_{\xi}^{2} \Phi & =\frac{1}{\sqrt{\xi^{2}+s^{2}}}-\frac{1}{\sqrt{\xi^{2}+\rho^{2}}} .
\end{aligned}
$$

The phase determinant and trace are thus computed to be

$$
\begin{aligned}
& D=\partial_{s}^{2} \Phi \cdot \partial_{\xi}^{2} \Phi-\left(\partial_{s} \partial_{\xi} \Phi\right)^{2}=-\frac{\xi^{2}}{s^{2}} \frac{1}{\sqrt{\xi^{2}+s^{2}}} \frac{1}{\sqrt{\xi^{2}+s^{2}}} \\
& T=\partial_{s}^{2} \Phi+\partial_{\xi}^{2} \Phi=\frac{\sqrt{\xi^{2}+s^{2}}}{s^{2}}-\frac{1}{\sqrt{\xi^{2}+\rho^{2}}} .
\end{aligned}
$$

Therefore, in the region where $s \sim \rho \sim \xi$, which is where our integrand is restricted, we have both $|D| \sim \rho^{-2}$ and $|T| \lesssim \rho^{-1}$. Therefore, we easily have that the eigenvalues of the phase Hessian in this region are

$$
\lambda_{1}\left(s_{0}, \xi_{0}\right) \sim \rho^{-1}, \quad \lambda_{2}\left(s_{0}, \xi_{0}\right) \sim-\rho^{-1} .
$$

This is the correct balance of factors needed to prove (8.12). Again, the main nonstandard issue is to deal with the integration boundary where $s=\rho$. To handle this, we first decompose the integral (8.10) into two bulk pieces $I_{3}^{+}=I^{\text {near }}+I^{\text {far }}$ 
where

$$
\begin{aligned}
I^{\text {near }} & =\rho^{-1} \int_{0}^{\rho} \int_{\mathbb{R}} e^{i \Phi} \mathfrak{m} \chi\left(\rho^{-\frac{1}{2}}(\rho-s)\right) \chi_{1}\left(\frac{s}{\rho}\right) d \xi d s, \\
I^{\text {far }} & =\rho^{-1} \int_{0}^{\rho} \int_{\mathbb{R}} e^{i \Phi} \mathfrak{m}\left[1-\chi\left(\rho^{-\frac{1}{2}}(\rho-s)\right)\right] \chi_{1}\left(\frac{s}{\rho}\right) d \xi d s .
\end{aligned}
$$

Here $\chi$ is a $C_{0}^{\infty}$ function with $\chi \equiv 1$ in a neighborhood of the origin.

The estimate (8.12) for $I^{\text {far }}$ is completely standard. From the uniform bounds (8.13), we may assume without loss of generality 1 that $\mathfrak{m}(\rho, s ; \xi)$ is supported in a region where there is a global change of variables $(s, \xi)=F(\tau, \eta)$ with uniform derivative bounds:

$$
\left|\partial_{\tau}^{i} \partial_{\eta}^{j} F\right| \leqslant C_{i, j}(1+|\tau|+|\eta|)^{1-i-j},
$$

and such that (notice that from (8.15) the critical point $\left(s_{0}, \xi_{0}\right)$ is hyperbolic):

$$
\Phi \circ F(\tau, \eta)=\rho^{-1}\left(\tau^{2}-\eta^{2}\right)+\Phi\left(s_{0}, \xi_{0}\right) .
$$

In fact, we have by Taylor's formula with integral remainder $\Phi(s, \xi)=\Phi\left(s_{0}, \xi_{0}\right)+\left(s-s_{0}\right)^{2} \Phi_{11}(s, \xi)+\left(s-s_{0}\right)\left(\xi-\xi_{0}\right) \Phi_{12}(s, \xi)+\left(\xi-\xi_{0}\right)^{2} \Phi_{22}(s, \xi)$, where

$$
\Phi_{i j}(s, \xi)=\int_{0}^{1}(1-t)\left(\partial_{i} \partial_{j} \Phi\right)\left(\left(s_{0}, \xi_{0}\right)+t\left(s-s_{0}, \xi-\xi_{0}\right)\right) d t .
$$

Since we have uniform bounds (independent of $\rho$ and $y$ with the above restrictions)

$$
\left|\partial_{s}^{k} \partial_{\xi}^{l} \Phi_{i j}(s, \xi)\right| \lesssim \rho^{-1}(1+s+|\xi|)^{-k+l}, \quad \Phi_{11}(s, \xi) \sim \rho^{-1}, \quad D(s, \xi) \sim \rho^{-2},
$$

the uniform bound for the change of variables obtained by completing the square as in the usual proof of the Morse lemma follows. Therefore, after the further change of variables $\underline{\tau}=\rho^{-\frac{1}{2}} \tau$ and $\underline{\eta}=\rho^{-\frac{1}{2}} \eta$, we may write the integral (8.17) as follows:

$$
I^{f a r}=e^{i \Phi\left(s_{0}, \xi_{0}\right)} \iint e^{i\left(\underline{\tau}^{2}-\underline{\eta}^{2}\right)} \mathfrak{n}(\underline{\tau}, \underline{\eta}) d \underline{\tau} d \underline{\eta},
$$

where $\mathfrak{n}$ is some new symbol obeying the bounds $\left|\partial_{\underline{\tau}}^{i} \partial_{\underline{\eta}}^{j} \mathfrak{n}\right| \leqslant C_{i, j}$, and compactly supported in some large ball (of radius $\sim \rho^{\frac{1}{2}}$, although the size does not matter). The bound (8.12) for integrals of this form is a simple matter of integration by parts away from where $|\underline{\tau}| \leqslant 1$ and $|\eta| \leqslant 1$.

It remains to deal with the integral (8.16). There are two cases here depending on the size of the spatial variable $y$. In the easy case, where $\rho^{-\frac{1}{2}} \ll|y|$, the phase $\Phi$ is nonstationary on the support of the integrand. This is easily confirmed from the second of the gradient calculations (8.14), which in the region where $\rho-s \lesssim \rho^{\frac{1}{2}}$ may be written as

$$
\partial_{\xi} \Phi=\frac{\rho-s}{\xi} h(\rho, s ; \xi)+y \sim y,
$$

as long as $\rho^{-\frac{1}{2}} \leqslant C^{-1}|y|$ for a sufficiently large constant $C$, which depends only on how we cut out the integration region of $I^{\text {near }}$ to begin with. Here $h$ is a function obeying the uniform derivative bounds:

$$
\left|\partial_{s}^{i} \partial_{\xi}^{j} h\right| \leqslant C_{i, j} \rho^{-i-j}
$$

\footnotetext{
${ }^{1}$ This may be done by considering a sufficiently small $O(\rho)$ ball about the stationary point $\left(s_{0} \xi_{0}\right)$. In the compliment of this region the phase $\Phi$ is nonstationary, so a simple integration by parts argument suffices to produce (8.12).
} 
Thus, under the assumption that $\rho^{-\frac{1}{2}} \ll|y|$ we have in the region where $\rho-s \lesssim \rho^{\frac{1}{2}}$ and $\rho \sim \xi$ the symbol bound:

$$
\left|\left(\partial_{\xi} \frac{1}{\partial_{\xi} \Phi}\right) \mathfrak{m}\right| \leqslant C \rho^{-\frac{1}{2}} .
$$

The bound (8.12) easily follows from this and one integration by parts with respect to $\xi$.

One final task here is to establish (8.12) for the integral (8.16) under the additional assumption that $|y| \lesssim \rho^{-\frac{1}{2}}$. In this case, the $O\left(\rho^{\frac{1}{2}}\right)$ stationary region around the point $\left(s_{0}, \xi_{0}\right)$ contains the boundary $s=\rho$, and a bit of care is needed to achieve the desired result. The main difficulty is the following: for very small values of $|y|$ the boundary phase $\left.\Phi\right|_{s=\rho}$ can be quite degenerate due to the hyperbolic nature of the critical point $\left(s_{0}, \xi_{0}\right)$. In fact, if $y=0$, then $\left.\partial_{\xi} \Phi\right|_{s=\rho}=0$. This means that simply integrating by parts with respect to the characteristic directions of the eigenvalues from (8.15) can leave one with a singular boundary term that does not oscillate enough to recover uniform boundedness. The way to get around this is to carefully delineate a new stationary region where one cannot control the phase and then integrate by parts on the compliment. Our first decomposition is to cut $I^{\text {near }}=I_{1}^{\text {near }}+I_{2}^{\text {near }}$ where

$$
\begin{aligned}
I_{1}^{\text {near }} & =\rho^{-1} \int_{0}^{\rho} \int_{\mathbb{R}} \mathcal{I}^{\text {near }} \chi\left(C \rho^{-1}\left(\rho^{-1}+|y|\right)^{-1}(\rho-s)\right) d \xi d s, \\
I_{2}^{\text {near }} & =\rho^{-1} \int_{0}^{\rho} \int_{\mathbb{R}} \mathcal{I}^{\text {near }}\left[1-\chi\left(C \rho^{-1}\left(\rho^{-1}+|y|\right)^{-1}(\rho-s)\right)\right] d \xi d s .
\end{aligned}
$$

Here $\mathcal{I}^{\text {near }}$ is the integrand of the original $I^{\text {near }}$, and $C$ is a flexible large constant. For $C$ sufficiently large, the second integral $I_{2}^{\text {near }}$ above is estimated directly via integration by parts with respect to $\xi$. On the support of the corresponding cutoff, a short calculation shows that one has access to the symbol bounds:

$$
\left|\left(\partial_{\xi} \frac{1}{\partial_{\xi} \Phi}\right)^{i} \mathfrak{m}\right| \leqslant C_{i}(1+\rho-s)^{-i} .
$$

The bound (8.12) for $I_{2}^{\text {near }}$ is therefore a result of two integrations by parts with respect to the $\xi$ variable and then directly estimating the absolute value of the resulting integral.

We now move on to estimating the integral $I_{1}^{\text {near }}$ defined in the last paragraph. This may be further decomposed as $I_{1}^{\text {near }}=I_{1,1}^{\text {near }}+I_{1,2}^{\text {near }}$ where

$$
\begin{aligned}
& I_{1,1}^{\text {near }}=\rho^{-1} \int_{0}^{\rho} \int_{\mathbb{R}} \mathcal{I}_{1}^{\text {near }} \chi\left(C^{-2}\left(\rho^{-1}+|y|\right)(\xi-\sqrt{8} \rho)\right) d \xi d s, \\
& I_{1,2}^{\text {near }}=\rho^{-1} \int_{0}^{\rho} \int_{\mathbb{R}} \mathcal{I}_{1}^{\text {near }}\left[1-\chi\left(C^{-2}\left(\rho^{-1}+|y|\right)(\xi-\sqrt{8} \rho)\right)\right] d \xi d s .
\end{aligned}
$$

Here $C$ is the same large constant used above, which again is assumed to be sufficiently large. This time $\mathcal{I}_{1}^{\text {near }}$ is the integrand of the original $I_{1}^{\text {near }}$. Notice that the support of the integrand in $I_{1,1}^{\text {near }}$ is a rectangle of dimensions $\sim$ $\rho^{-1}\left(\rho^{-1}+|y|\right)^{-1} \times\left(\rho^{-1}+|y|\right)$. Therefore, the bound (8.12) for the integral $I_{1,1}^{\text {near }}$ follows from direct absolute integration.

Our last task is to estimate the integral $I_{1,2}^{\text {near }}$ via another integration by parts argument. Notice that it suffices to consider only the case where $\rho^{-1} \leqslant|y|$, since in the complimentary case the integrand of $I_{1,2}^{\text {near }}$ is supported on a slab where $\rho-s \lesssim 1$, 
and the bound (8.12) in this case again follows from direct absolute integration. This time, we first integrate by parts once with respect to the $s$ variable, which gives us

$$
\begin{aligned}
I_{1,2}^{\text {near }}=-\rho^{-1} \int_{0}^{\rho} \int_{\mathbb{R}} e^{i \Phi} \partial_{s}[\mathfrak{n}(\rho, s ; \xi) \chi & \left.\left(C(\rho|y|)^{-1}(\rho-s)\right)\right] d \xi d s \\
& +\rho^{-1} e^{3 i \rho} \int_{\mathbb{R}} e^{i y \xi} \mathfrak{n}(\rho, \rho ; \xi) d \xi
\end{aligned}
$$

where $\mathfrak{n}$ is the truncated symbol:

$$
\mathfrak{n}(\rho, s ; \xi)=\frac{1}{i \partial_{s} \Phi(\rho, s ; \xi)} \mathfrak{m}(\rho, s ; \xi)\left[1-\chi\left(C^{-2}|y|(\xi-\sqrt{8} \rho)\right)\right]
$$

A few quick calculations show that for this symbol, we have the following derivative bounds as long as we choose $C$ sufficiently large:

$$
\begin{aligned}
\left|\partial_{s} \mathfrak{n}(\rho, s ; \xi)\right| & \lesssim \frac{\rho}{(\xi-\sqrt{8} \rho)^{2}}\left[1-\chi\left(C^{-2}|y|(\xi-\sqrt{8} \rho)\right)\right], \\
\left|\partial_{\xi} \mathfrak{n}(\rho, \rho ; \xi)\right| & \lesssim \frac{\rho}{(\xi-\sqrt{8} \rho)^{2}}\left[1-\chi\left(C^{\prime-2}|y|(\xi-\sqrt{8} \rho)\right)\right]
\end{aligned}
$$

when $\rho-s \lesssim \rho|y|$ and $|y| \lesssim \rho^{-1 / 2}$. Moreover, since $\rho-s \lesssim \rho|y|$ :

$$
\left|\left(\partial_{\xi} \frac{1}{\partial_{\xi} \Phi}\right)^{i}(\mathfrak{n}) \cdot(\rho|y|)^{-1} \chi^{\prime}\left(C(\rho|y|)^{-1}(\rho-s)\right)\right| \leqslant C_{i}(1+\rho-s)^{-i} .
$$

Therefore, either by directly integrating or, if necessary, integrating by parts with respect to $\xi$ one or two times, we can easily see that we have the bound (8.12) for all terms on the RHS of (8.18). For the first term on the RHS of (8.18) we use both (8.19) and (8.21) which allows us to estimate

$$
\begin{aligned}
& \left|\rho^{-1} \int_{0}^{\rho} \int_{\mathbb{R}} e^{i \Phi} \partial_{s}\left[\mathfrak{n}(\rho, s ; \xi) \chi\left(C(\rho|y|)^{-1}(\rho-s)\right)\right] d \xi d s\right| \\
\lesssim & \int_{0}^{\rho} \int_{\mathbb{R}} \frac{1}{(\xi-\sqrt{8} \rho)^{2}}\left[1-\chi\left(C^{-2}|y|(\xi-\sqrt{8} \rho)\right)\right] \chi\left(C(\rho|y|)^{-1}(\rho-s)\right) d \xi d s \\
& +\rho^{-1} \int_{0}^{\rho} \int_{|\xi| \lesssim \rho}(1+\rho-s)^{-2} d \xi d s \\
\lesssim & \rho|y|^{2}+1 .
\end{aligned}
$$

Using the condition that $|y| \lesssim \rho^{-\frac{1}{2}}$, this last formula yields the desired bound. Finally, the last term on the RHS of (8.18) is estimated in a similar fashion via (8.20) and one integration by parts with respect to $\xi$ :

$$
\begin{aligned}
\left|\rho^{-1} \int_{\mathbb{R}} e^{i y \xi} \mathfrak{n}(\rho, \rho ; \xi) d \xi\right| & \lesssim \int_{\mathbb{R}} \frac{|y|^{-1}}{(\xi-\sqrt{8} \rho)^{2}}\left[1-\chi\left(C^{-2}|y|(\xi-\sqrt{8} \rho)\right)\right] d \xi \\
& \lesssim 1 .
\end{aligned}
$$

This completes our demonstration of estimate (8.12).

Proof of the estimates (8.8)- (8.9). We begin with a preliminary reduction, which is that it suffices to consider the first set of estimates (8.8). More specifically, we claim that the first estimate in (8.8) implies the estimate in (8.9). To see this, simply note that the frequency of $\beta(s \rho)$ in both of the integrals (8.5) to the region where $|\xi| \sim \rho$ owing to the presence of the cutoff $\chi_{1}\left(\frac{s}{\rho}\right)$ and the fact 
that $\beta(s \rho)$ is assumed to have frequency support where $|\xi| \sim s$. Therefore, the bulk of the frequency of $\chi K_{i}$ is contained where $|\xi| \sim \rho$ modulo a piece with very fast decay in $|\xi|$ as $\rho \ll|\xi| \rightarrow \infty$ due to the Schwartz tails of $\widehat{\chi}(\xi)$.

We now prove the first estimate in (8.8). Because we have $\left|D_{y}^{k} \chi\right| \leqslant C_{k}$ and $\partial_{\rho} \chi=0$, it suffices to prove that $\left|\chi \partial_{\rho}^{k} D_{y}^{l} K_{i}\right| \leqslant C_{k, l}$. This follows immediately from the estimate (8.12) if we can show that $\partial_{\rho}^{k} D_{y}^{l} K_{i}$ has a symbol $\mathfrak{m}_{k, l}$ obeying (8.11). By decomposing (8.5)-(8.6) into exponentials, it suffices to consider the integrals:

$$
\begin{aligned}
& I_{1}^{ \pm}=\rho^{-1} \int_{\rho_{0}}^{\rho} \int_{\mathbb{R}} e^{ \pm i \psi(\rho, s ; \xi)} e^{i y \xi} e^{i s} \frac{\rho s^{-1}}{\sqrt{s^{-2} \xi^{2}+1}} \widetilde{\beta}\left(s^{-1} \xi\right) \chi_{1}\left(\frac{s}{\rho}\right) d \xi d s, \\
& I_{3}^{ \pm}=\rho^{-1} \int_{\rho_{0}}^{\rho} \int_{\mathbb{R}} e^{ \pm i \psi(\rho, s ; \xi)} e^{i y \xi} e^{3 i s} \frac{\rho s^{-1}}{\sqrt{s^{-2} \xi^{2}+1}} \widetilde{\beta}\left(s^{-1} \xi\right) \chi_{1}\left(\frac{s}{\rho}\right) d \xi d s .
\end{aligned}
$$

Here $\widetilde{\beta}$ is the Fourier transform of $\beta(z)$. Notice that $\frac{\rho s^{-1}}{\sqrt{s^{-2} \xi^{2}+1}} \widetilde{\beta}\left(s^{-1} \xi\right) \chi_{1}\left(\frac{s}{\rho}\right)$ obeys the symbol bound (8.11), and the space of all functions of course forms an algebra. Thus, we only need to show that the $D_{y}$ and $\partial_{\rho}$ derivatives of the phase $\pm \psi+y \xi$ obeys (8.11) on the region where $s \sim \rho \sim \xi$ as well. This easily follows from the definition of (8.3).

The proof of the second bound on (8.8) is very similar. The only difference here is that one needs to first compute the expression $\left(\square_{\mathcal{H}}+1\right) K_{i}$ and compare it to the RHS of (8.1) - 8.2), and then show that the resulting difference is an integral with a symbol $\mathfrak{n}$ such that the quantity $\mathfrak{m}=\rho^{2} \mathfrak{n}$ obeys the bounds (8.11) (further derivatives of the error are handled similarly). We deal only with the expression for $K_{1}$, because the corresponding calculations for $K_{3}$ are completely analogous. A short calculation reveals that

$$
\begin{gathered}
\left(\square_{\mathcal{H}}+1\right) K_{1}-e^{i \rho} \beta(\rho y)=\int_{1}^{\rho}\left(\square_{\mathcal{H}}+1\right)\left[U\left(\rho, s ; \partial_{y}\right) \beta \chi_{1}\left(\frac{s}{\rho}\right)\right] d s \\
=-\frac{1}{\rho} \int_{1}^{\rho} \int_{\mathbb{R}} \cos (\psi(\rho, s ; \xi)) e^{i y \xi} e^{i s} \frac{\xi^{2}}{\rho^{2}} \frac{1}{\sqrt{\xi^{2}+\rho^{2}}} \frac{\rho s^{-1}}{\sqrt{s^{-2} \xi^{2}+1}} \widetilde{\beta}\left(s^{-1} \xi\right) \chi_{1}\left(\frac{s}{\rho}\right) d \xi d s \\
-\frac{2}{\rho} \int_{1}^{\rho} \int_{\mathbb{R}} \cos (\psi(\rho, s ; \xi)) e^{i y \xi} e^{i s} \frac{1}{\sqrt{\rho^{-2} \xi^{2}+1}} \frac{\rho s^{-1}}{\sqrt{s^{-2} \xi^{2}+1}} \widetilde{\beta}\left(s^{-1} \xi\right) \chi_{1}^{\prime}\left(\frac{s}{\rho}\right)\left(\frac{s}{\rho^{2}}\right) d \xi d s \\
\quad+\frac{1}{\rho} \int_{1}^{\rho} \int_{\mathbb{R}} \sin (\psi(\rho, s ; \xi)) e^{i y \xi} e^{i s} \frac{\rho s^{-1}}{\sqrt{s^{-2} \xi^{2}+1}} \widetilde{\beta}\left(s^{-1} \xi\right) \partial_{\rho}^{2}\left[\chi_{1}\left(\frac{s}{\rho}\right)\right] d \xi d s .
\end{gathered}
$$

The reader may easily verify that the symbol in each integral on the right hand side of this last equation obeys the bounds (8.11) with an extra factor of $\rho^{-1}$ to spare. Therefore, after an application of (8.12) we have the desired bounds.

\section{ACKNOWLEDGMENTS}

This work began as a joint project with I. Rodnianski and J. Sterbenz. The authors thank both for their major contributions. The reader is referred to $\mathrm{St}$ for a concise version of the proof based on a preliminary version of this work.

\section{REFERENCES}

[D1] Jean-Marc Delort, Existence globale et comportement asymptotique pour l'équation de Klein-Gordon quasi linéaire à données petites en dimension 1 (French, with English and French summaries), Ann. Sci. École Norm. Sup. (4) 34 (2001), no. 1, 1-61, DOI 10.1016/S0012-9593(00)01059-4. MR1833089(2002h:35194) 
[D2] Jean-Marc Delort, Correction to "Existence globale et comportement asymptotique pour l'équation de Klein-Gordon quasi linéaire à données petites en dimension 1". Ann. Sci. École Norm. Sup. (4) 39 (2006), no. 2, 335-345. MR:2245535 (2007c:35109)

[F] M. Flato, J.C.H. Simon, and E. Taflin, The Maxwell-Dirac equations: the Cauchy problem, asymptotic completeness and the infrared problem, Memoirs of the AMS 128, No. 606 (1997) ( $\mathrm{x}+312$ pages $)$

[H-Nau] Nakao Hayashi and Pavel I. Naumkin, Asymptotics for large time of solutions to the nonlinear Schrödinger and Hartree equations, Amer. J. Math. 120 (1998), no. 2, 369389. MR1613646 (99f:35190)

[H1] Lars Hörmander, Lectures on nonlinear hyperbolic differential equations, Mathématiques \& Applications (Berlin) [Mathematics \& Applications], vol. 26, Springer-Verlag, Berlin, 1997. MR 1466700 (98e:35103)

[H2] Lars Hörmander, The lifespan of classical solutions of nonlinear hyperbolic equations, Pseudodifferential operators (Oberwolfach, 1986), Lecture Notes in Math., vol. 1256, Springer, Berlin, 1987, pp. 214-280, DOI 10.1007/BFb0077745. MR897781 (88j:35024)

[Kat] Jun Kato and Fabio Pusateri, A new proof of long-range scattering for critical nonlinear Schrödinger equations, Differential Integral Equations 24 (2011), no. 9-10, 923-940. MR.2850346

[K1] Sergiu Klainerman, Global existence of small amplitude solutions to nonlinear KleinGordon equations in four space-time dimensions, Comm. Pure Appl. Math. 38 (1985), no. 5, 631-641, DOI 10.1002/cpa.3160380512. MR803252(87e:35080)

[L1] Hans Lindblad, On the lifespan of solutions of nonlinear wave equations with small initial data, Comm. Pure Appl. Math. 43 (1990), no. 4, 445-472, DOI 10.1002/cpa.3160430403. MR 1047332 (91i:35129)

[L2] Hans Lindblad, Global solutions of nonlinear wave equations, Comm. Pure Appl. Math. 45 (1992), no. 9, 1063-1096, DOI 10.1002/cpa.3160450902. MR1177476 (94a:35080)

[L-R] Hans Lindblad and Igor Rodnianski, The weak null condition for Einstein's equations (English, with English and French summaries), C. R. Math. Acad. Sci. Paris 336 (2003), no. 11, 901-906, DOI 10.1016/S1631-073X(03)00231-0. MR1994592 (2004h:83008)

[L-S1] Hans Lindblad and Avy Soffer, A remark on long range scattering for the nonlinear Klein-Gordon equation, J. Hyperbolic Differ. Equ. 2 (2005), no. 1, 77-89, DOI 10.1142/S0219891605000385. MR2134954 (2006d:35143)

[L-S2] Hans Lindblad and Avy Soffer, A remark on asymptotic completeness for the critical nonlinear Klein-Gordon equation, Lett. Math. Phys. 73 (2005), no. 3, 249-258, DOI 10.1007/s11005-005-0021-y. MR2188297(2006i:35249)

[M-S] Nicholas Manton and Paul Sutcliffe, Topological solitons, Cambridge Monographs on Mathematical Physics, Cambridge University Press, Cambridge, 2004. MR2068924 (2006d:58020)

[Sh] Jalal Shatah, Normal forms and quadratic nonlinear Klein-Gordon equations, Comm. Pure Appl. Math. 38 (1985), no. 5, 685-696, DOI 10.1002/cpa.3160380516. MR803256 (87b:35160)

[St] J. Sterbenz, Dispersive decay for the $1 D$ Klein-Gordon equation with variable coefficient nonlinearities, preprint, http://arxiv.org/abs/1307.4808 (2013).

[S-Taf] Jacques C. H. Simon and Erik Taflin, The Cauchy problem for nonlinear Klein-Gordon equations, Comm. Math. Phys. 152 (1993), no. 3, 433-478. MR1213298 (94d:35110)

[Str] Walter A. Strauss, Dispersion of low-energy waves for two conservative equations, Arch. Rational Mech. Anal. 55 (1974), 86-92. MR0352743 (50 \#5230)

Department of Mathematics, Johns Hopkins University, 404 Krieger Hall, 3400 N. Charles Street, Baltimore, Maryland 21218

E-mail address: lindblad@math.jhu.edu

Department of Mathematics, Rutgers University, 110 Frelinghuysen Road, PiscatAWAY, NEW JERSEY 08854

E-mail address: soffer@math.rutgers.edu 